University of South Florida

DIGITAL COMMONS Digital Commons @ University of @ UNIVERSITY OF SOUTH FLORIDA South Florida

\title{
Dynamic Delivery of the National Transit Database Sampling
}

Manual

CUTR

Follow this and additional works at: https://digitalcommons.usf.edu/cutr_nctr

\section{Recommended Citation}

"Dynamic Delivery of the National Transit Database Sampling Manual," National Center for Transit Research (NCTR) Report No. CUTR-NCTR-RR-2011-04, Center for Urban Transportation Research, University of South Florida, 2013.

DOI: https://doi.org/10.5038/CUTR-NCTR-RR-2011-04

Available at: https://scholarcommons.usf.edu/cutr_nctr/109

This Technical Report is brought to you for free and open access by the National Center for Transit Research (NCTR) Archive (2000-2020) at Digital Commons @ University of South Florida. It has been accepted for inclusion in Research Reports by an authorized administrator of Digital Commons @ University of South Florida. For more information, please contact digitalcommons@usf.edu. 


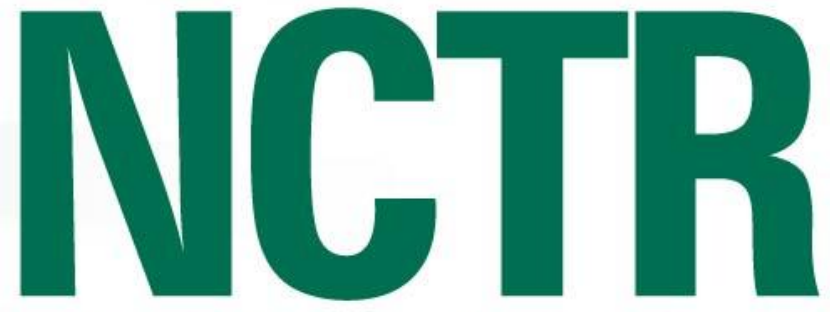

NATIONAL CENTER for TRANSIT RESEARCH

Final Report

\section{Dynamic Delivery of the National Transit Database Sampling Manual}

February 2013

PREPARED FOR

Florida Department of Transportation BDK85 977-28

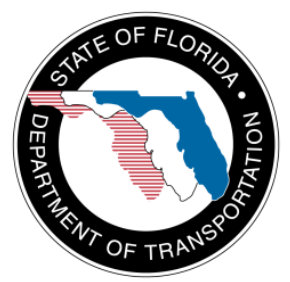




\title{
Dynamic Delivery of the National Transit Database Sampling Manual
}

\author{
FDOT BDK85 977-28
}

Prepared for:

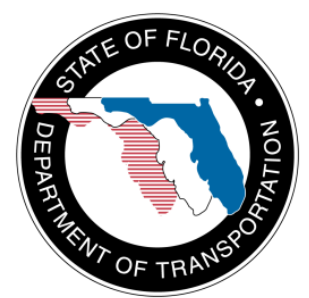

Florida Department of Transportation Diane Quigley, Project Manager

Prepared by:

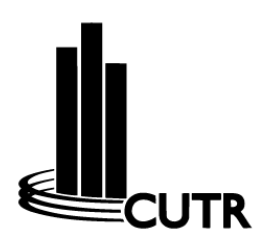

USF Center for Urban Transportation Research

Xuehao Chu, Senior Research Associate

Final Report

February 2013 


\section{Disclaimer}

The opinions, findings, and conclusions expressed in this publication are those of the authors and not necessarily those of the State of Florida Department of Transportation. 


\section{Metric Conversion}

\begin{tabular}{|c|c|c|c|c|}
\hline SYMBOL & WHEN YOU KNOW & MULTIPLY BY & TO FIND & SYMBOL \\
\hline \multicolumn{5}{|c|}{ LENGTH } \\
\hline in & inches & 25.4 & millimeters & $\mathrm{mm}$ \\
\hline $\mathbf{f t}$ & feet & 0.305 & meters & $\mathrm{m}$ \\
\hline yd & yards & 0.914 & meters & $\mathrm{m}$ \\
\hline mi & miles & 1.61 & kilometers & $\mathrm{km}$ \\
\hline \multicolumn{5}{|c|}{ VOLUME } \\
\hline fl oz & fluid ounces & 29.57 & milliliters & $\mathrm{mL}$ \\
\hline gal & gallons & 3.785 & liters & L \\
\hline $\mathrm{ft}^{3}$ & cubic feet & 0.028 & cubic meters & $\mathrm{m}^{3}$ \\
\hline$y d^{3}$ & cubic yards & 0.765 & cubic meters & $\mathrm{m}^{3}$ \\
\hline \multicolumn{5}{|c|}{ NOTE: volumes greater than $1000 \mathrm{~L}$ shall be shown in $\mathrm{m}^{3}$} \\
\hline \multicolumn{5}{|c|}{ MASS } \\
\hline $\mathbf{O z}$ & ounces & 28.35 & grams & $\mathrm{g}$ \\
\hline lb & pounds & 0.454 & kilograms & $\mathrm{kg}$ \\
\hline $\mathbf{T}$ & short tons $(2000 \mathrm{lb})$ & 0.907 & $\begin{array}{c}\text { megagrams } \\
\text { (or "metric ton") }\end{array}$ & Mg (or "t") \\
\hline \multicolumn{5}{|c|}{ TEMPERATURE (exact degrees) } \\
\hline${ }^{\circ} \mathbf{F}$ & Fahrenheit & $\begin{array}{c}5(F-32) / 9 \\
\text { or }(F-32) / 1.8\end{array}$ & Celsius & ${ }^{\circ} \mathrm{C}$ \\
\hline
\end{tabular}




\section{Technical Report Documentation}

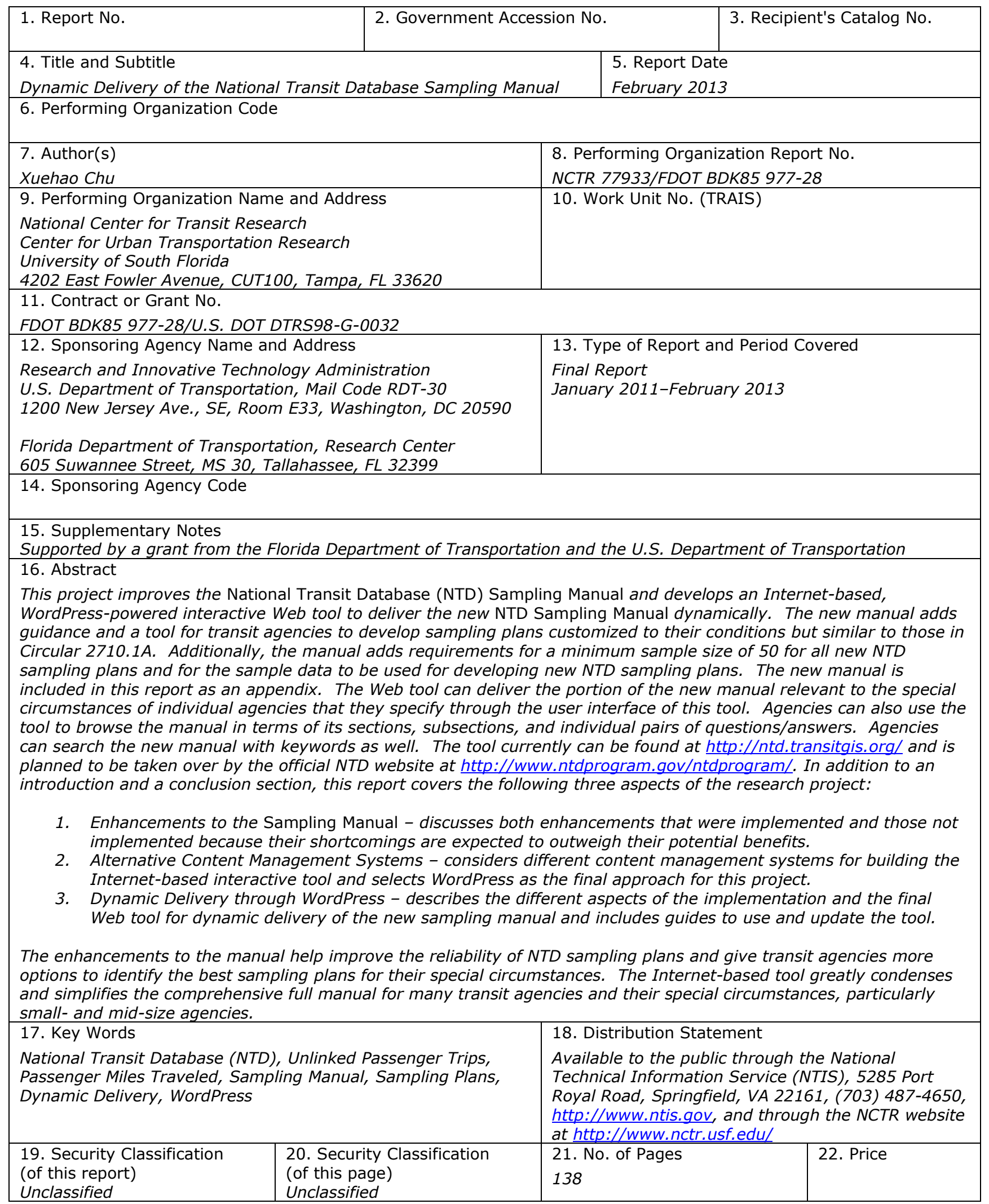




\section{Acknowledgments}

John Giorgis and Sergio Maia of the Federal Transit Administration (FTA) provided insight into how some of the suggested enhancements to the existing NTD Sampling Manual could best be implemented. Special thanks to the GIS Program at the Center for Urban Transportation Research (CUTR), particularly Martin Catalá and Donald Hayward, who researched alternative content management systems for building the Internet-based interactive Web tool to dynamically deliver the NTD Sampling Manual and selected and implemented WordPress for this project. 


\section{Executive Summary}

\section{Background}

In Spring 2010, the Federal Transit Administration (FTA) officially released the National Transit Database (NTD) Sampling Manual, which was developed by the National Center for Transit Research (NCTR) and sponsored by the Florida Department of Transportation (FDOT) in response to an FTA request. The manual consists of guidance for individual transit agencies to get sampling plans, collect sample data, and estimate annual totals of unlinked passenger trips and passenger miles traveled that meet FTA requirements. For three decades, FTA has provided guidance for transit agencies in Circulars 2710.1A and 2710.2A. However, these current circulars cover only two modes-bus and demand response; they do not reflect the operating conditions of individual agencies, and they do not take advantage of modern sampling techniques. The NTD Sampling Manual overcomes the shortcomings of the current circulars and is expected to reduce reporting burdens significantly for a wide range of transit agencies in Florida and across the nation. The experiences since the Spring 2010 release have been mostly positive and confirm this expectation.

The current manual was designed to be comprehensive to cover all modes, service types, units of measurement, methods of sampling and estimation, steps of data collection and estimation, etc. Being comprehensive, however, also comes with a price-only a small portion of the guidance is directly relevant to any one specific application. Some guidance is common to all applications, but other guidance is unique to one or more applications. Because this manual is in the traditional static format, it is not practical to separate the guidance for all possible applications. As a result, agencies need to look for the relevant guidance for each application in this format. This is common to the traditional model of static content delivery, which pushes technical documents that cover many options and variations to an audience with varying needs.

\section{Objectives}

The primary objective of this project was to move the NTD Sampling Manual from its current static delivery format to a dynamic delivery format. This was accomplished by developing an Internet-based interactive tool where the full content of the manual is stored in terms of individual topics, but only the relevant topics are dynamically assembled and presented to a user once the user specifies the profile of a specific application.

Another important objective of this project was to make enhancements to the current version of the NTD Sampling Manual. The experiences since the Spring 2010 release of the current version and changes in FTA's reporting requirements for NTD also prompted the desire to improve the manual in the following ways:

- Ensure that the sampling plans developed from the NTD Sampling Template are based on robust and precise sample data

- Provide more options to transit agencies in developing sampling plans 
- $\quad$ Further reduce the reporting burden to transit agencies, particularly for small- and medium-size agencies

- Simplify procedures that are relatively complex.

\section{Findings and Conclusions}

This project successfully revised the NTD Sampling Manual to incorporate a number of enhancements to the existing version. To ensure quality data for developing sampling plans, for example, the new manual requires a minimum sample size of 50 for all new sampling plans and for all sample data to be used in developing new sampling plans. To give transit agencies more options to seek the best sampling plans for them, as another example, the new manual provides guidance and a tool for transit agencies to develop sampling plans that are customized to their conditions and have a structure similar to those in Circular 2710.1A. Furthermore, the new manual simplifies guidance for transit agencies to determine whether they should revise their previously-developed sampling plans.

Researchers on this project also investigated several potential enhancements and ultimately decided not to incorporate them into the new manual. These potential enhancements had been suggested by FTA to reduce the likely negative impact on transit agencies as a result of the recent split in NTD of the traditional single mode of bus services into three modesregular bus, commuter bus, and bus rapid transit. One suggestion was to allow combined sampling across these three bus modes. Another was to waive the newly-added minimum sample size of 50 for certain services.

Researchers also successfully developed a Web tool for the new NTD Sampling Manual to be delivered to its users in a range of options. Users can download the entire manual and the Sampling Template. In addition, users can browse the manual by its major sections, subsections, or even individual pairs of questions/answers. Users also can specify what they need in terms of four profiling questions (e.g., What is your mode of service?) and their various options (e.g., vanpool), and the Web tool, in turn, can deliver the relevant content of the manual. The tool currently is located at http://ntd.transitgis.org/ and will be taken over by the NTD website at http://www.ntdprogram.gov/ntdprogram/.

However, the new Web tool does not always dynamically deliver contents that are relevant only to the profile specified. Some questions cover guidance for one mode of service in one part of the answer but for another mode of service in other parts of the answer. These pairs of questions/answers were not restructured in the new version to avoid having too many questions and to avoid appearing significantly different from its existing version. This shortcoming also brings forth a lesson for future efforts of developing Web-based dynamic content delivery tools-the full content document should be prepared from the very beginning in such a way that each of its individual topics does not include content for more than one option of any given profiling question. 


\section{Benefits}

The enchancements to the NTD Sampling Manual help improve the reliability of NTD sampling plans and the resulting rideship data reported and give transit agencies more options to search for the best sampling plans for their special circumstances. The Web tool greatly condenses and simplifies the comprehensive full manual for many transit agencies and their special circumstances, particularly small- and mid-size agencies. 


\section{Table of Contents}

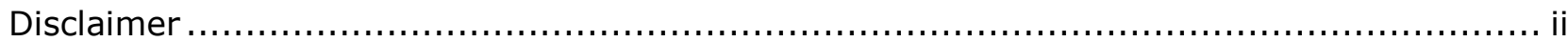

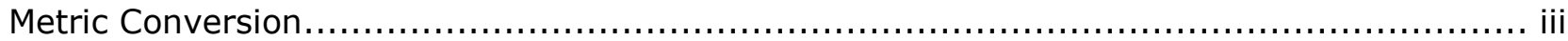

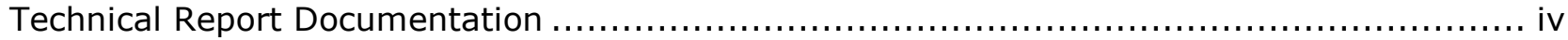

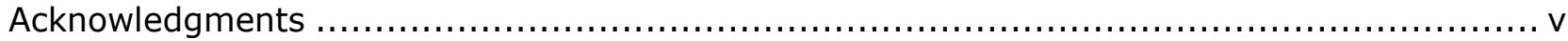

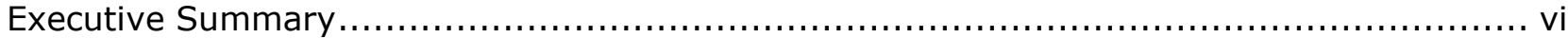

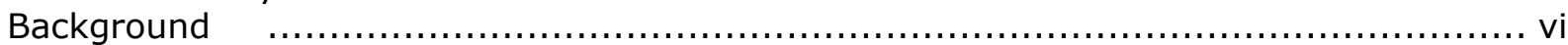

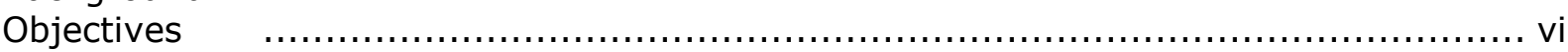

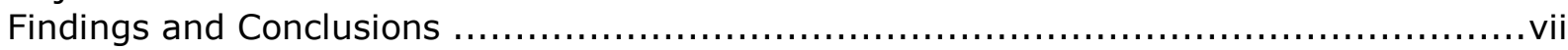

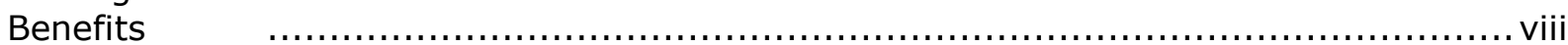

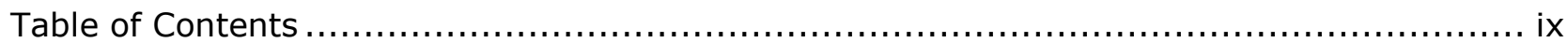

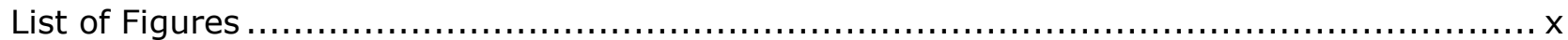

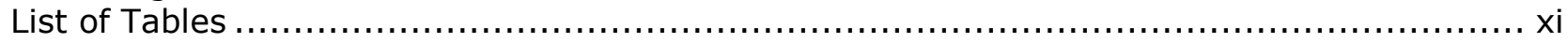

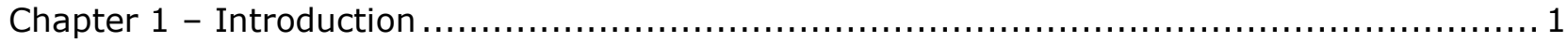

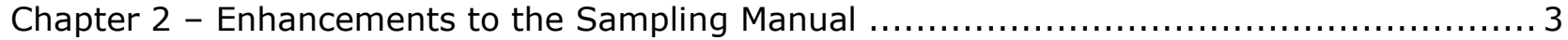

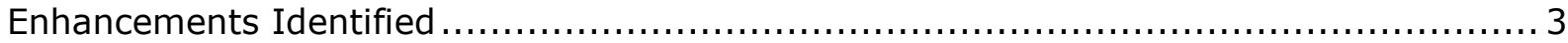

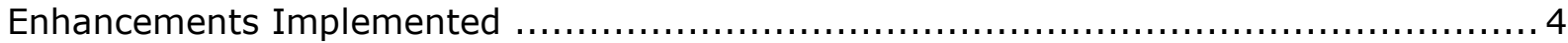

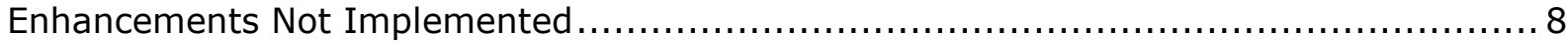

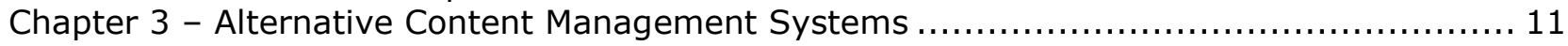

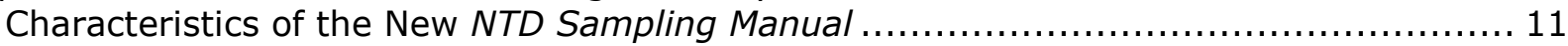

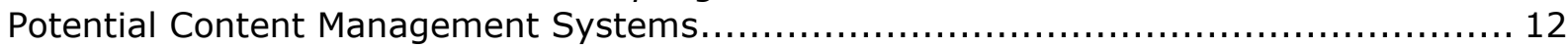

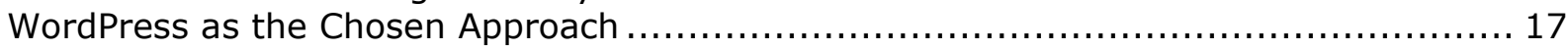

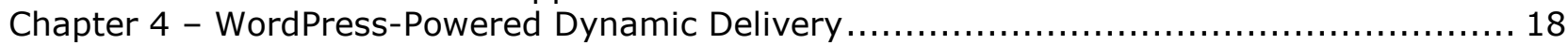

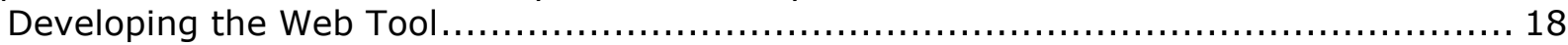

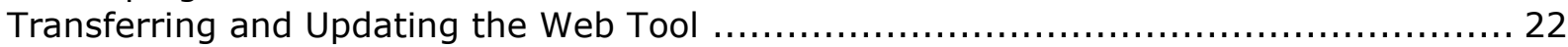

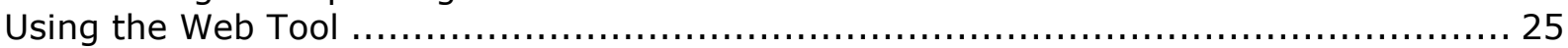

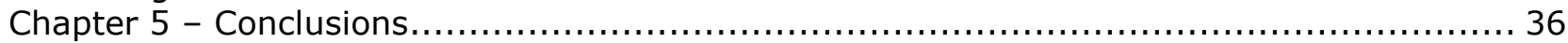

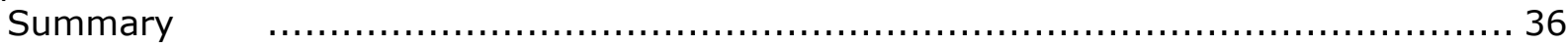

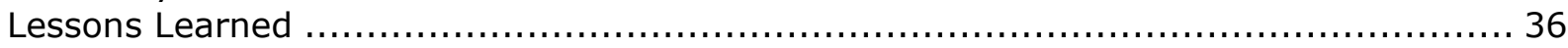

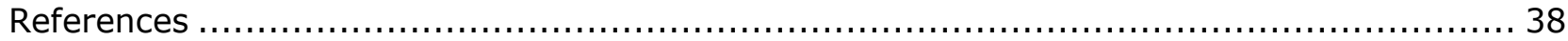

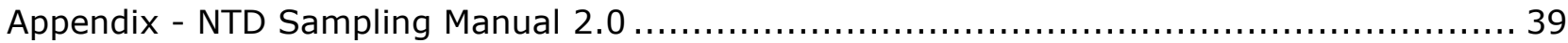




\section{List of Figures}

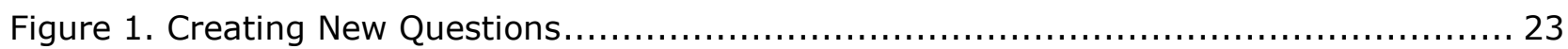

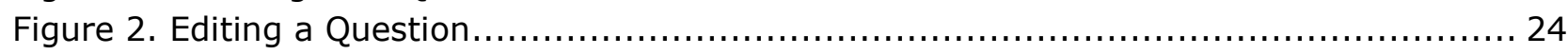

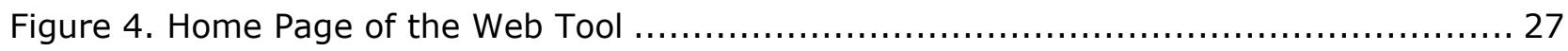

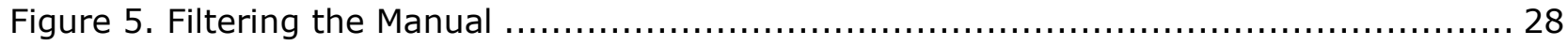

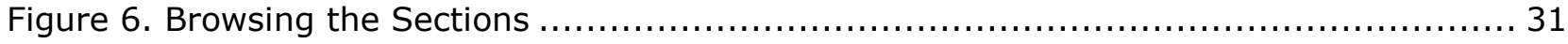

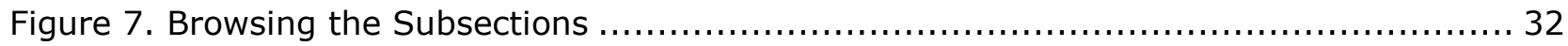

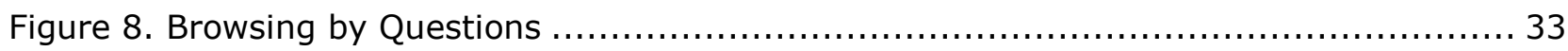

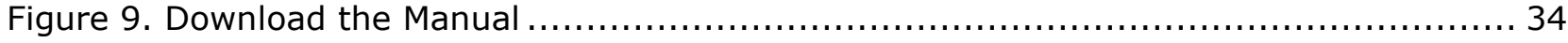

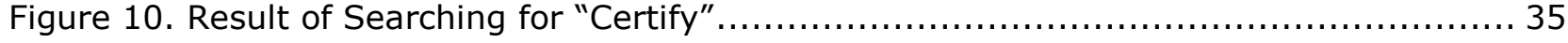




\section{List of Tables}

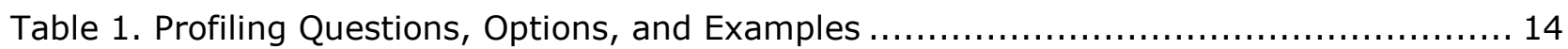

Table 2. Link between Profiling Manual Content.......................................... 20

Table 3. Profiling Questions and Profiling Options for the User Interface ...................... 29

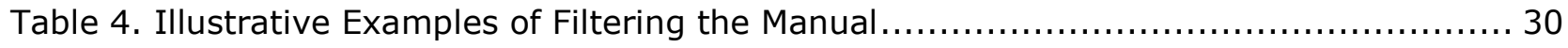




\section{Chapter 1 - Introduction}

In Spring 2010, the Federal Transit Administration (FTA) officially released the first version of the National Transit Database (NTD) Sampling Manual, which was developed by the National Center for Transit Research (NCTR) in response to an FTA request. This manual consists of guidance for individual transit agencies to get sampling plans, collect sample data, and estimate annual total ridership that meets FTA's statistical requirements. For three decades, FTA has provided guidance for transit agencies in Circulars 2710.1A and 2710.2A. But these current circulars cover only two modes-bus and demand response; they do not reflect the operating conditions of individual agencies, and they do not take advantage of modern sampling techniques. The NTD Sampling Manual overcomes the shortcomings of the current circulars and is expected to reduce reporting burdens significantly for a wide range of transit agencies. User experiences since the Spring 2010 release have been mostly positive and confirm this expectation. To serve transit agencies even better, however, FTA has identified from these experiences a number of enhancements to the first version and to the delivery of the manual.

The NTD Sampling Manual was designed to be comprehensive to cover all modes, service types, units of measurement, methods of sampling and estimation, steps of data collection and estimation, etc. Being comprehensive, however, also comes with a price-only a small portion of the guidance is directly relevant to any one specific application. Some guidance is common to all applications, but other guidance is unique to one or more applications. Because the current manual is in the traditional static document-based format, it is not practical to separate the guidance for all possible applications. As a result, agencies need to look for the relevant guidance for each application in this format. This is common to the traditional model of static content delivery, which pushes technical documents that cover many options and variations to an audience with varying needs.

One identified improvement was to deliver the NTD Sampling Manual not only in its current static format but also in a dynamic delivery format. Instead of pushing an entire manual of many possible applications to the audience, a dynamic delivery format allows a user to pull the specific content that is relevant to a specific application. This would be accomplished by developing an Internet-based interactive Web tool where the full content of the NTD Sampling Manual would be stored in terms of individual topics, but only the relevant topics would be dynamically assembled and presented to a user once the user specifies the profile of a specific application. The manual consists of a large number of pairs of questions/answers; therefore, the individual pairs of questions/answers would be a natural unit of presentation for the topics in the Web tool.

In addition to improving the delivery of the NTD Sampling Manual, FTA also wanted to make enhancements to the existing manual. Some of these were motivated by the desire to ensure that the sampling plans developed from the NTD Sampling Template are based on robust and precise sample data; some were motivated by the desire to give more options to transit agencies in developing sampling plans; and others were motivated by the desire to further reduce the reporting burden on transit agencies, particularly for small- and mid-size agencies. 
The objectives of this project include the following:

1. To investigate and implement the most feasible and beneficial enhancements to the NTD Sampling Manual and the NTD Sampling Template

2. To search for and implement the most appropriate approach to dynamic delivery of the NTD Sampling Manual.

The remainder of this report documents the project and is organized into four chapters and one appendix. Chapter 2 presents all potential enhancements identified by FTA and discusses separately those enhancements that were implemented and those that were not. Chapter 3 discusses alternative approaches to implementing dynamic delivery of the manual. Chapter 4 presents the implementation of the chosen approach to dynamic delivery and describes the resulting Web tool. Chapter 5 concludes the report. Finally, the appendix includes the new version of the full NTD Sampling Manual. 


\section{Chapter 2 - Enhancements to the Sampling Manual}

In addition to improving how the NTD Sampling Manual is delivered, FTA identified a number of enhancements to the manual that would serve NTD reporters even better. This section first lists all of the enhancements identified and then describes the enhancements that were implemented and those that were not. The revised new NTD Sampling Manual is included in the appendix.

\section{Enhancements Identified}

Identified enhancements to the manual are listed below. These enhancements are grouped into categories in terms of their primary motivation:

- To ensure that new NTD sampling plans are based on robust and precise sample data:

- Adding the requirement of minimum sample size of 50 for new sampling plans and for sample data used to develop new sampling plans

- Adding guidance on identifying and correcting errors in sample data for developing new sampling plans

- To provide more options to transit agencies in developing sampling plans:

- Adding the option of developing interval-based sampling plans

- Adding guidance for alternative sampling plans

- Adding the new modes recently recognized by the NTD

- To further reduce the reporting burden to transit agencies, particularly for small- and medium-size agencies:

- Condensing and simplifying guidance through dynamic delivery

- Adding small-system waivers. These waivers allow small systems to continue using ready-to-use sampling plans and give small systems the option of not reporting passenger miles traveled.

- Allowing combined sampling for different types of service for a given mode

- Allowing combined sampling for different bus modes for a given type of service

- To simplify the NTD Sampling Manual for all users. Specifically, the current version has a rather involved procedure for updating sampling plans over time.

The current project investigated each of these potential enhancements in terms of their feasibility and their pros and cons. Most of these enhancements were implemented, but several were not. In deciding which identified enhancement was to be implemented, the main criterion was how the potential benefits of an enhancement compared to its potential shortcomings. 


\section{Enhancements Implemented}

Most of the above-identified enhancements have been implemented into the new NTD Sampling Manual. Each is discussed below separately.

\section{New NTD Modes}

The NTD recently made several changes to the individual modes recognized for NTD reporting purposes; therefore, the new manual includes these new modes. A description of each new mode is listed below:

- A special form of demand response services is now recognized and called Demand Response-Taxi (DT). This mode is operated through taxicab providers and is always treated as purchased transportation in terms of service type.

- The traditional single motorbus mode is split into three separate bus modes: regular bus (MB), commuter bus (CB), and bus rapid transit (RB).

- The traditional light rail mode is split into streetcar rail (SR) and light rail (LR).

- The traditional monorail and automated guideway modes are combined into a single Monorail/Automated Guideway (MG) mode.

- Hybrid Rail (YR) is recognized as a new mode, which is for rail transit systems primarily operating entire routes on the National system of railroads, but not operating with the characteristics of commuter rail. This service typically operates light rail-type vehicles as diesel multiple-unit trains.

\section{Small System Waivers}

The NTD recently added the option of allowing small agencies with 30 or fewer vehicles operated in maximum service (VOMS) for all modes combined not to report passenger miles traveled to the NTD for any of their modes of service. This small-system waiver has been added to the manual.

In addition, the new manual also has added the option of allowing these small systems to continually use ready-to-use sampling plans as long as they remain small systems (i.e., VOMS for all modes combined $<=30$ ).

\section{Condensed and Simplified Guidance}

The NTD includes a large number of small- and mid-size transit systems, typically comprised of a few buses and a demand-response operation. These systems often find the whole sampling process, as well as NTD Sampling Manual, to be overwhelming. FTA felt that finding ways to condense and simplify the sampling guidance for these systems could be especially useful.

In terms of the manual itself, dynamic delivery, once implemented, will condense and simplify the manual for specific applications to all agencies. This is particularly effective for 
small agencies. Their operations are typically limited to regular bus service and demand response. As a result, they do not have to sift through non-applicable information.

In terms of the whole sampling process, however, it would be difficult to shorten or simplify it until FTA loosens some of its basic NTD statistical requirements for small agencies.

\section{Alternative Sampling Plans}

To meet FTA's statistical requirements to estimate unlinked passenger trips and passenger miles traveled, agencies have three options in terms of sampling plans:

- Ready-to-use sampling plans from the NTD Sampling Manual for those agencies that do not have previous NTD sample data.

- Template sampling plans that transit agencies can develop with their own NTD sample data using the NTD Sampling Template.

- Alternative sampling plans that transit agencies can ask qualified statisticians to develop and certify using the NTD sample data from these agencies.

Alternative sampling plans are unique in that they must be certified by a qualified statistician to meet FTA's statistical requirements. Ready-to-use sampling plans and template sampling plans are certified as long as transit agencies follow the guidance in the manual. To ensure the basic statistical quality of template sampling plans, they must meet the following five requirements:

1. A $25 \%$ margin of safety is built into any template sampling plans. If a given set of sample data results in an initial annual sample size of 100 , for example, the final sample size must be increased by at least $25 \%$ to a total of 125 .

2. The annual total sample size must be at least 50 for any new template sampling plan.

3. The sample data to be used to develop template sampling plans must have at least 50 samples.

4. Template sampling plans must be periodically checked for the need for revision based on information built into the NTD Sampling Manual.

5. Agencies must follow the guidance in the NTD Sampling Manual for sampling, collecting sample data, identifying and correcting errors in the sample data, and estimating annual totals from the corrected sample data.

The new manual has added a section of guidance to require alternative sampling plans to meet the first three requirements. The last two requirements are not explicitly included in the added section because they should be considered by the qualified statisticians during their certification process.

\section{Simplified Procedure for Revision of Template Sampling Plans}

The automatic certification of template sampling plans developed from the Sampling Template is conditional on the sampling plans being periodically checked for the need for 
revision due to a changed operating environment. The current manual includes a procedure for transit agencies to do that. However, the current procedure requires transit agencies to perform additional calculations beyond entering their sample data into the Sampling Template. At the same time, the guidance in the manual to help transit agencies follow this procedure is somewhat lengthy and complex. One reason for this rather involved and complex procedure is that it is based on determining whether the degree of statistical variation in the use of services has changed over time.

The new manual and its template have replaced this procedure with a simple one that does not involve calculations by transit agencies. The related guidance is brief and simple. The new procedure is based on an index calculated automatically by the Sampling Template once a set of sample data are entered.

\section{Guidance on Errors in Sample Data for Developing Sampling Plans}

The NTD Sampling Manual already includes guidance on identifying and correcting nonsampling errors in the sample data for estimating annual totals of unlinked passenger trips and passenger miles traveled but not for developing new sampling plans. Experience in working with transit agencies for developing new sampling plans using their own sample data shows that serious errors frequently occur in such sample data. In addition, many of these serious errors can be easily identified through simple checks. Once they are identified, transit agencies can go back to the original field data and make corrections. Experience also indicates that such serious errors typically lead to larger variances in the data. These inflated variances have several undesirable consequences:

- Transit agencies unnecessarily may be asked to revise their existing sampling plans.

- The suggested minimum sample size in a new sampling plan is significantly greater than what would be required.

- The stability in the annual minimum sample size is lost due to the minimum sample sizes for new sampling plans over time fluctuating significantly.

While these negative consequences do not result in FTA's statistical requirements not being satisfied, they unnecessarily increase the reporting burden of transit agencies.

\section{Option for Developing Interval-Based Sampling Plans}

The current NTD Sampling Manual provides guidance for transit agencies to develop sampling plans that require them to sample on random days during each week, month, or quarter and these days vary across weeks, months, or quarters. In contrast, the sampling plans in Circular 2710.1A for bus modes follow a structured interval pattern: every day, every other day, every third day, every fourth day, every sixth day, and every seventh day. In addition, these sample days are known to the transit agencies in advance for an entire report year. A third advantage is that transit agencies can choose samples with larger intervals (e.g., every sixth day) to reduce the number of sample days during a report year. Finally, many bus operators have been using these circular sampling plans for years. As a 
result, FTA wants to add the option of developing such structured sampling plans to the manual.

This project investigated the feasibility of adding this option, particularly to the Sampling Template. One issue was how to avoid creating confusion with two types of sampling plans that both are customized to agency conditions. The sampling plans developed from the current version of the Sampling Template are called "period-based sampling plans" and the newly-added structured sampling plans are called "interval-based sampling plans."

Another issue was how to determine the sample size requirement for each sampling interval from a set of sampling data entered into the Sampling Template. Facilitated by the work from a previous NCTR project on an investigation of the sampling plans in Circular 2710.1A, adding the option of developing interval-based sampling plans proved to be feasible. There are two limitations, however, to this option in terms of their applicability:

- Unlike period-based sampling plans, developing interval-based sampling plans requires at least two samples for every sample day in the sample data.

- Transit agencies can consider a wide range of efficiency options in developing periodbased sampling plans. One of these is to take advantage of the fact that the average passenger trip length (APTL) almost always varies significantly less than passenger miles traveled across vehicle trips. This efficiency option is called the "APTL option." Another efficiency option for period-based sampling plans is to sample separately for different parts of a service. One typical example for bus services is to group long routes separately from shorter routes. This is called the "grouping option." Transit agencies that want to use interval-based sampling plans would not be able to take advantage of the grouping option. The new NTD Sampling Template does not include the grouping option for interval-based sampling plans because interval-based sampling plans are day-based, and the grouping option would not help reduce sample size requirements.

\section{Minimum Sample Size of 50}

The current manual can produce period-based sampling plans whose annual sample size can be below 50 for some cases with $100 \%$ unlinked passenger trips being reported to the NTD. The annual sample size can be very small, even with the required $25 \%$ margin of safety. Commuter rail and commuter bus services are two examples of such cases. While these small sample sizes may be adequate to meet FTA's statistical requirements for estimating annual totals of passenger miles traveled for some situations, they may not produce estimates of the degree of variation in the resulting sample data that are precise enough with adequate confidence. The degree of variation in the sample data collected from a current sampling plan determines the sample size in future sampling plans. If this degree of variation does not have adequate precision and confidence levels, the resulting new sampling plans may not produce estimates of annual totals of passenger miles traveled that meet FTA's statistical requirements. FTA wanted a minimum annual sample size to be determined and implemented in the new manual. 
A literature search was conducted to determine the sample size for estimating variance to meet certain confidence and precision levels. This search identified equation (2.3) by Gupta and Gupta (1987) and was used to determine the required sample size for estimating variance to meet certain confidence and precision levels. The following shows the required sample size for two levels of precision and confidence for variance estimation:

\begin{tabular}{|c|r|r|}
\hline \multirow{2}{*}{ Precision of Variance } & \multicolumn{2}{|c|}{ Confidence of Variance } \\
\cline { 2 - 3 } & $\mathbf{9 5 \%}$ & $\mathbf{9 0 \%}$ \\
\hline $10 \%$ & 194 & 137 \\
\hline $20 \%$ & 50 & 35 \\
\hline
\end{tabular}

If the requirements imposed on annual totals of unlinked passenger trips and passenger miles traveled also apply to estimates of variance, the required minimum sample size would be 194. Experience indicates that the annual sample size for estimated annual totals to meet the $95 \%$ confidence and $10 \%$ precision levels can be much smaller than 194 for many transit agencies. If the precision level is reduced to $20 \%$ at the same $95 \%$ confidence level, the required minimum sample size would be just 50 .

FTA feels that this combination of $20 \%$ precision with $95 \%$ confidence is adequate. As a result, the new manual has implemented a minimum sample size of 50 for all period-based sampling plans, including ready-to-use sampling plans, template sampling plans, and alternative sampling plans. Because interval-based sampling plans are highly structured, with the longest sampling interval being limited to 7 days, the annual sample size will always be greater than 50 for all cases.

In addition to requiring all period-based sampling plans to have a minimum sample size of 50 , the new manual also imposes this minimum sample size on the sample data being used in developing sampling plans of any type, including both period-based and interval-based as well as template and alternative sampling plans. If a transit agency wants to develop new sampling plans but does not have adequate sample size in its own sample data, it will need to use a ready-to-use sampling plan for one year and use the sample data from the readyto-use sampling plan to develop new template sampling plans for future years.

The requirement for a minimum sample size of 50 ensures that more reliable and accurate estimates of variance are used in developing new sampling plans (alternative or template).

\section{Enhancements Not Implemented}

\section{Combined Sampling}

Recent changes to the NTD reporting rules split the single motorbus mode into three separate bus modes: regular bus (MB), commuter bus (CB), and bus rapid transit (RB). Under current NTD rules, separate sampling is required for each combination of mode and service type (directly operated or purchased). FTA was concerned that this split may increase the reporting burden on some agencies, especially if they have all three of these 
bus modes and they provide these services both directly and indirectly through purchased transportation contracts. Allowing combined sampling for all three modes of a given service type or combined sampling of different service types of a given bus mode may lessen the potential increase in reporting burdens.

Potential Effects. The extent of this potential increase in reporting burden may be not as big as might be expected. Needing six separate sampling plans for bus services may occur, but is likely to be rare. In addition, the statistical variation in APTL is likely much smaller for $C B$ and $R B$, particularly for $C B$. Consequently, the burden of data collection for these new modes is not likely to be close to the same magnitude as the base bus service in most cases. Separate sampling for each of these modes might mean additional work in creating sampling frames in the number of times sampling is done and in estimation. But if an agency has RB and $C B$, it may group its overall bus service and treat RB and $C B$ as separate groups. If this is the case, splitting the bus modes results in some extra data collection burden but no extra sampling burden.

If combined sampling across service type or across bus modes is an option, not a requirement, agencies may choose not to use the option if it is too much trouble, if they have good data from automatic passenger counters (APCs) for RB, etc. If that occurs frequently, combined sampling would benefit far fewer transit agencies than the full potential.

The burden of three separate bus modes for a given service type is acceptable for the vast majority of agencies. These transit agencies collect and report $100 \%$ counts of unlinked passenger trips (UPT) for all bus modes. The share of these agencies will continue to increase in the future. The main basis for the burden being acceptable for these agencies is that the combined total of annual sample sizes for all three bus modes of a given service type is likely not to exceed those in Circular $2710.1 \mathrm{~A}$ or is likely not to exceed them by much if greater than those in Circular 2710.1A.

However, the burden of three bus modes may not be acceptable for other transit agencies that sample, estimate, and report both unlinked passenger trips and passenger miles traveled. This assessment is based on the fact that the combined total of annual sample sizes for all three bus modes of a given service type is likely to exceed those in Circular 2710.1A considerably. The exact additional burden depends on each specific case and would increase with the number of bus modes for a given service type.

Based on these assessments, combined sampling across bus modes or across service types is not needed for transit agencies that report $100 \%$ UPT counts.

The question is what to do with agencies that do not report $100 \%$ UPT counts. Based on the increased burden from three bus modes, combined sampling should be allowed. On other hand, it should not be allowed because this contradicts FTA's desire of $100 \%$ counts of UPT being reported and implies that FTA does not take its statistical requirements of $10 \%$ precision and $95 \%$ confidence levels seriously. Also, the share of 
these agencies is expected to decrease over time and the requirements should be kept as simple as possible.

Shortcomings. Combined sampling has several shortcomings, one of which is the loss of data. Indicators involving data items required for directly operated services but not for purchased services would no longer be available. Similarly, data on unlinked passenger trips and passenger miles traveled would not be available separately for each of the new bus modes, defeating some of the benefit of splitting the traditional bus mode into three bus modes. In addition, managing the process and sampling and data collection can become significantly more difficult with combined sampling across service types because different service types are usually operated separately, potentially defeating the benefit of an overall smaller sample size from combining sampling. Combined sampling also may complicate contracts for purchased services in matters related to NTD.

\section{Waiver for the Minimum Sample Size of 50}

FTA sought to find ways to reduce the potential increase in reporting burdens as a result of the recent split of the traditional motorbus mode into three separate bus modes. One identified way was to waive the minimum sample size of 50 for some circumstances. However, investigation of the potential benefits and shortcomings of this proposed improvement resulted in not implementing it in the new manual for the following reasons:

- The number of qualifying agencies is likely to be small. Experience with sample data from a range of modes and agencies indicates that such a waiver is applicable mostly to commuter rail and commuter bus services that report $100 \%$ counts of unlinked passenger trips.

- The benefit of this waiver is extremely small in absolute terms for any qualifying agency. Suppose that the actual sample size is 30; the reduction from the waiver would be 20 , which would not be significant in terms of cost for data collection on an annual basis. 


\section{Chapter 3 - Alternative Content Management Systems}

The Web tool needs a content management system that stores the manual and assembles and delivers the appropriate content for specific usage profiles. This chapter discusses the search for the most appropriate content management system for the project. The first subsection summarizes key characteristics of the manual; the second and third subsections cover potential content management systems and WordPress as the chosen approach, respectively.

\section{Characteristics of the New NTD Sampling Manual}

Written in a question-and-answer (Q\&A) format, the new manual is a comprehensive guidance document for transit agencies to meet FTA's minimum statistical requirements of $10 \%$ precision and $95 \%$ confidence levels in the annual totals of unlinked passenger trips and passenger miles traveled they estimate through random sampling. It covers:

- All modes explicitly recognized by the NTD.

- Both types of service (directly-operated and purchased).

- All scales of operation (small, medium, and large).

- All patterns of operation (weekdays only, daily, etc.).

- All steps for getting estimates of annual totals (getting a sampling plan, collecting sample data, processing sample data, and estimating annual totals).

- All reporting practices ( $100 \%$ counts of unlinked passenger trips, etc.).

- A wide range of options for sampling efficiency.

- Availability of previous sample data for developing sampling plans.

- Two types of structure in sampling plans (period-based and interval-based).

- A range of sampling frequency (weekly, monthly, and quarterly) for period-based sampling plans.

- A range of sampling frequency (every day, every second day, every third day, every fourth day, every fifth day, and every sixth day) for interval-based sampling plans.

- A range of sampling plans in terms of who develops and certifies them (ready-to-use sampling plans, template sampling plans, and alternative sampling plans).

For a given agency, only a subset of the elements of the full manual may be relevant. For example, for agencies that operate only demand-response and regular bus services, elements related to other modes are irrelevant. For agencies that operate only fixed-route services, elements related to non-scheduled services are irrelevant. For each application of a given agency, a still narrower set of these elements may be relevant. For example:

- $100 \%$ counts of unlinked passenger trips are reported for demand-response but not for regular bus.

- Interval-based sampling plans may be more useful for regular bus, but period-based sampling plans may be more appropriate for demand response.

- Different staff may be responsible for different steps of getting estimates of annual totals, and each person would be interested only in the relevant step. 
The NTD Sampling Manual consists of 7 major sections, 21 subsections, 122 pairs of questions/answers, and 1 appendix. Each of these sections and pairs of questions/answers may be applicable to a range of agencies and their applications. While this is not a problem in searching for the most appropriate approach to dynamic delivery, it must be considered in designing the delivery tool. Also, each major section and subsection may cover many of the elements covered in the manual. As a result, it would not be meaningful to use these sections and subsections as the unit of dynamic delivery. Furthermore, even a single pair of question/answer may involve multiple elements for some of the pairs of questions/answers. For example, a question may cover certain guidance for non-schedule services but the answer differs slightly across different modes of non-scheduled services (i.e., demand response, vanpool, etc.).

\section{Potential Content Management Systems}

\section{Search Criteria}

The first step to searching for the most appropriate content management system was to identify the needs of the Web tool. Project researchers identified the following necessary capabilities for the Web tool:

(a) Able to assemble and present only the relevant topics of the manual to a user once he/she specifies the profile of a specific use through a user-interface within the Web tool.

(b) Able to be updated with relative ease, allowing the content of the manual to be edited within the Web tool, with changes propagating into a fully-revised manual in Word or PDF format. Alternatively, it should be easy to update the Web tool to reflect changes already made to the full manual.

(c) Able to provide users with:

(1) Access to the full version of the manual

(2) Access to and use of the Sampling Template

(3) Ability to browse the manual

(d) Able to allow users to save and print the dynamically-delivered content.

In addition to these desired capabilities, another search criterion was that the chosen content management system must be compatible with the following programming environment, indicated by FTA as compatible with the NTD website:

- PHP 4.3 (or greater; 5.0 or greater recommended)

- MySQL 4.1.2 (or greater; 5.0 or greater recommended)

- Apache with the mod_rewrite module enabled 


\section{Basic Logic behind Dynamic Delivery}

Once the general needs of the Web tool were understood, the next step was to understand the basic logic behind any dynamic delivery of the NTD Sampling Manual. This involved several aspects.

Profiling Questions. To get the dynamically-delivered content for a specific use of the manual from the Web tool, users must answer one or more profiling questions in a user interface. The project initially identified more than 20 potential profiling questions and grouped them into three categories:

- Pure Choice - Users are free to choose once they have evaluated their situation. The most obvious are service grouping and sampling frequency.

- Semi Choice - Users are largely free to choose, but their particular situation may prevent them from choosing certain options. These would include sampling units (e.g., one-way versus round vehicle trips), sampling structure (i.e., period-based versus interval-based), 100\% UPT by service group.

- Non Choice - Users do not have much freedom to choose among the options.

Only non-choice questions may be used as profiling questions. Among all potential profiling questions, nine of them were non-choice questions and are listed below:

1) Do you sample every year?

2) Is next year your mandatory sampling year? (Small agencies only are required to sample every third year.)

3) Have you made major changes to your service since you started using your current template sampling plan?

4) How many days of service do you provide every week?

5) Do you have $100 \%$ UPT by route?

6) What are you trying to do?

7) What is the mode of service?

8) Do you report $100 \%$ UPT?

9) You do not have own sample data?

The first five of these were not chosen for at least two reasons: 1) their different options would not affect much of the content delivered, and 2) they involve details of the process of obtaining estimates of annual totals and would not be clear to many users who are new to the NTD process. The last four of these were chosen as the profiling questions at this point and are shown in Table 1 along with their profiling options.

Unit of Topics. A natural selection of the unit of topics for dynamic delivery would be the individual pairs of questions/answers under ideal conditions. As described earlier, a single pair of question/answer may cover slightly different guidance for different options of a given profiling question, and this occurs to some pairs of questions/answers in relation to profiling by mode and by $100 \%$ UPT reporting. 
Table 1. Profiling Questions, Options, and Examples

\begin{tabular}{|c|c|c|c|c|c|}
\hline \multirow{2}{*}{ Profiling Questions } & \multirow{2}{*}{ Profiling Options } & \multicolumn{4}{|c|}{ Profiling Examples } \\
\hline & & 1 & 2 & 3 & 4 \\
\hline \multirow{4}{*}{$\begin{array}{l}\text { 1. What are you trying } \\
\text { to do? }\end{array}$} & General Directions & & & & \\
\hline & Sampling Plans & $\sqrt{ }$ & & & \\
\hline & Data Collection & & & $\sqrt{ }$ & $\sqrt{ }$ \\
\hline & Estimation & & & $\sqrt{ }$ & \\
\hline \multirow{7}{*}{$\begin{array}{l}\text { 2. What is the mode of } \\
\text { service? }\end{array}$} & Bus Modes & & & $\sqrt{ }$ & $\sqrt{ }$ \\
\hline & Commuter Rail & $\sqrt{ }$ & & & \\
\hline & Other Rail Modes & & & & \\
\hline & Other Scheduled Modes & & & & \\
\hline & Demand Response Modes & & & $\sqrt{ }$ & \\
\hline & Commuter Vanpool & & $\sqrt{ }$ & & \\
\hline & Other Non-Scheduled Modes & & & & \\
\hline \multirow{2}{*}{$\begin{array}{l}\text { 3. Do you report } 100 \% \\
\text { UPT? }\end{array}$} & Yes & $\sqrt{ }$ & $\sqrt{ }$ & $\sqrt{ }$ & \\
\hline & No & & & & \\
\hline \multirow{2}{*}{$\begin{array}{l}\text { 4. You do not have own } \\
\text { sample data? }\end{array}$} & Yes & & $\sqrt{ }$ & & \\
\hline & No & $\sqrt{ }$ & & & \\
\hline
\end{tabular}

To allow individual pairs of questions/answers to be the unit of topics, separating those questions whose answers cover more than one profiling option on mode and reporting of $100 \%$ UPT was considered. For example, for a question on non-scheduled services, one part of the answer covers guidance for demand-response (DR or DT), and the remainder covers guidance for vanpool. The answer for this original question would be separated into two pieces-one for demand-response services and one for vanpool. However, it was decided not to break these questions for two reasons: it would increase the total number of pairs of questions/answers significantly, and it would result in the new version of the manual appearing significantly different from the current version. Neither of these consequences is desirable.

As a result, the search for the best content management system treated the whole pair of question/answer as a delivery topic if the answer covers only one profiling option, but treated the different parts of the answer as different delivery topics if they cover different profiling options. For example, the guidance for a particular question in the manual may relate to agencies that report $100 \%$ counts of unlinked passenger trips in one part of its answer but to agencies that do not report 100\% counts in another part of its answer. In this case, only one of these two parts of the answer would be delivered, depending on whether an agency reports 100\% counts.

Profiling Logic. The profiling logic is what the Web tool will use in assembling the relevant topics of the manual in response to the particular use profile specified by the user. The profiling logic consists of the following aspects:

- A profiling question is not answered if none of its profiling options is chosen. For example, the user in profile example 2 in Table 1 did not answer profiling question 1.

- Nothing would be delivered if the user does not answer any profiling question. 
- Only a portion of the manual would be delivered if the user answers just one question and the delivered content may be reduced further as the user answers more profiling questions.

- When a profiling question is answered, the amount of delivered content increases with more profiling options chosen for the given profiling question.

- Choosing all profiling options for a given profiling question is equivalent to the profiling question not being answered.

The user in profiling example 1 in Table 1 probably operates commuter rail service for the first time, knows exactly how many riders use its service, does not have previouslycollected sample data because the service is new, and needs to get a sampling plan to estimate the annual total passenger miles traveled (PMT) for this new service. The user in profiling example 2, on the other hand, operates vanpool service, also knows exactly how many customers use its service every day, has sample data collected from previous years, and wants to learn all aspects of the process for getting annual total PMT for the service.

\section{Open-Source Solutions}

Once the needs of the Web tool and the profiling logic for accomplishing dynamic delivery were understood, the next step of the search was to consider off-the-shelf software, either commercial or non-commercial. Typically, content management software uses $\mathrm{XML} /$ database schemas to deliver dynamically-generated material. Using XML tags, the precise and relevant sections of the document can be parsed and "delivered" to a user. Based on research by CUTR's GIS group, however, these off-the-shelf products do not have the capability to dynamically deliver the NTD Sampling Manual under the profiling logic described above.

Without successfully identifying any off-the-shelf product for further consideration, the next step was to consider open-source products. Open-source applications are fully-developed products that can be altered and customized to meet unique needs of specific Web applications. Two potential open-source solutions were identified for further consideration.

WordPress. WordPress is a database-driven content management system (CMS) for websites. While WordPress is widely used for managing and delivering Internet content for specific websites, using it to dynamically deliver the sampling manual turned out not to be as easy as initially thought.

To develop the Web tool, the manual would need to be entered into the WordPress database (MySQL) in terms of individual pairs of questions/answers. The profiling logic described earlier would be followed to tag each pair of question/answer using a tagging system employed by the WordPress CMS. Once the eventual Web tool is complete, it can parse and dynamically deliver the individual topics in response to user profiles entered through a user interface. 
One problem was that the default tagging system offered by WordPress is a simple taxonomy that can allow entire WordPress sections to be tagged. While the unit of topics for dynamic delivery can be parts of an answer for some questions in the manual, as discussed earlier, individual questions and their entire answers are the smallest sections that WordPress recognizes. As a result, the eventual Web tool based on WordPress can deliver only questions and their whole answers but not parts of their answers.

Currently, tagging parts of an answer is feasible when a related third-party plug-in is used. However, the plug-in may not be updated to support future versions of WordPress. Consequently, as WordPress evolves and changes, this third-party plug-in built into the current Web tool may stop working. This is a real possibility, as WordPress is regularly updated, and without the third-party developer continuing to support the application, the plug-in used for this project may become out of date and no longer work. If this happens, more programming might be necessary for the Web tool to work. As a result, using this plug-in was not chosen as a solution to the tagging problem.

Beyond this tagging problem for dynamically delivering sub-answers, it is relatively easy to allow the full manual to be delivered in entirety or to be browsed for individual sections, subsections, or even individual pairs of questions/answers. Updating the Web tool in response to possible future changes to the manual requires that these changes first be made to the manual in Word and modifications then be made to the database and its tags accordingly. Printing only the dynamically-delivered content is also feasible.

LimeSurvey. LimeSurvey is an open-source product for designing dynamic survey instruments on the Internet. To use it for the eventual Web tool, LimeSurvey would use question tags that reveal and hide portions of the manual based on the profiling questions and options specified by its user. LimeSurvey is similar to WordPress in that it stores and organizes its data in a MySQL database. The advantage of LimeSurvey over WordPress is that it allows sub-answers of individual questions be tagged. As a result, the topic unit for dynamic delivery would be sub-answers rather than individual questions and their entire answers. Relative to WordPress, however, LimeSurvey has a number of significant shortcomings:

- It lacks flexibility in the appearance of the user interface for profiling questions and options, as well as the appearance of the content delivered.

- It is not well-suited for browsing the manual for sections, subsections, and individual pairs of questions/answers.

- It is extremely complex to update the Web tool in response to future changes made to the manual; editing the manual content in the database and related tags requires technical skills and familiarity with LimeSurvey.

- Printing dynamically-delivered contents needs to be programmatically developed. 


\section{WordPress as the Chosen Approach}

A Web tool based on LimeSurvey would be able to dynamically deliver the content of the manual as small as sub-answers of individual questions; however, using LimeSurvey to develop the Web tool requires customized programming and high levels of technical skills, not only for the dynamic delivery capability of the Web tool, but also for its other desired capabilities.

On the other hand, a Web tool based on WordPress would not be able to dynamically deliver the content of the manual as small as sub-answers. However, it is able to dynamically deliver individual questions and their entire answers as well as to provide the other desired capabilities with relative ease. These capabilities were described in the previous section as the search criteria for the best content management system.

As a result, WordPress was chosen as the content management system for developing the Web tool. This choice meant that the unit of topics for dynamic delivery had to be changed from sub-answers to entire answers for all questions in the manual. When compared to the technical complexity of using LimeSurvey for both developing and updating the eventual Web tool, this loss of detail in the unit of delivery topics was considered to be relatively small. 


\section{Chapter 4 - WordPress-Powered Dynamic Delivery}

With the general content management system chosen, the next step was to develop the Web tool. This chapter presents its development, the protocol for transferring and updating the tool, and its usage. The section on usage serves as a guide to using the Web tool.

\section{Developing the Web Tool}

\section{Design}

The design of the Web tool followed the principles of inclusiveness and simplicity. Inclusiveness refers to the need for the Web tool to have a range of options for the manual to be delivered to users, including the capabilities of dynamic delivery, semi-dynamic browsing and searching, and static downloading. This range of delivery options is meant to meet different needs of users. The capability of semi-dynamic browsing includes a range of options, including browsing by whole sections, by subsections, and by individual pairs of questions/answers. Semi-dynamic searching allows the user to search the entire manual by entering desired keywords. Finally, the download capability allows the following to be downloaded:

- Full manual

- Sampling Template

- A table of random numbers (Appendix 98 in the manual)

- Each appendix of sample tables for data collection

Simplicity refers to the appearance and features of the website being as simple as possible under the condition that the desired capabilities are available.

While the final design calls for the Sampling Template being made available as a downloadable Excel file, two alternatives were considered early on: embedding the Excel file into the Web tool, and programming the functionality of the template into the Web tool. Neither alternative was chosen for various reasons:

- Embedded. Embedding an interactive Excel workbook with multiple linked worksheets has been technically feasible using a Microsoft product called Office Web Components. The advantages of an embedded Sampling Template are clear: it avoids the need to download the file, and it is accessible from anywhere with Internet access. However, Microsoft will not to release new versions of this product by 2012 and has ended its standard support of the previously-released versions. Embedding such a complex Excel workbook is still feasible, but it requires that the workbook be hosted at Microsoft's SkyDrive site.

- $\quad$ Programmed. Programming the functionalities of the entire Sampling Template into the Web tool is feasible and has advantages over both downloading and embedding. However, it would require resources beyond the scope of the current project. 


\section{Build}

Before the design was implemented to build the Web tool using WordPress, the following tasks were completed first:

1. Select the programming environment.

2. Break the manual into individual topics for dynamic delivery.

3. Identify profiling parameters and the logic involved.

4. Link these profiling parameters to the individual topics in the manual based on the logic.

The first three tasks were done before WordPress was chosen as the content management system for building the Web tool. The individual topics are individual pairs of questions/answers. The profiling parameters are four profiling questions and a set of options for each profiling question. The logic requires the Web tool to deliver the common content when more than one profiling question is answered and to deliver the combined content when more than one profiling option is chosen for each given profiling question.

The fourth task involved linking the profiling questions and options to all individual pairs of questions/answers in the form of a matrix. Table 2 shows one part of this matrix for the questions on the ready-to-use sampling plans included in Subsection 43 of the manual. For a given manual question and a given profiling question, the primary tags differ from the filling tags as follows:

- $\quad$ Primary Tags. The guidance in the answer to the manual question would be selected for dynamic delivery only when at least at least one profiling option with a primary tag is selected. Consider manual question $\$ 43.01$ and the profiling question of "What are you trying to do?" Its guidance would be selected if the user selects the profiling option of "Sampling Plans" but would not be selected if the user selects any other profiling option of this profiling question.

- $\quad$ Filling Tags. The guidance in the answer to the manual question would be selected for possible delivery regardless of which profiling option of this profiling question is selected. Consider manual question $\$ 43.01$ and the profiling question of "Do you report 100\% UPT?" Its guidance would be selected if the user chooses to answer this manual question, independent of the profiling option selected.

Primary tags are separately defined from filling tags only for building the Web tool. The users of the Web tool will not see the existence of any tags. 
For the Web tool to be able to dynamically deliver the manual, the individual pairs of questions/answers in the manual must be manually entered into the database of WordPress. In addition, each pair of these questions/answers in the database must be manually tagged as described above. Once the database of questions/answers was created and tagged properly in WordPress, the last step was to build the user interface for the user to answer the profiling questions and select profiling options.

\section{Review}

The review of the draft Web tool focused on how the delivered manual content appeared and on the simplicity and functionality of the tool. The manual content delivered through the draft Web tool differed quite significantly from that in the actual manual. One spacing issue was that too much vertical space existed in the delivered text and tables. This spacing issue resulted when the original manual content in Word format was copied and pasted into the WordPress database. On one hand, it is desirable to have the delivered content looking similar to what is in the actual manual. On the other hand, it is undesirable to make too much adjustment to the WordPress after the actual manual content is pasted into it because such adjustment complicates future updates to the tool if the manual is revised in the future. In the end, adjustments were made to reduce the vertical spaces.

The design of the draft Web tool was reviewed to improve simplicity and functionality. Several issues were identified and addressed. The most notable was the addition of the option to using the manual through searching by keywords.

Test

The draft Web tool was tested with two levels of testing. One level was to determine if the Web tool delivers what was designed in the tag matrix between individual manual questions/answers and the profiling questions and options. This matrix was developed before building the Web tool. The objective of this level of testing was to see if any errors were made relative to the matrix when each of the manual questions/answers was manually tagged within WordPress. Once this level of testing was complete, the tags in the WordPress database of the questions/answers were corrected when any errors were identified.

The other level of testing was to determine if any errors were made in developing the tag matrix between individual manual questions/answers and the profiling questions and options. Before conducting the second level of testing, the following two steps of preparation were taken:

1. A variety of possible scenarios of the manual guidance being used was prepared. Each scenario was represented by the specific profiling questions to be answered and the specific profiling options selected for each answered profiling question.

2. For each of these scenarios of usage, the corresponding guidance relevant for this scenario was established. 
After these were prepared, the second level of testing was done in two steps:

1. Each of these scenarios of usage was entered into the Web tool through the user interface in terms of the profiling questions and options.

2. The dynamically-delivered manual content for each of the entered scenario of usage was then compared to the guidance that was thought to be relevant for this scenario.

Once these two steps were complete, the Web tool was revised again. Specifically, the tagging matrix was adjusted when any inappropriate tags were identified. The tags in the WordPress database of the manual questions/answers were revised again according to the adjusted tagging matrix.

\section{Transferring and Updating the Web Tool}

\section{Transfer}

The Web tool has been hosted at a temporary site at http://ntd.transitgis.org/ since its development. After this project ends, this temporary site will continue to be used until FTA moves it to its NTD site, at which time FTA's contractor for operating, maintaining, and developing its NTD site will transfer and take over the Web tool. As mentioned before, the Web tool has been developed within a programming environment that is compatible with the NTD website. While some level of programmatic and software skills are required to transfer the Web tool from its current site to the NTD site, it should within the FTA contractor's skills and capacity.

\section{Updating}

In the case where the new version of the manual is revised in the future, the Web tool would need to be updated accordingly if it is still active in the NTD site. FTA's contractor for NTD operation, maintenance, and development will need to carry out the update.

WordPress enables users to seamlessly update its database by creating or editing questions and assigning tags. The following explains how to use these features and how to make additional changes to the NTD manual as contained in the Web tool as needed.

Creating New Questions. Follow these steps to add new questions/answers to the WordPress database:

1. Login to the NTD WordPress website: <base URL>/wp-admin/.

2. Once logged in, hover over "Posts" on the navigation bar, and click "Add New."

3. Along with the title (i.e., the question) and body (i.e., the answer), the new post can be assigned to a tag (described under Assigning Tags later). The screenshot in Figure 1 illustrates the "Add New Post" page.

4. The question will be entered into the "Enter title here" text box, and the corresponding answer can be written or copied and pasted into the large text box below the editor menu. 
5. To copy a question from the Word version of the manual, copy the question title (including the section number), and paste into the "Enter title here" text box. Next, copy the answer portion from the Word document and paste it into the body text box in WordPress.

6. A completed post can then be previewed and published via the "Publish" widget located in the top-right portion of the page. "Preview" allows one to view a draft in real-time, while "Publish" makes the post live.

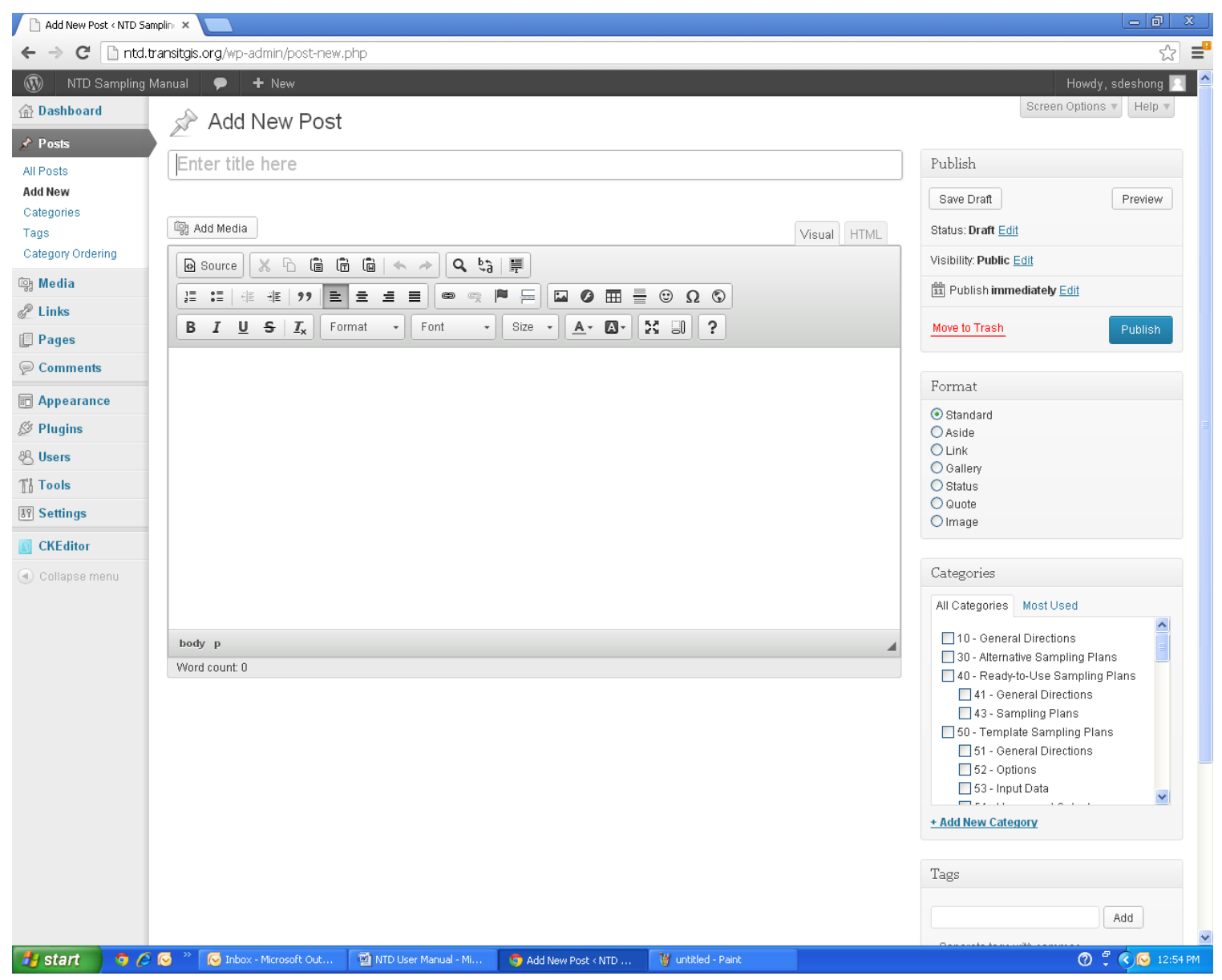

Figure 1. Creating New Questions

Editing a Question. Follow these steps to edit an existing question and its answer:

1. From the "Posts" menu, all the questions/answers previously published in WordPress can be accessed. To edit a post, hover over a title and click "Edit" ("Quick Edit" will be discussed later). The illustration in Figure 2 highlights this feature.

2. The edit feature allows for minor changes such as changing font, adding an image, or one can copy an excerpt from the NTD Manual and paste it into the WordPress editor.

3. Once finished editing a post, changes can be previewed and the post can be updated via the "Publish" widget as described under Creating New Questions. 


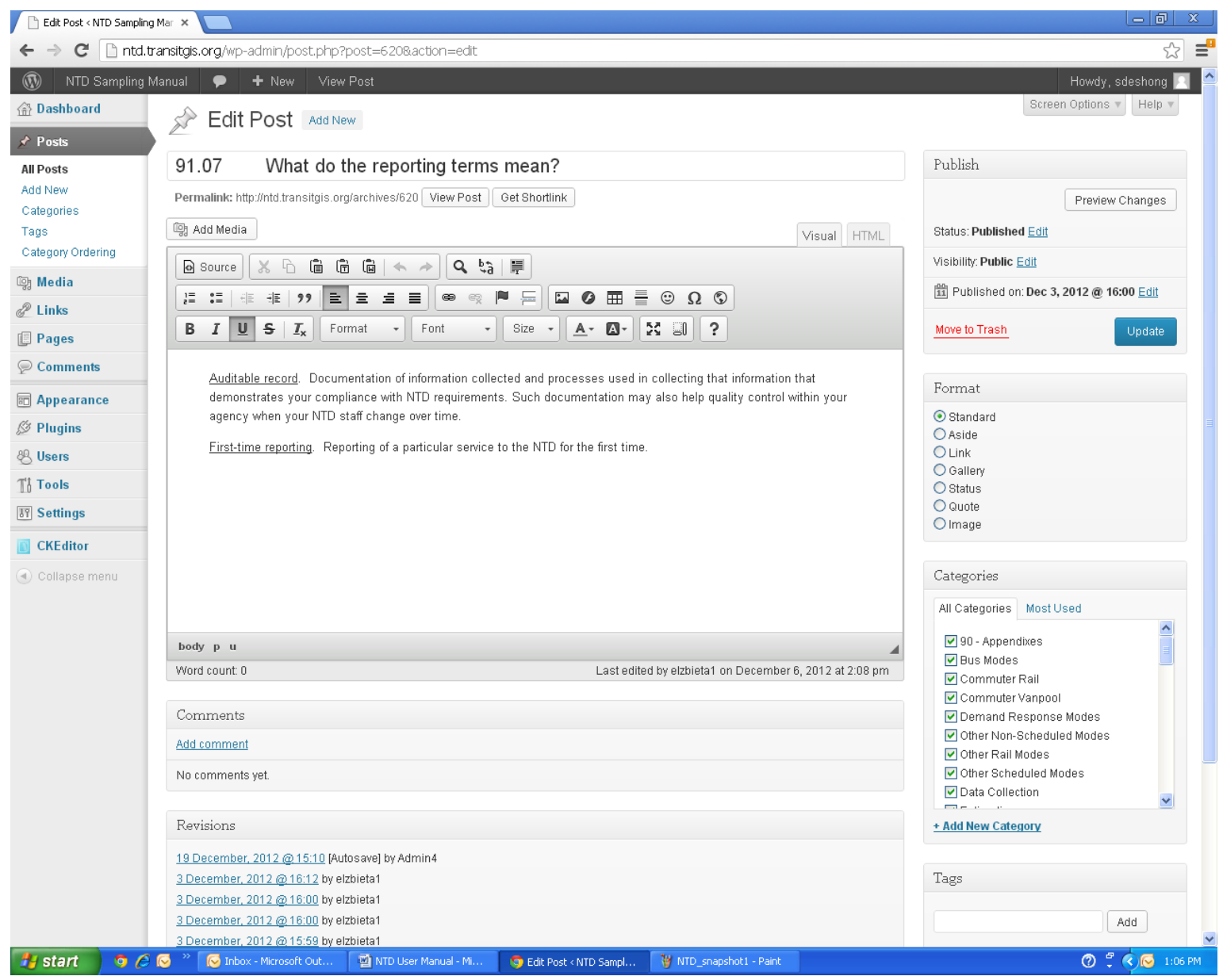

Figure 2. Editing a Question

Assigning Tags. Follow these steps to tag new or modified questions/answers:

1. In WordPress parlance, "tagging a post" means assigning it to a particular tag in the tag matrix discussed earlier. This can be done quickly and easily through the "Quick Edit" function (Figure 3).

2. From the "Posts" menu, highlight the entry that will be tagged and click "Quick Edit" below the title.

3. Click checkboxes in the "Categories" section for each section number in the NTD Manual the post corresponds. Ignore the "Tags" section.

4. Click "Update" once finished. 


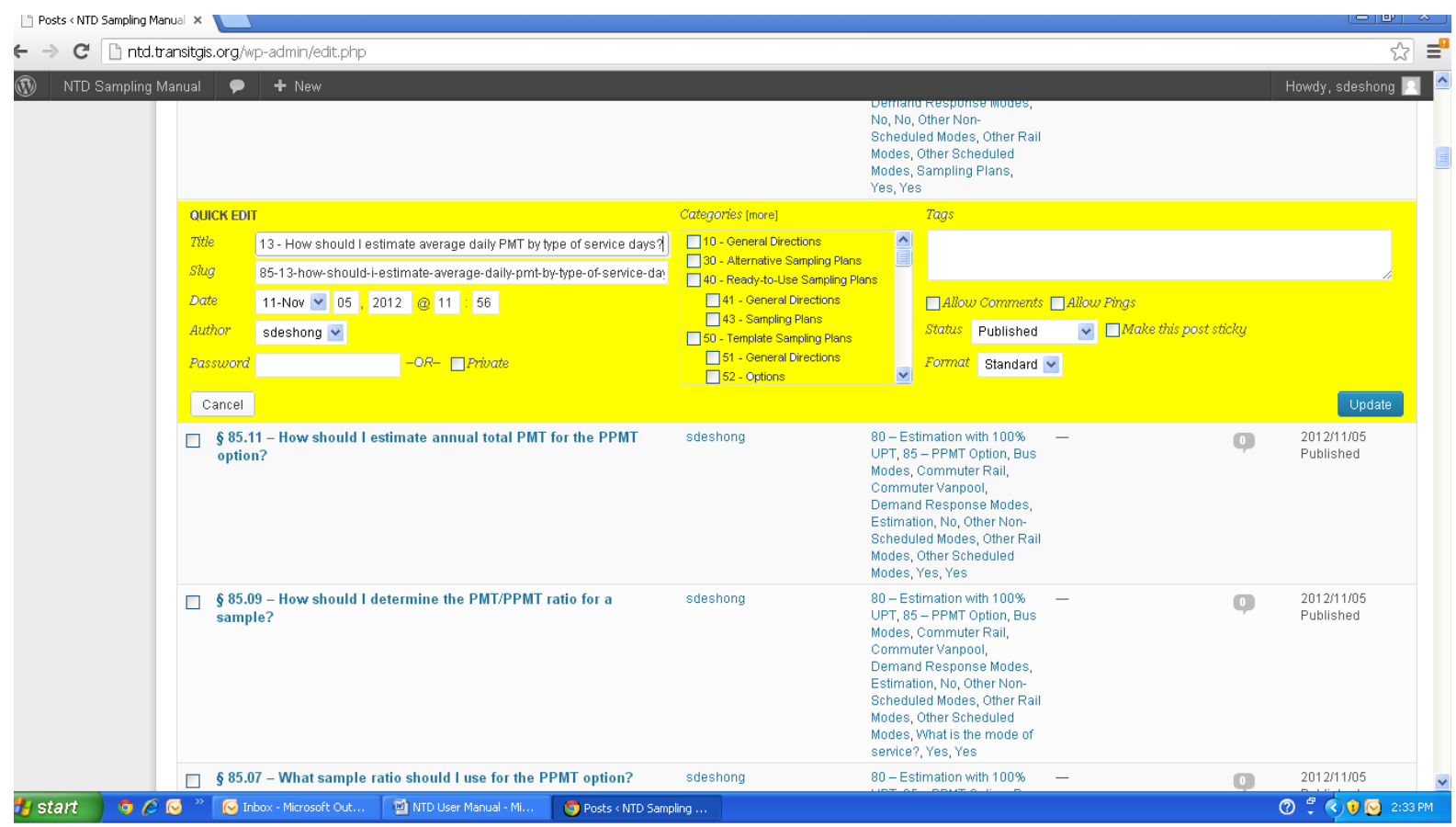

Figure 3. Assigning Tags

Deleting a Question. From the "Posts" menu, hover over a question that needs to be deleted, and click the "Trash" link below the title.

\section{Using the Web Tool}

This section is written in the form of a user guide. It also is available as a separate PDF file through the Web tool under About the Manual button.

If you need help with viewing the NTD Sampling Manual through this Web tool, this is the right place for you. This guide is organized into three sections:

1. Summary of the content of the manual and its main characteristics.

2. Description of the Web tool in terms of its home page and each of its options for viewing the manual.

3. Examples of using the Web tool to get exactly what you need for your specific profiles of using the manual.

\section{Summary of the Manual}

Written in a question-and-answer (Q\&A) format, the NTD Sampling Manual is a comprehensive guidance document to assist you to meet FTA's minimum statistical requirements. The requirements entail $10 \%$ precision and $95 \%$ confidence levels in the annual totals of unlinked passenger trips and passenger miles traveled, which you estimate for the NTD through random sampling. The full manual consists of 7 major sections, 21 subsections, 122 pairs of questions/answers, and 1 appendix. It covers: 
- All modes explicitly recognized by the NTD.

- Both types of service (directly-operated and purchased).

- All scales of operation (small, medium, and large).

- All patterns of operation (weekdays only, daily, etc.).

- All steps for getting estimates of annual totals (getting a sampling plan, collecting sample data, processing sample data, estimating annual totals).

- All reporting practices ( $100 \%$ counts of unlinked passenger trips, etc.).

- A wide range of options for sampling efficiency.

- Availability of own sample data for developing sampling plans.

- Two types of structures in sampling plans (period-based and interval-based).

- A range of sampling frequency (weekly, monthly, and quarterly) for period-based sampling plans.

- A range of sampling frequency (every day, every second day, every third day, every fourth day, every fifth day, and every sixth day) for interval-based sampling plans.

- A range of sampling plans in terms of who develops and certifies them (ready-to-use sampling plans, template sampling plans, and alternative sampling plans).

For each of you, only a subset of these elements of the full manual may be relevant. If you operate only demand-response and regular bus services, for example, those elements about other modes are irrelevant. If you operate only fixed-route services, those elements related to non-scheduled services are irrelevant. For your specific applications, a still narrower set of these elements may be relevant. For example:

- You report $100 \%$ UPT for demand-response but not for regular bus.

- Interval-based sampling plans may be more useful for your regular bus service, but period-based sampling plans may be more appropriate for your demand-response service.

- Different persons on your NTD staff may be responsible for different steps of getting estimates of annual totals, and each person would be interested only in the relevant step.

This Web tool is designed to help you select only the relevant portion of the full manual for your agency and for the specific need of each of your application.

\section{Home Page}

The home page of the Web tool is simple by design. It lists the main options of viewing the content from the manual. Figure 4 shows how the home page appears on the Internet. 


\section{NTD Sampling Manual}

Dynamic Delivery Prototype

\section{Home Filter the Manual Browse the Manual Download the Manual Search the Manual About the Manual}

U.S. Department of Transportation

Federal Transit Administration

National Transit Database Sampling Manual

Proudly powered by WordPress

Figure 4. Home Page of the Web Tool

Filtering the Manual

The "Filter the Manual" function of the Web tool gives you the most flexible and dynamic way for viewing the manual. After clicking the "Filter the Manual" button, you get a Web page that is split into left-right columns, as shown in Figure 5. The narrower left side hosts the user interface for you to enter the specific profile. The user interface consists of four profiling questions and the profiling options for each profiling question. The wider right side shows the full content of the manual in terms of all individual questions/answers. To narrow the content delivered on the right side, you must answer at least one profiling question by selecting at least one of its profiling options. 


\section{NTD Sampling Manual}

Dynamic Delivery Prototype

\section{Home Filter the Manual Browse the Manual Download the Manual Search the Manual About the Manual}

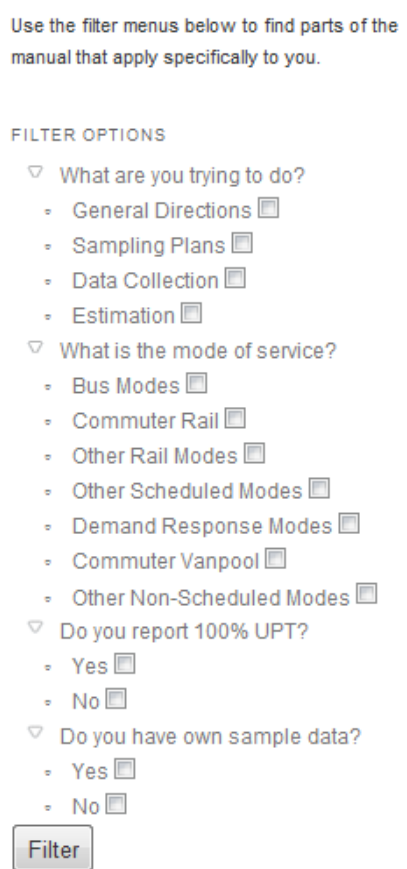

\section{$\S 10.01$ - What is the purpose of this Sampling} Manual?

(a) It suggests procedures for obtaining annual data on unlinked passenger trips (UPT) and passenger miles traveled (PMT) for the National Transit Database (NTD) through random sampling according to the requirements in the NTD Reporting Manual.

(b) In the event of a conflict between the requirements in the Reporting Manual and this Sampling Manual, the requirements of the Reporting Manual are definitive. (c) When it is possible to obtain annual UPT and PMT data according to the requirements in the Reporting Manual by using other methods not specified in this Sampling Manual, then you may do so.

\section{$\S 10.03$ - What procedures does this Sampling}

\section{Manual cover?}

(a) Development of sampling plans with two options:

(1) Section 40 on ready-to-use sampling plans

(2) Section 50 on agency-developed template sampling plans with agency sample data.

(b) Collection of sample data in Section 60 .

(c) Estimation of annual service-consumed data with two options:

(1) Both UPT and PMT if you do not report $100 \%$ counts of UPT

(2) PMT if you report $100 \%$ counts of UPT.

(d) Section 30 specifies several requirements for alternative sampling plans that template sampling plans must also meet.

(e) Figure 10.01 shows how these procedures and options relate to each other.

\section{Figure 5. Filtering the Manual}

Table 3 lists the profiling questions and their respective profiling options in the user interface. The last column briefly explains each profiling option. The bottom of the table describes the three types of sampling plans mentioned in explaining the profiling options.

\section{Examples of Dynamically Delivering the Manual}

Before you examine any examples shown in Table 4, it is essential for you to fully understand the logic behind the user interface and how the interface is linked to the content of the manual. This logic helps you correctly specify your usage profiles in the user interface to get what you need.

Logic behind the User Interface. The profiling logic is what the Web tool uses in assembling the relevant topics of the manual in response to the particular use profile that you specify. The profiling logic consists of the following aspects:

- A profiling question is not answered if you do not choose any profiling option. For example, the user in profile example 5 in Table 4 did not answer question 1. 
- Nothing would be delivered if you do not answer any profiling question.

- Only a portion of the manual would be delivered if you answer just one question and the delivered content is reduced further as you answer more questions.

- When a profiling question is answered, the amount of delivered content increases with more profiling options chosen for the given profiling question.

- Choosing all profiling options for a given profiling question is equivalent to the profiling question not being answered.

Table 3. Profiling Questions and Profiling Options for the User Interface

\begin{tabular}{|c|c|c|}
\hline Profiling Questions & Profiling Options & Notes \\
\hline \multirow{4}{*}{$\begin{array}{l}\text { 1. What are you } \\
\text { trying to do? }\end{array}$} & General Directions & Overall directions to the full manual \\
\hline & Sampling Plans & \multirow{3}{*}{$\begin{array}{l}\text { These are the major steps of getting } \\
\text { estimates of annual totals of UPT and PMT. }\end{array}$} \\
\hline & Data Collection & \\
\hline & Estimation & \\
\hline \multirow{7}{*}{$\begin{array}{l}\text { 2. What is the mode } \\
\text { of service? }\end{array}$} & Bus Modes & MB, CB, RB, etc. \\
\hline & Commuter Rail & CR \\
\hline & Other Rail Modes & Heavy rail, light rail, streetcar rail, etc. \\
\hline & Other Scheduled Modes & Ferryboat \\
\hline & Demand Response Modes & Regular (DR) or by taxi (DT) \\
\hline & Commuter Vanpool & Vanpool service for commuters \\
\hline & Other Non-Scheduled Modes & Jitney services, etc. \\
\hline \multirow{2}{*}{$\begin{array}{l}\text { 3. Do you report } \\
100 \% \text { UPT? }\end{array}$} & Yes & \multirow{2}{*}{$\begin{array}{l}\text { You can sample far less if you report } 100 \% \\
\text { UPT and only estimate PMT }\end{array}$} \\
\hline & No & \\
\hline \multirow{2}{*}{$\begin{array}{l}\text { 4. Do you have own } \\
\text { sample data? }\end{array}$} & Yes & Use template or alternative sampling plans \\
\hline & No & Should use ready-to-use sampling plans \\
\hline \\
\hline \multicolumn{3}{|c|}{$\begin{array}{l}\text { Notes: } \\
\text { 1. UPT = unlinked passenger trips. }\end{array}$} \\
\hline \\
\hline \multicolumn{3}{|r|}{ t year } \\
\hline \multicolumn{3}{|r|}{ ticians develop and certify with your ow } \\
\hline \multicolumn{3}{|r|}{$\begin{array}{l}\text { al provides for you to use temporally if you do not } \\
\text { ar for various reasons. }\end{array}$} \\
\hline
\end{tabular}

Examples. Table 4 summarizes examples of how you may filter the manual through the Web tool for five potential cases of using the manual:

1. You will start operating commuter rail service next year and will know exactly how many riders use the service, but you do not have previously-collected sample data because the service is new. It is a couple of months before next year starts, and you need to get a sampling plan now for estimating the annual total PMT for next year so you can plan ahead for the staffing needs of data collection.

2. You operate regular bus services only, you collect and report $100 \%$ UPT, and you have been sampling for several years; hence, you have your own sample data. 
What you want is all of the content in the manual that is relevant for your operation and NTD reporting practices. As a result, you choose all steps of the overall process of obtaining estimates of annual total PMT for your bus service.

Table 4. Illustrative Examples of Filtering the Manual

\begin{tabular}{|c|c|c|c|c|c|c|}
\hline \multirow{2}{*}{ Profiling Questions } & \multirow{2}{*}{ Profiling Options } & \multicolumn{5}{|c|}{ Examples } \\
\hline & & 1 & 2 & 3 & 4 & 5 \\
\hline \multirow{4}{*}{$\begin{array}{l}\text { 1. What are you trying } \\
\text { to do? }\end{array}$} & General Directions & & $\sqrt{ }$ & & & \\
\hline & Sampling Plans & $\sqrt{ }$ & $\sqrt{ }$ & & & \\
\hline & Data Collection & & $\sqrt{ }$ & & & \\
\hline & Estimation & & $\sqrt{ }$ & $\sqrt{ }$ & $\sqrt{ }$ & \\
\hline \multirow{7}{*}{$\begin{array}{l}\text { 2. What is the mode of } \\
\text { service? }\end{array}$} & Bus Modes & & $\sqrt{ }$ & $\sqrt{ }$ & $\sqrt{ }$ & $\sqrt{ }$ \\
\hline & Commuter Rail & $\sqrt{ }$ & & & & \\
\hline & Other Rail Modes & & & & $\sqrt{ }$ & \\
\hline & Other Scheduled Modes & & & & & \\
\hline & Demand Response Modes & & & $\sqrt{ }$ & $\sqrt{ }$ & $\sqrt{ }$ \\
\hline & Commuter Vanpool & & & & $\sqrt{ }$ & \\
\hline & Other Non-Scheduled Modes & & & & & \\
\hline \multirow{2}{*}{$\begin{array}{l}\text { 3. Do you report } 100 \% \\
\text { UPT? }\end{array}$} & Yes & $\sqrt{ }$ & $\sqrt{ }$ & $\sqrt{ }$ & $\sqrt{ }$ & $\sqrt{ }$ \\
\hline & No & & & & $\sqrt{ }$ & \\
\hline \multirow{2}{*}{$\begin{array}{l}\text { 4. Do you have own } \\
\text { sample data? }\end{array}$} & Yes & & $\sqrt{ }$ & & & \\
\hline & No & $\sqrt{ }$ & & & & $\sqrt{ }$ \\
\hline
\end{tabular}

3. Your agency used template sampling plans for both of your regular bus and demand-response services during the report year that just passed. Your agency collected $100 \%$ UPT and will report it to the NTD for both modes. The staff person in charge of obtaining annual total PMT for both modes has since left. It is time to estimate the annual totals of PMT for the two modes using the sample data collected by the previous staff person from last report year. You are the new staff person in charge of preparing the estimates, and you want to learn exactly what you need to do.

4. You are part of a large transit agency that operates regular bus, commuter bus, light rail, regular demand-response, and vanpool services. You and several other colleagues are involved in the NTD process at your agency. Your agency collects and reports $100 \%$ UPT all of these modes except light rail. Your NTD-related job is to estimate annual totals of UPT and PMT for all of these modes using sample data collected by your NTD colleagues. You want to have all of the estimationrelated content in the manual for all of the modes your agency operates. You do not choose an answer to the last profiling question because it is irrelevant to the estimation-related content in the manual.

5. You provide both regular bus and demand-response services, but have only 25 vehicles operated in maximum service. As a result, you qualify for the smallagency waiver-that is, you do not have to report PMT data to the NTD. In fact, you have not been sampling for several years and, hence, you do not have your 
own usable sample data. But you have been asked by other transportation partners in the region to start reporting PMT data so that the region can qualify for more Section 5307 funds. As a result, you want to learn more about what would take to report PMT data before making the final decision. The good thing is that you have been collecting and reporting 100\% UPT and will continue to do so in the future. You choose not to answer the first profiling question because you want guidance in the manual covering all of these steps.

\section{Browsing the Manual}

The Web tool gives you the option to browse manual for its sections, subsections, or individual pairs of questions/answers.

Sections. When you move your mouse curser over the "Browsing the Manual" button, the section numbers and titles appear as a drop-down menu, as shown in Figure 6. To view the entire content for a given section, just click the title of the section in the dropdown menu. All individual questions/answers for this section appear.

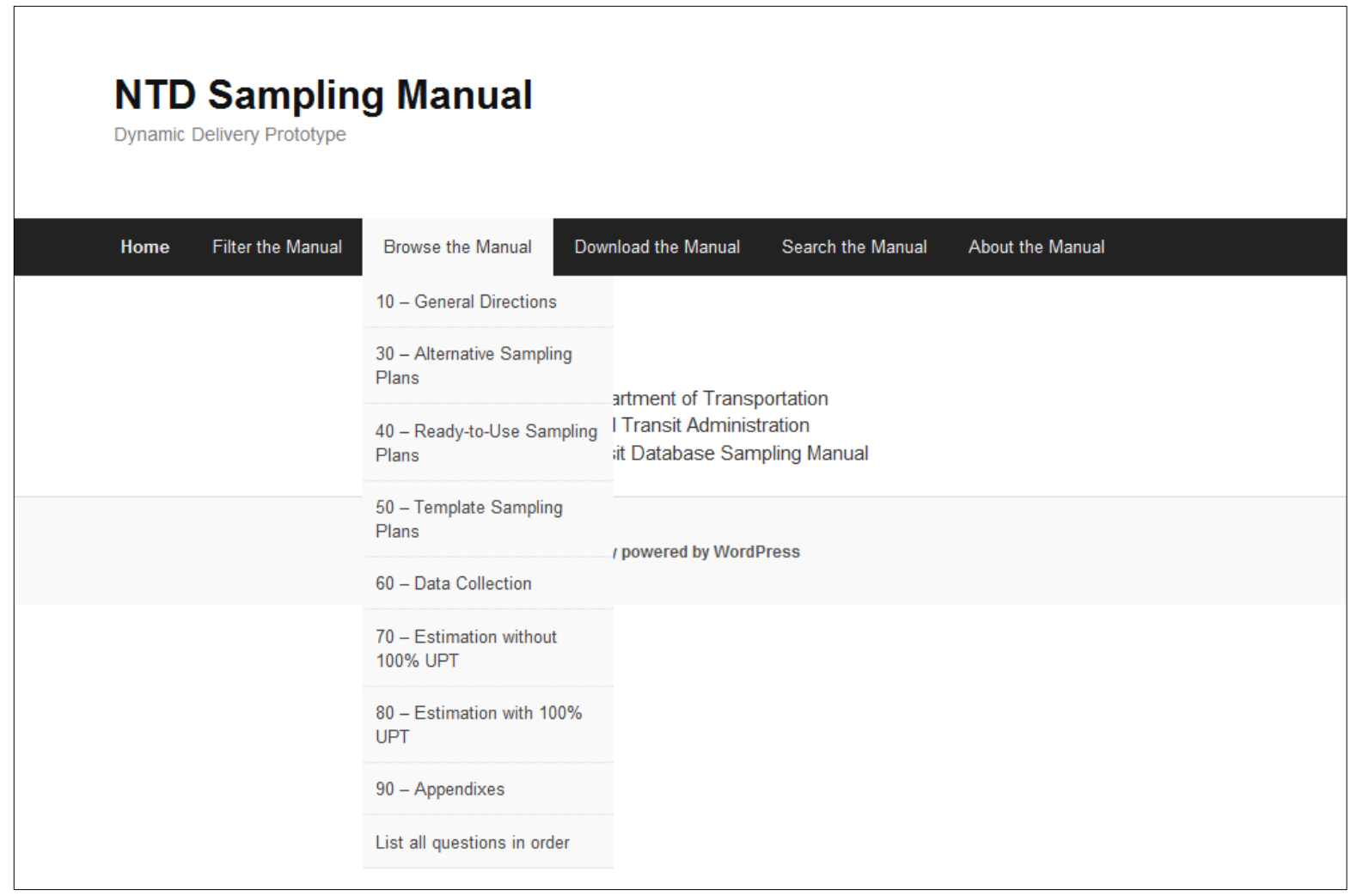

Figure 6. Browsing the Sections 
Subsections. After the drop-down menu appears for the sections, you can move your cursor to a particular section title and hover over it; the subsection numbers and titles will appear to the right of the drop-down menu for sections. Figure 7 illustrates this for section 70 and its subsections. To view the entire content of any subsection, just click its title in the new drop-down menu. The full subsection appears.

\section{NTD Sampling Manual}

Dynamic Delivery Prototype

10 - General Directions

30 - Alternative Sampling

Plans

artment of Transportation

40 - Ready-to-Use Sampling I Transit Administration

Plans

it Database Sampling Manual

50 - Template Sampling

Plans

I powered by WordPress

60 - Data Collection

70 - Estimation without

$100 \%$ UPT

71 - General Directions

80 - Estimation with $100 \%$

UPT

73 - Non-Scheduled

Services

75 - Rail Services

90 - Appendixes

List all questions in order

77 - Bus Services

79 - Estimating Service-

Consumed Data

Figure 7. Browsing the Subsections 
Questions. To get the clickable list of individual pairs of questions/answers for the entire manual for browsing, either directly click the "Browsing the Manual" button at the top or select "List All Questions in Order" in the pull-down menu shown in Figure 6 for sections. The hyperlinked individual pairs of questions/answers appear. Figure 8 shows how the screen appears with questions for the first three sections. The question itself and its full answer appear once the hyperlinked title of this question is clicked.

\title{
NTD Sampling Manual
}

\author{
Dynamic Delivery Prototype
}

\section{Home Filter the Manual Browse the Manual Download the Manual Search the Manual About the Manual}

POSTS

- $\S 10.01$ - What is the purpose of this Sampling Manual?

- $\S 10.03$ - What procedures does this Sampling Manual cover?

- $§ 10.05$ - May I continue using the sampling plans in Circulars $2710.1 \mathrm{~A}$ and $2710.2 \mathrm{~A}$ ?

- $\S 30.01$ - When should I consider using alternative sampling plans?

- $\S 30.03$ - What additional requirements do alternative sampling plans should meet?

- $\$ 41.01$ - Under what conditions may I use ready-to-use sampling plans?

- $§ 41.03$ - If I am using a ready-to-use sampling plan this year, may I use it again for my next report year?

- §41.05 - For what modes are ready-to-use sampling plans available?

- $\$ 41.07$ - What sampling options are available?

Figure 8. Browsing by Questions 
The full manual is available for download both in a Word format and as a PDF file. In addition to the full manual, the following also are available for download:

- Sampling Template without any input data entered

- Sampling Template with input data entered as an example

- Individual appendices of example tables for data collection in Word format

- A random number table as a PDF file

Figure 9 show how the page appears after "Download the Manual" is clicked:

\section{NTD Sampling Manual}

Dynamic Delivery Prototype

- Full Manual

- PDF

- $\mathrm{DOC}$

- Sampling Template

- The NTD Sampling Template Version 2.0

- The NTD Sampling Template Version 2.0 with Sample Data

- Appendices

- Appendix 92 - Distance-Based Survey Sheet

- Appendix 93 - Load-Based Survey Sheet for Demand Response (DR or DT)

- Appendix 94 - Load-Based Survey Sheet for Commuter Vanpool

- Appendix 95 - Load-Based Survey Sheet for Fixed-Route Service

- Appendix 96 - Daily Log for Commuter Vanpool

- Appendix 98 - Table of Random Numbers

Proudly powered by WordPress

Figure 9. Download the Manual 


\section{Search the Manual}

A fourth option to get to the manual is the "Search the Manual" function of the Web tool. The following appears after you click "Search the Manual" at the top:

\section{Type search terms and hit the return key:}

The Web tool delivers every pair of question/answer that contains all search terms you have used for the search. As an illustration, Figure 10 shows the search result from using "certify" as the search term.

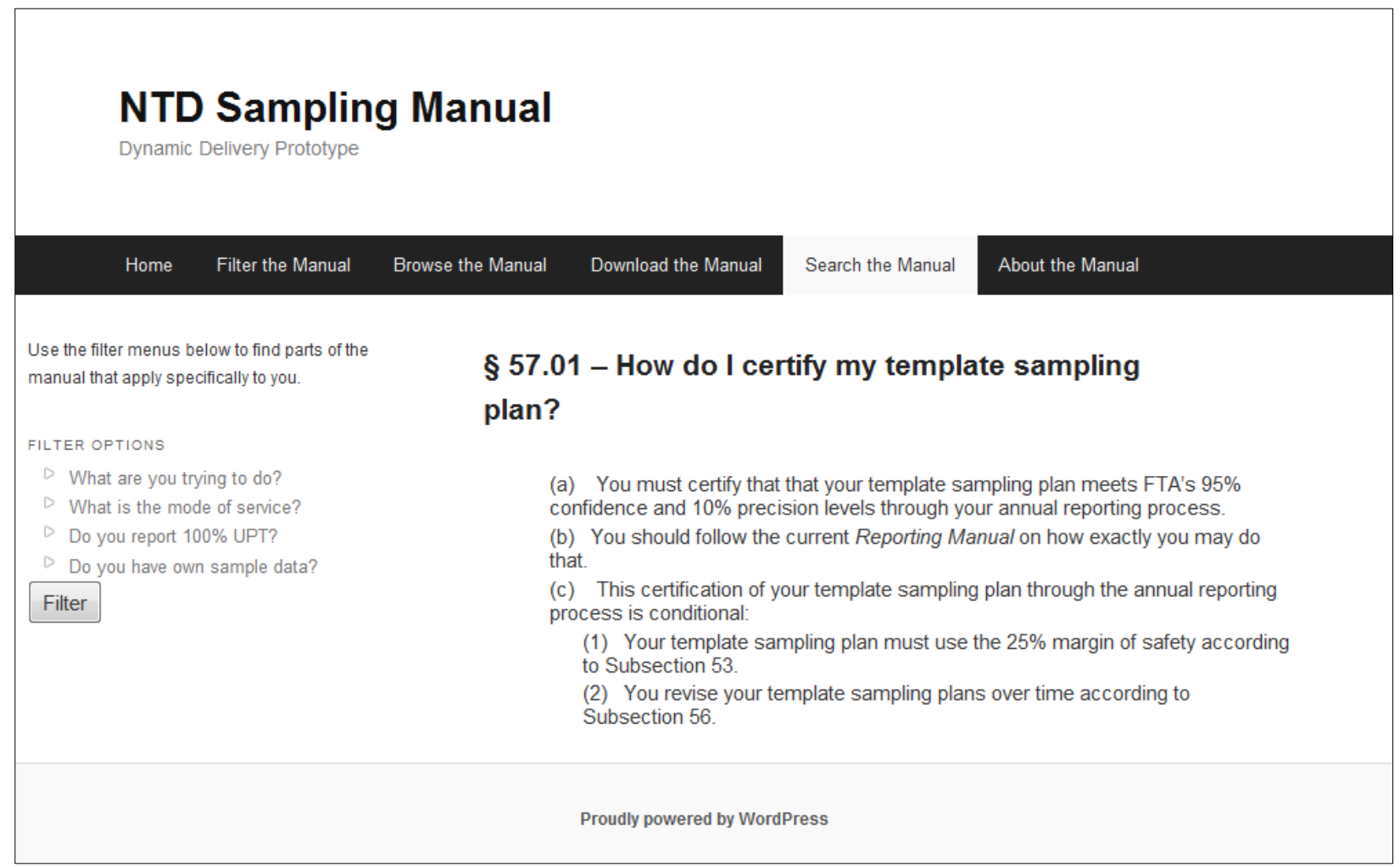

Figure 10. Result of Searching for "Certify" 


\section{Chapter 5 - Conclusions}

\section{Summary}

Enhancements to the Manual

The project has successfully revised the NTD Sampling Manual to incorporate a number of enhancements over the existing version in response to a request by FTA. For example, the new manual adds the requirement for a minimum sample size of 50 for all new sampling plans and for all sample data to be used in developing new sampling plans. Also, the new manual now has a simplified procedure for transit agencies to determine whether they should revise their previously-developed template sampling plans. The most significant improvement is that the new manual includes procedures for transit agencies to develop sampling plans that are similar in structure with those in Circular 2710.1A.

This study also investigated several potential enhancements suggested by FTA that eventually were not incorporated into the new manual. FTA suggested these to reduce the potential negative impact on transit agencies as a result of the recent split of the traditional single mode of bus services into three modes-regular bus, commuter bus, and bus rapid transit. One of these suggested enhancements was to allow transit agencies to estimate and report unlinked passenger trips and passenger miles traveled for these three bus modes combined for a given type of service (directly-operated or purchased). Combined sampling has its disadvantages, but the potential benefits of combined sampling are far less than initially thought. Another suggestion was to waive the newly-added minimum sample size of 50 for certain services, such as single-route services of commuter bus and commuter rail. However, the number of agencies that can benefit from this waiver is likely to be small, and its benefits in terms of the reduction in annual sample size also are small in absolute terms.

\section{Web Tool of a Range of Delivery Options}

More important, researchers for this project successfully developed a Web tool for the new NTD Sampling Manual to be delivered to its users in a range of options. Users can download the entire manual and the Sampling Template and can browse the manual by its sections, subsections, or even individual pairs of questions/answers. They can specify what they need in terms of four profiling questions (e.g., What is your mode of service?) and their various options (e.g., vanpool), and the Web tool can deliver the relevant content of the manual. The delivery options greatly condense and simplify the full manual to many transit agencies and their special guidance needs for obtaining annual totals of unlinked passenger trips and passenger miles traveled, particularly those small- and mid-size agencies that operate only regular bus and demand-response services with small fleets.

\section{Lessons Learned}

As a dynamic content delivery mechanism, the Web tool does not realize all its potential. Dynamic deliveries are not always executed cleanly in some situations. The previous 
version of the manual was not prepared with dynamic delivery in mind. Consequently, some of its individual pairs of questions/answers cover content that includes more than one option of a given profiling question. For example, the guidance for a particular question in the manual may relate to agencies that report $100 \%$ counts of unlinked passenger trips in one part of its answer but to agencies that do not report $100 \%$ counts in another part of its answer. As a result, both parts of the answer would be delivered for either profiling option for the profiling question of whether an agency reports $100 \%$ counts. That is, the delivered content is not clean in that it relates to both profiling options. In many such cases, one pair of question/answer may be broken into two or more pairs. This breaking of existing questions/answers was considered but not adopted for at least two reasons: it will significantly increase the number of questions in the manual, and the resulting manual will look significantly different from the existing version. Neither of these is desirable.

One lesson learned from this shortcoming of the current Web tool is that, for future efforts of developing Internet-based dynamic content delivery tools, the full content document should be prepared in such a way that each of its individual topics does not include content for more than one option for any given profiling question. The challenge is for the resulting document to be coherent among its different sections and individual topics and still to visually be as appealing as a document that is not designed for dynamic delivery. 


\section{References}

Gupta, Pushpa L., and R.D. Gupta. 1987. "Sample Size Determination in Estimating a Covariance Matrix." Computational Statistics and Data Analysis 5: 185-192.

Department of Homeland Security. "DHS Senior Leadership: The First Five Years: 20032008." http://www.d hs.gov/xabout/history/, accessed November 7, 2009.

Kiefer, John J., Richard Martin, and Robert S. Montjoy. 2006. "Incrementalism before the Storm: Network Performance for the Evacuation of New Orleans." Public Administration Review 53(2): 134-139.

Papacostas, S., and D. Prevedouros, D. 2001. Transportation Engineering \& Planning. Upper Saddle River, NJ: Prentice Hall.

Schokkin, J. W. 2007. Emergency Evacuation of the Baltimore City Center in Case of a Chemical Accident. Master's Thesis, University of Twente, Netherlands.

Sterzin, R., and J. Toledo. 2005. "Influencing Factors in Microscopic Traffic Simulation." Presented at the TRB 84th Annual Meeting, January 9-13, 2005, Washington, D.C. 


\section{Appendix - NTD Sampling Manual 2.0}




\begin{tabular}{|c|c|}
\hline & $\begin{array}{c}\text { NATIONAL TRANSIT DATABASE } \\
\text { SAMPLING MANUAL }\end{array}$ \\
\hline & \\
\hline
\end{tabular}

\section{TABLE OF CONTENTS}

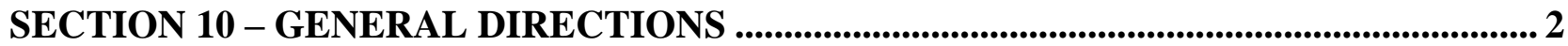

SECTION 30 - ALTERNATIVE SAMPLING PLANS ..................................................... 3

SECTION 40 - READY-TO-USE SAMPLING PLANS ............................................................ 4

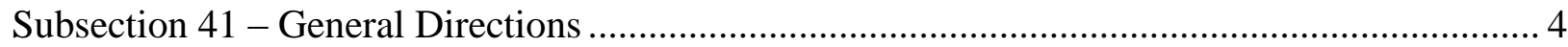

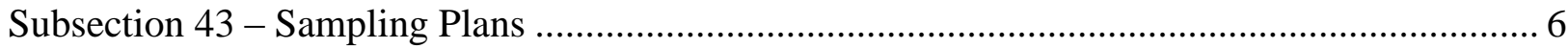

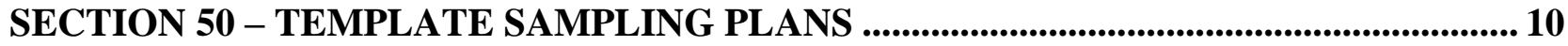

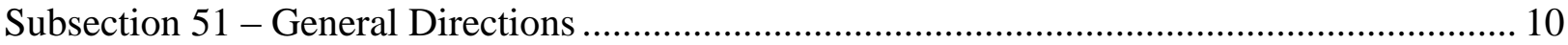

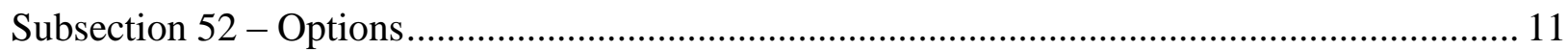

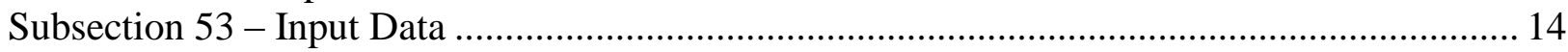

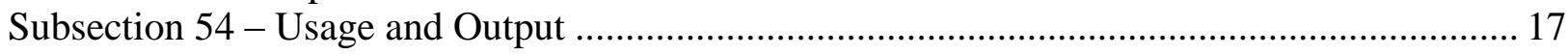

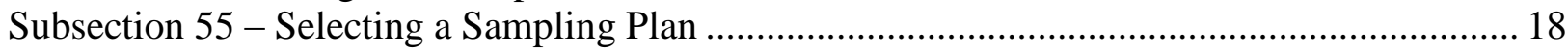

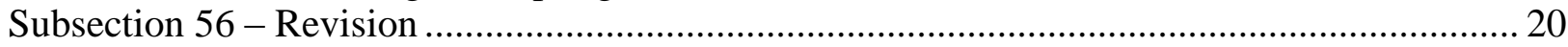

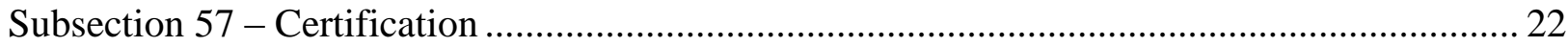

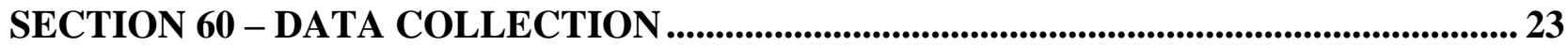

Subsection 61 - General Directions ...................................................................... 23

Subsection 63 - Selecting a Sample at Random .............................................................. 23

Subsection 65 - Collecting Data from the Random Sample ............................................... 26

Subsection 67 - Correcting Errors in the Raw Sample Data .............................................. 33

SECTION 70 - ESTIMATION WITHOUT 100\% UPT ........................................................... 39

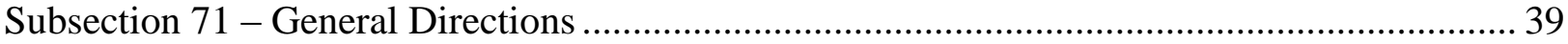

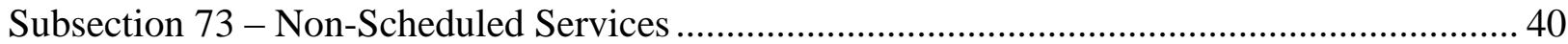

Subsection 75 - Rail Services ............................................................................... 41

Subsection 77 - Bus Services..................................................................................... 43

Subsection 79 - Estimating Service-Consumed Data ....................................................... 45

SECTION 80 - ESTIMATION WITH 100\% UPT ......................................................................... 46

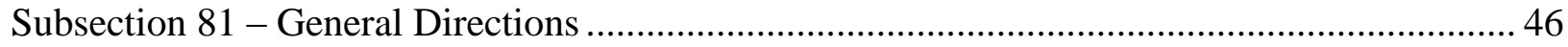

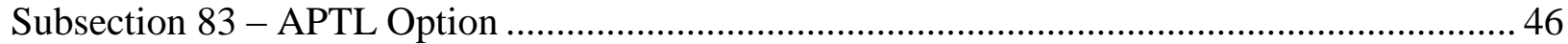

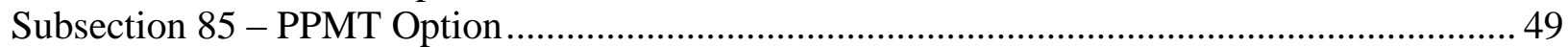

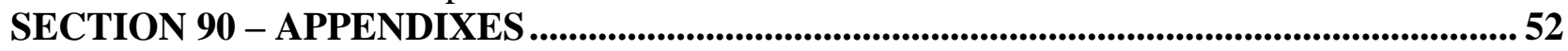

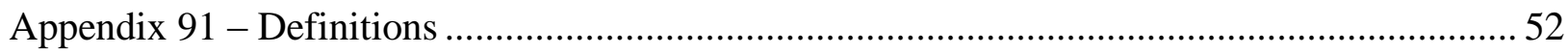

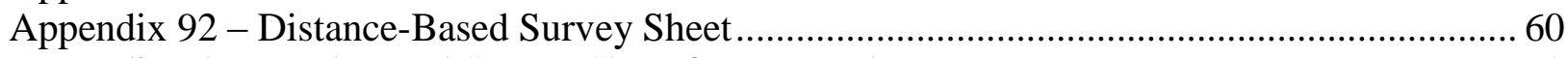

Appendix 93 - Load-Based Survey Sheet for Demand Response (DR or DT) ....................... 62

Appendix 94 - Load-Based Survey Sheet for Commuter Vanpool ........................................... 64 Appendix 95 - Load-Based Survey Sheet for Fixed-Route Service Error! Bookmark not defined. Appendix 96 - Daily Log Sheet for Commuter Vanpool ............... Error! Bookmark not defined. Appendix 97 - Full Table of Contents ............................................. Error! Bookmark not defined. Appendix 98 - Table of Random Numbers ................................. Error! Bookmark not defined. 


\section{SECTION 10 - GENERAL DIRECTIONS}

\section{$\S 10.01 \quad$ What is the purpose of this Sampling Manual?}

(a) It suggests procedures for obtaining annual data on unlinked passenger trips (UPT) and passenger miles traveled (PMT) for the National Transit Database (NTD) through random sampling according to the requirements in the NTD Reporting Manual.

(b) In the event of a conflict between the requirements in the Reporting Manual and this Sampling Manual, the requirements of the Reporting Manual are definitive.

(c) When it is possible to obtain annual UPT and PMT data according to the requirements in the Reporting Manual by using other methods not specified in this Sampling Manual, then you may do so.

\section{$\S 10.03$ What procedures does this Sampling Manual cover?}

(a) Development of sampling plans with two options:

(1) Section 40 on ready-to-use sampling plans.

(2) Section 50 on agency-developed template sampling plans with agency sample data.

(b) Collection of sample data in Section 60.

(c) Estimation of annual service-consumed data with two options:

(1) Both UPT and PMT if you do not report $100 \%$ counts of UPT.

(2) PMT if you report $100 \%$ counts of UPT.

(d) Section 30 specifies several requirements for alternative sampling plans that template sampling plans must also meet.

(e) Figure 10.01 shows how these procedures and options relate to each other.

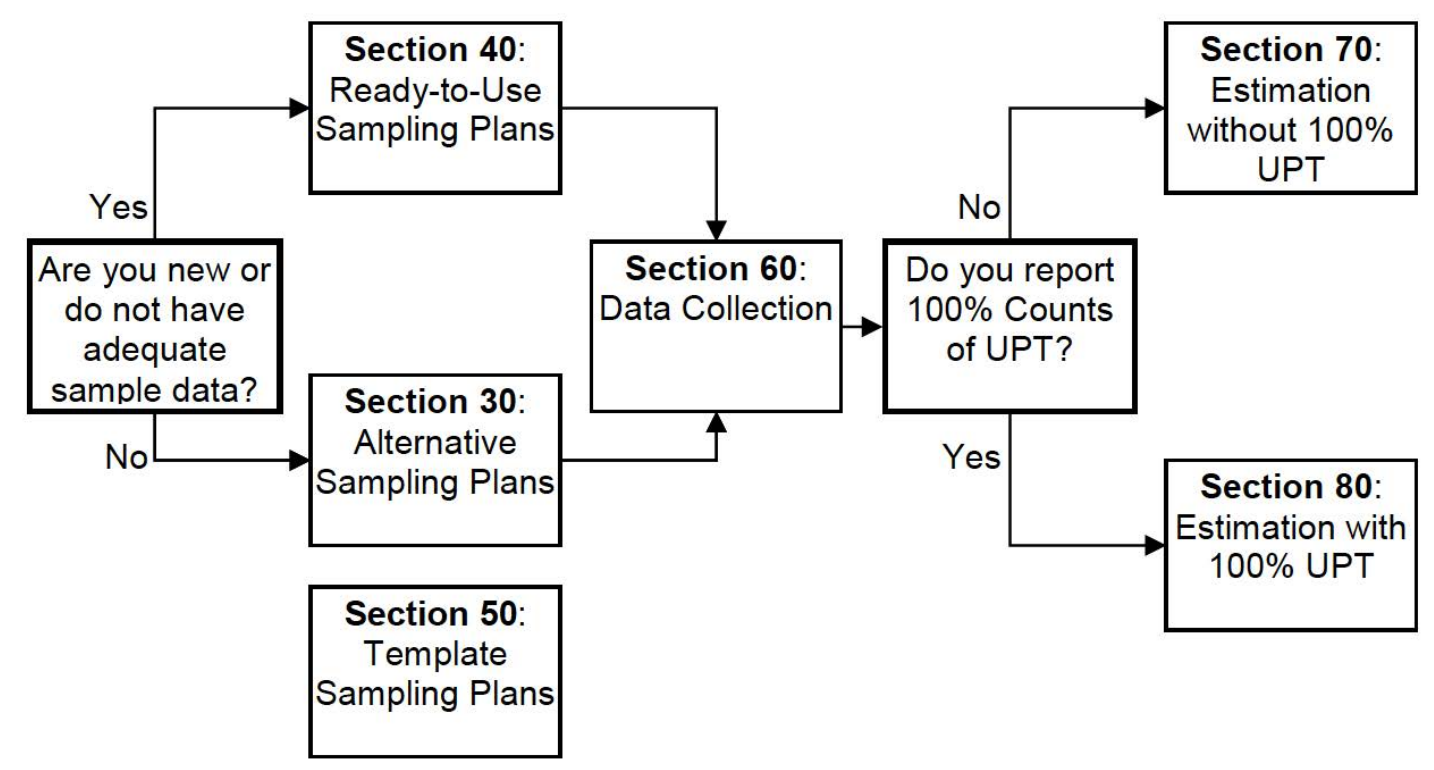

Figure 10.01. Flow Chart of Procedures and Options

$\S 10.05 \quad$ May I continue using the sampling plans in Circulars 2710.1A and 2710.2A?

(a) You may continue using these sampling plans until they are withdrawn. 


\section{SECTION 30 - ALTERNATIVE SAMPLING PLANS}

\section{§ 30.01 When should I consider using alternative sampling plans?}

(a) An alternative sampling plan is equivalent to an alternative sampling technique as defined in the NTD Reporting Manual.

(b) If the sampling options provided in the companion template of this NTD Sampling Manual meet your needs, it is to your advantage to use template sampling plans.

(c) You should consider using alternative sampling plans when the companion template does not cover the best option for your specific situation. The companion template provides many options for you to explore and consider for the vast majority of possible situations, but it may not cover the best option for all situations.

\section{§30.03 What additional requirements should alternative sampling plans meet?}

(a) Alternative sampling plans must meet FTA's $95 \%$ confidence and $10 \%$ precision levels.

(b) In addition to these basic statistical requirements, alternative sampling plans should also meet the following requirements:

(1) They are based on the conditions of your service. That means that they should be developed with sample data collected from a random sample of your service.

(2) The sample data used for developing alternative sampling plans have a minimum sample size of 50 service units.

(3) They contain a margin of safety of at least $25 \%$. That is, the final sample size of an alternative sampling plan is at least $25 \%$ greater than what you would get from using the sample data directly.

(4) The final sample size of any alternative sampling plan is at least 50 .

(c) Template sampling plans also should meet the additional requirements in (b) as specified in Section 50. 


\section{SECTION 40 - READY-TO-USE SAMPLING PLANS}

\section{Subsection 41 - General Directions}

\section{\$ 41.01 Under what conditions may I use ready-to-use sampling plans?}

(a) Small Systems - If you operate no more than 30 vehicles operated in maximum service for all modes combined and if you choose to sample for determining your annual serviceconsumed data for any of the modes you operate.

(b) New Mode - If you will be sampling and reporting for the first time this current report year for a particular mode that you do not already operate. For example, you would meet this condition if you will add light rail (LR) service this year, but you have not operated this service previously, or

(c) New Type of Service - If you will be sampling and reporting this current report year for a particular type of service for the first time. For example, you would meet this condition if you previously directly operated all of your bus (MB) service, but will contract out part or all of that service to a private entity for this year, or

(d) Major Changes of Service - You have made major changes to your service since your last sampling year, or

(e) No Sample Data - If you have reported your service to the NTD before through random sampling, but no longer have the original sample data, or

(f) Sample Data Not Reliable - If you have reported your service to the NTD before through random sampling, but the sample data are found to be not reliable, or

(g) Sample Data Not Enough - if you have reported your service to the NTD before through random sampling, but the sample size is smaller than 50 service units.

$\S 41.03$ If I am using a ready-to-use sampling plan this year, may I use it again for my next report year?

(a) You should not use it again if your next report year is your mandatory sampling year. After you have collected the sample data from this year, you should develop a template sampling plan with that sample data for your next report year.

(b) You may use it again for the next report year if that year is not your mandatory sampling year.

(c) You may use it while you operate no more than 30 vehicles operated in maximum service for all modes combined.

\section{\$ 41.05 For what modes are ready-to-use sampling plans available?}

(a) For non-scheduled services, they are available for demand response services (DR and DT) and commuter vanpool. You should not use the ready-to-use sampling plans for commuter vanpool if your vanpool service does not serve commuters exclusively.

(b) For scheduled services, they are available for bus services (bus (MB), commuter bus (CB), bus rapid transit (RB), and trolleybus (TB)), commuter rail (CR), and other rail modes. 


\section{§41.07 What sampling options are available?}

(a) The available sampling options vary in:

(1) the unit of sampling and measurement,

(2) sampling structure,

(3) efficiency options, and

(4) sampling frequency.

(b) The particular unit of sampling and measurement used in these ready-to-use sampling plans varies by mode and whether the service is scheduled (Table 41.01).

(1) For non-scheduled services, the unit is in vehicle days.

(2) For bus services (MB, CB, RB, and TB), separate sampling plans are available with units in one-way trips and in round trips.

(3) For commuter rail, the unit is in one-way car trips.

(4) For other rail modes, separate sampling plans are available in units of one-way car trips and one-way train trips.

Table 41.01. Options for Unit of Sampling and Measurement

\begin{tabular}{|l|l|l|}
\hline Service & Mode & Units of Sampling and Measurement \\
\hline \multirow{3}{*}{ Non-Scheduled } & Demand Response (DR, DT) & Vehicle days \\
\cline { 2 - 3 } & Commuter Vanpool & Vehicle days \\
\hline \multirow{3}{*}{ Scheduled } & Bus (MB, CB, RB, TB) & One-way trips, round trips \\
\cline { 2 - 3 } & Commuter Rail (CR) & One-way car trips \\
\cline { 2 - 3 } & Other Rail Modes & One-way car trips, one-way train trips \\
\hline
\end{tabular}

(c) Two options are provided for sampling structure-period-based and interval-based. Interval-based sampling plans are available for bus services only.

(d) Three efficiency options are provided:

(1) Base Option - you must estimate both UPT and PMT through random sampling.

(2) APTL Option - you must report a 100\% count of UPT, estimate the average passenger trip length (APTL) through random sampling, and obtain annual PMT by multiplying the $100 \%$ UPT with the estimated APTL.

(3) Grouping Option - you must divide your bus routes into two groups by route length. The grouping option is available for period-based sampling plans only.

(e) Three frequency options are provided for period-based sampling plans-quarterly, monthly, or weekly. You may choose whichever of these options is best suited for your agency. One factor to consider is that the annual realized sample size may be larger at a lower sampling frequency due to rounding. Another factor is that a lower sampling frequency means a larger annual number of acts for random sampling.

(f) Up to six frequency options are provided for interval-based sampling plans - every day, every $2^{\text {nd }}$ day, every $3^{\text {rd }}$ day, every $4^{\text {th }}$ day, every $5^{\text {th }}$ day, and every $6^{\text {th }}$ day. Three sets of these sampling plans are available for 7-day weekly service, 6-day weekly service, and 5day weekly service, respectively. The sampling plans for 7-day weekly service and the base option are similar to those in Circular 2710.1A. 


\section{Subsection 43 - Sampling Plans}

\$ 43.01 What period-based sampling plans are available for non-scheduled services?

(a) Table 43.01 shows the sampling plans available for demand response services (DR and DT) and commuter vanpool, respectively.

(b) Sample size is stated in the number of vehicle days.

(c) Separate sampling plans are available for the base option and the APTL option.

(d) Sample size is shown for the entire year and for the relevant period for each frequency.

Table 43.01. Period-Based Ready-to-Use Sampling Plans for Non-Scheduled Services

\begin{tabular}{|c|c|c|c|c|c|}
\hline \multirow[b]{2}{*}{$\begin{array}{l}\text { Sampling } \\
\text { Frequency }\end{array}$} & \multirow[b]{2}{*}{ Sample Size } & \multicolumn{2}{|c|}{ Demand Response } & \multicolumn{2}{|c|}{ Commuter Vanpool } \\
\hline & & $\begin{array}{c}\text { Reporting } \\
\text { 100\% UPT } \\
\text { (APTL Option) }\end{array}$ & $\begin{array}{c}\text { Not Reporting } \\
100 \% \text { UPT } \\
\text { (Base Option) }\end{array}$ & $\begin{array}{c}\text { Reporting } \\
\text { 100\% UPT } \\
\text { (APTL Option) } \\
\end{array}$ & $\begin{array}{l}\text { Not Reporting } \\
100 \% \text { UPT } \\
\text { (Base Option) }\end{array}$ \\
\hline \multirow{2}{*}{ Quarterly } & Vehicle Days for a Quarter & 13 & 22 & 31 & 45 \\
\hline & Total Sample Size for Year & 52 & 88 & 124 & 180 \\
\hline \multirow{2}{*}{ Monthly } & Vehicle Days for a Month & 5 & 8 & 10 & 15 \\
\hline & Total Sample Size for Year & 60 & 96 & 120 & 180 \\
\hline \multirow{2}{*}{ Weekly } & Vehicle Days for a Week & 1 & 2 & 2 & 4 \\
\hline & Total Sample Size for Year & 52 & 104 & 104 & 208 \\
\hline
\end{tabular}

\$ 43.03 What period-based ready-to-use sampling plans are available for bus services?

(a) Table 43.03 shows the available ready-to-use sampling plans for bus services, including bus (MB), commuter bus (CB), bus rapid transit (RB), and trolleybus (TB).

Table 43.03. Period-Based Ready-to-Use Sampling Plans for Bus Services

\begin{tabular}{|c|c|c|c|c|c|c|c|}
\hline \multirow{4}{*}{$\begin{array}{l}\text { Sampling } \\
\text { Frequency }\end{array}$} & \multirow{4}{*}{$\begin{array}{l}\text { Sample Size for Period and } \\
\text { Year }\end{array}$} & \multicolumn{3}{|c|}{ One-Way Trips } & \multicolumn{3}{|c|}{ Round Trips } \\
\hline & & \multicolumn{2}{|c|}{$\begin{array}{l}\text { Reporting } 100 \% \text { UPT } \\
\text { (APTL Option) }\end{array}$} & \multirow{2}{*}{$\begin{array}{c}\text { Not } \\
\text { Reporting } \\
100 \% \text { UPT } \\
\text { (Base } \\
\text { Option) }\end{array}$} & \multicolumn{2}{|c|}{$\begin{array}{l}\text { Reporting } 100 \% \text { UPT } \\
\text { (APTL Option) }\end{array}$} & \multirow{2}{*}{$\begin{array}{c}\text { Not } \\
\text { Reporting } \\
100 \% \text { UPT } \\
\text { (Base } \\
\text { Option) } \\
\end{array}$} \\
\hline & & $\begin{array}{l}\text { With Route } \\
\text { Grouping }\end{array}$ & $\begin{array}{l}\text { Without } \\
\text { Route } \\
\text { Grouping }\end{array}$ & & $\begin{array}{l}\text { With Route } \\
\text { Grouping }\end{array}$ & $\begin{array}{l}\text { Without } \\
\text { Route } \\
\text { Grouping }\end{array}$ & \\
\hline & & (1) & (2) & (3) & (4) & (5) & (6) \\
\hline \multirow{2}{*}{ Quarterly } & Trips for a Quarter & 52 & 78 & 138 & 39 & 59 & 103 \\
\hline & Total Sample Size for Year & 208 & 312 & 552 & 156 & 236 & 412 \\
\hline \multirow{2}{*}{ Monthly } & Trips for a Month & 18 & 27 & 46 & 13 & 20 & 35 \\
\hline & Total Sample Size for Year & 216 & 324 & 552 & 156 & 240 & 420 \\
\hline \multirow{2}{*}{ Weekly } & Trips for a Week & 4 & 6 & 11 & 3 & 5 & 8 \\
\hline & Total Sample Size for Year & 208 & 312 & 572 & 156 & 260 & 416 \\
\hline
\end{tabular}


(b) Sampling plans are provided separately for one-way trips and round trips.

(c) The number of one-way trips in a sampling plan based on round trips is about $50 \%$ greater than the number of one-way trips in a sampling plan based on one-way trips. A sampling plan based on round trips requires a larger number of one-way trips because the pair of one-way trips making up a round trip are not selected randomly or independently.

(d) If you choose the base option:

(1) use column (3) to find the sample size in one-way trips, and

(2) use column (6) to find the sample size in round trips.

(e) If you choose the APTL option, you may choose one of two options:

(1) With Route Grouping - Use column (1) to find the sample size in one-way trips and column (4) to find the sample size in round trips. In using this option, you must divide your routes into two groups on the basis of route length and do sampling and estimation separately for each group. For example, if you operate 10 routes, put the 5 routes with the shortest route distances in the group of short routes and the other 5 routes in the group of long routes.

(2) Without Route Grouping - If you prefer not to deal with grouping your routes, use column (2) to find the sample size in one-way trips and use column (5) to find the sample size in round trips.

\section{$\S$ 43.05 How does grouping of my bus routes affect sampling and estimation procedures?}

(a) You must do your sampling and estimation separately for individual route groups. For details, refer to:

(1) Subsection 63 on sampling.

(2) Section 70 on estimation without $100 \%$ UPT.

(3) Section 80 on estimation with $100 \%$ UPT.

\section{\$ 43.07 What period-based sampling plans are available for commuter rail?}

(a) Table 43.05 shows the available ready-to-use sampling plans for commuter rail (CR).

(b) Sample size is in one-way car trips.

(c) Separate sampling plans are available for the base option and the APTL option.

Table 43.05. Period-Based Ready-to-Use Sampling Plans for Commuter Rail (CR)

\begin{tabular}{|l|l|c|c|}
\hline $\begin{array}{l}\text { Sampling } \\
\text { Frequency }\end{array}$ & Sample Size for Period and Year & $\begin{array}{c}\text { Reporting } \\
\text { 100\% UPT } \\
\text { (APTL Option) }\end{array}$ & $\begin{array}{c}\text { Not Reporting } \\
100 \% \text { UPT } \\
\text { (Base Option) }\end{array}$ \\
\hline \multirow{2}{*}{ Quarterly } & One-Way Car Trips for a Quarter & 13 & 80 \\
\cline { 2 - 4 } & Total Sample Size for Year & 52 & 320 \\
\hline \multirow{2}{*}{ Monthly } & One-Way Car Trips for a Month & 5 & 27 \\
\cline { 2 - 4 } & Total Sample Size for Year & 60 & 324 \\
\hline \multirow{2}{*}{ Weekly } & One-Way Car Trips for a Week & 1 & 7 \\
\cline { 2 - 4 } & Total Sample Size for Year & 52 & 364 \\
\hline
\end{tabular}




\section{§43.09 What period-based sampling plans are available for other rail modes?}

(a) Table 43.07 shows the available ready-to-use sampling plans for other rail modes, including light rail (LR), heavy rail (HR), monorail and automated guideway (MG).

(b) Separate sampling plans are available for one-way train trips and one-way car trips.

(c) Separate sampling plans are available for the base option and for the APTL option.

Table 43.07. Period-Based Ready-to-Use Sampling Plans for Other Rail Modes

\begin{tabular}{|c|c|c|c|c|c|}
\hline \multirow[b]{2}{*}{$\begin{array}{l}\text { Sampling } \\
\text { Frequency }\end{array}$} & \multirow[b]{2}{*}{$\begin{array}{l}\text { Sample Size for Period } \\
\text { and Year }\end{array}$} & \multicolumn{2}{|c|}{ One-Way Train Trips } & \multicolumn{2}{|c|}{ One-Way Car Trips } \\
\hline & & $\begin{array}{c}\text { Reporting } \\
\text { 100\% UPT } \\
\text { (APTL Option) }\end{array}$ & $\begin{array}{l}\text { Not Reporting } \\
100 \% \text { UPT } \\
\text { (Base Option) }\end{array}$ & $\begin{array}{c}\text { Reporting } \\
\text { 100\% UPT } \\
\text { (APTL Option) }\end{array}$ & $\begin{array}{l}\text { Not Reporting } \\
100 \% \text { UPT } \\
\text { (Base Option) }\end{array}$ \\
\hline \multirow{2}{*}{ Quarterly } & Trips for a Quarter & 13 & 45 & 13 & 72 \\
\hline & Total Sample Size for Year & 52 & 180 & 52 & 288 \\
\hline \multirow{2}{*}{ Monthly } & Trips for a Month & 5 & 15 & 5 & 24 \\
\hline & Total Sample Size for Year & 60 & 180 & 60 & 288 \\
\hline \multirow{2}{*}{ Weekly } & Trips for a Week & 1 & 4 & 1 & 6 \\
\hline & Total Sample Size for Year & 52 & 208 & 52 & 288 \\
\hline
\end{tabular}

\section{$\S$ 43.11 What interval-based ready-to-use sampling plans are available for bus} services?

(a) Table 43.09 shows the available ready-to-use sampling plans for bus services, including bus (MB), commuter bus (CB), bus rapid transit (RB), and trolleybus (TB).

(b) Separate sampling plans are available for the base option and the APTL option.

(c) In determining whether using interval-based sampling plans, you should consider the tradeoff between at least two factors:

(1) the advantage of using a sampling plan that you have been using for years, and

(2) the potentially lost opportunity of a lower sample size.

(d) You should first determine the number of weekly operating days for your next sampling year, and choose the set of sampling plans accordingly. For example, if you will be operating 7 days a week, you should not consider any of the sets for the other weekly operating patterns.

(e) You may choose any of the above plan numbers that correspond to the weekly operating pattern of your service. 
Table 43.09. Interval-Based Ready-to-Use Sampling Plans for Bus Services

\begin{tabular}{|c|c|c|c|c|c|c|c|}
\hline \multirow[b]{2}{*}{$\begin{array}{l}\text { Plan } \\
\text { Number }\end{array}$} & \multirow[b]{2}{*}{$\begin{array}{l}\text { Frequency of } \\
\text { Sampling }\end{array}$} & \multicolumn{2}{|c|}{$\begin{array}{c}\text { Operating } 7 \text { Days a } \\
\text { Week }\end{array}$} & \multicolumn{2}{|c|}{$\begin{array}{c}\text { Operating } 6 \text { Days a } \\
\text { Week }\end{array}$} & \multicolumn{2}{|c|}{$\begin{array}{c}\text { Operating } 5 \text { Days a } \\
\text { Week }\end{array}$} \\
\hline & & $\begin{array}{c}\text { Daily One- } \\
\text { Way Bus } \\
\text { Trips }\end{array}$ & $\begin{array}{c}\text { Total } \\
\text { Sample } \\
\text { Size for } \\
\text { Year }\end{array}$ & $\begin{array}{c}\text { Daily One- } \\
\text { Way Bus } \\
\text { Trips }\end{array}$ & $\begin{array}{c}\text { Total } \\
\text { Sample } \\
\text { Size for } \\
\text { Year }\end{array}$ & $\begin{array}{c}\text { Daily One- } \\
\text { Way Bus } \\
\text { Trips }\end{array}$ & $\begin{array}{l}\text { Total } \\
\text { Sample } \\
\text { Size for } \\
\text { Year }\end{array}$ \\
\hline \multicolumn{8}{|c|}{ Base Option } \\
\hline 1 & Every Day & 2 & 730 & 2 & 624 & 2 & 520 \\
\hline 2 & Every $2^{\text {nd }}$ Day & 3 & 549 & 3 & 468 & 4 & 520 \\
\hline 3 & Every $3^{\text {rd }}$ Day & 5 & 610 & 6 & 624 & 7 & 609 \\
\hline 4 & Every $4^{\text {th }}$ Day & 7 & 644 & 9 & 702 & 12 & 780 \\
\hline 5 & Every $5^{\text {th }}$ Day & 10 & 730 & 13 & 819 & & \\
\hline 6 & Every $6^{\text {th }}$ Day & 15 & 915 & & & & \\
\hline \multicolumn{8}{|c|}{ APTL Option } \\
\hline 1 & Every Day & 1 & 365 & 1 & 312 & 1 & 260 \\
\hline 2 & Every $2^{\text {nd }}$ Day & 2 & 366 & 2 & 312 & 3 & 390 \\
\hline 3 & Every $3^{\text {rd }}$ Day & 3 & 366 & 4 & 416 & 5 & 435 \\
\hline 4 & Every $4^{\text {th }}$ Day & 4 & 368 & 6 & 468 & 8 & 520 \\
\hline 5 & Every $5^{\text {th }}$ Day & 6 & 438 & 8 & 504 & & \\
\hline 6 & Every $6^{\text {th }}$ Day & 10 & 610 & & & & \\
\hline
\end{tabular}




\section{SECTION 50 - TEMPLATE SAMPLING PLANS}

\section{Subsection 51 - General Directions}

\section{§ 51.01 Under what conditions should I develop a template sampling plan for next year?}

(a) If you have previously been using a ready-to-use sampling plan and the next report year is your mandatory sampling year;

(b) If you have previously been using a sampling plan, but it is not based on the conditions of your service;

(c) If you have previously been using a template sampling plan, but you must revise it for your next sampling year according to Subsection 56; or

(d) If you previously have been using an alternative sampling plan, but would like to:

(1) improve your sampling efficiency,

(2) change the unit of sampling and measurement,

(3) change the sampling structure, or

(4) use a different sampling frequency.

\section{$\S 51.02$ Should I consider template sampling plans if I am a small system and want to sample?}

(a) You do not have to consider template sampling plans. You may continue using ready-touse sampling plans while you continue operate no more than 30 vehicles operated in maximum service for all modes combined.

(b) It is likely to be in your interest to consider template sampling plans.

(c) If the sample size requirement is considerably smaller for template sampling plans than for ready-to-use sampling plans, the benefit is likely to be much greater than your cost from considering template sampling plans.

\section{\$1.03 Does this Manual have a companion tool that I may use?}

(a) Yes, this companion tool is an Excel template, "The NTD Sampling Template.xlsm."

(b) It is specifically designed for you to develop template sampling plans.

(c) You may download a copy of this template from the NTD homepage at www.ntdprogram.gov.

\section{$\$ 51.05 \quad W h a t$ is the scope of this template?}

(a) You may use it for any mode of transit service, such as bus (MB), that is explicitly recognized by the NTD.

(b) You may use it for any type of service, such as directly operated (DO), that is explicitly recognized by the NTD.

(c) You may use it with sample data in any unit of sampling and measurement.

\section{\$ 51.07 Do I need to have knowledge of statistics to use this template?}

(a) You do not need any knowledge of statistics to use this template.

(b) You must follow the steps exactly as described. 


\section{Subsection 52 - Options}

\$ 52.01 What types of options does this template provide in its sampling plans?

(a) Unit of sampling and measurement - the unit of service at which you do sampling, data collection, and estimation.

(b) Sampling structure - period-based sampling or interval-based sampling.

(c) Sampling frequency - how frequently you do sampling.

\section{\$ 52.03 Must I pick a specific unit of sampling and measurement to use this template?}

(a) You must pick a specific unit of sampling and measurement before you start using this template. The type of sample data you have available will often dictate your choice of a unit for sampling and measurement.

(1) For non-scheduled services, such as demand response (DR and DT) or vanpool (VP), the unit of sampling and measurement is typically the vehicle day.

(2) For bus services, including bus (MB), commuter bus (CB), bus rapid transit (RB), and trolley bus (TB), you must decide if the unit will be a one-way bus trip or a round-trip bus trip. It is a common practice to use a one-way bus trip as the unit of sampling and measurement.

(3) For rail services, you must decide if the unit will be a one-way car trip, a round-trip car trip, a one-way train trip, or a round-trip train trip. It is a common practice to use one-way passenger car trip as the unit of sampling and measurement.

(b) The sample data you enter into this template will all be in this unit.

(c) The resulting sampling plans from this template will also be in this unit.

(d) The expansion factors you are going to use to expand your sample will also be in this unit if you are going to estimate both UPT and PMT with your sample data.

\section{\$ 52.04 What options does this template provide on sampling structure?}

(a) Period-based sampling plans - you sample the same number of service units for each period (e.g., monthly).

(b) Interval-based sampling plans - you sample the same number of service units one each sample day, and the interval between the sample days is constant.

(c) In determining whether using interval-based sampling plans, you should consider their advantages and disadvantages:

(1) the advantage of using a sampling plan that you have been using for years,

(2) the advantage of using regular sample days throughout a year,

(3) the disadvantage of a large number of times you need to sample during a year,

(4) the disadvantage of not being able to consider the PPMT and grouping options, and

(5) the disadvantage of a likely larger annual sample size due to rounding in determining the daily sample size.

\section{§52.05 What options does this template provide on sampling frequency?}

(a) This template provides you with three frequency options for period-based sampling quarterly, monthly, and weekly. For example, if weekly sampling requires 4 one-way bus trips per week, you must select at least 4 one-way bus trips at random from the full list of one-way bus trips that you expect to operate during a particular week.

(b) This template provides you with up to 6 frequency options for interval-based sampling: 
(1) 7-day weekly service - every day, every $2^{\text {nd }}$ day, every $3^{\text {rd }}$ day, every $4^{\text {th }}$ day, every $5^{\text {th }}$ day, and every $6^{\text {th }}$ day.

(2) 6-day weekly service - every day, every $2^{\text {nd }}$ day, every $3^{\text {rd }}$ day, every $4^{\text {th }}$ day, and every $5^{\text {th }}$ day.

(3) 5-day weekly service - every day, every $2^{\text {nd }}$ day, every $3^{\text {rd }}$ day, and every $4^{\text {th }}$ day.

(c) You may choose whichever frequency option is best for your agency.

\section{$\$$ 52.07 What should I consider in choosing a sampling frequency?}

(a) Period-based sampling plans:

(1) Your cycle of minor schedule changes during a year. For example, quarterly sampling may be appropriate if you routinely adjust your schedule every three months.

(2) The scale of your operation and the method you will be using to select a sample. For example, it may become difficult to select a sample for an entire quarter if the number of service units involved in a quarter is too large for a spreadsheet to handle.

(3) The realized annual sample size. The realized annual sample size can be larger than the initial annual sample size due to rounding in allocating the initial annual sample to each frequency level. This is particularly true for weekly sampling when the initial annual sample size is relatively small. For example, if the initial annual sample size is 55 and you choose weekly sampling, the weekly sample size would be 2 and the realized annual sample size would be 104 .

(b) Interval-based sampling plans:

(1) Your staffing needs for data collection.

(2) The number of times you need to do sampling. The number of times is larger with plans of higher frequency (e.g., every vs. every $2^{\text {nd }}$ day).

(3) The annual total sample size. The degree of rounding is higher with plans of higher frequency.

\section{\$ 52.09 What efficiency options does the template include?}

(a) The template includes a number of options for you to consider in improving your sampling efficiency:

(1) The base option.

(2) The APTL option.

(3) The PPMT option.

(4) The grouping option.

(b) You may use the base option if you are going to estimate both UPT and PMT (i.e., you will not be using $100 \%$ counts for either UPT or PMT), and

(c) Any of the other three options has the potential to improve your sampling efficiency over the base option:

(1) Sampling plans under the APTL option are based on how APTL in absolute terms varies across your service units.

(2) Sampling plans under the PPMT option are based on how APTL relative to average route length varies across your service units.

(3) Sampling plans under the grouping option are based on the idea that relevant statistical variation may be smaller across service units within sub-segments of your service than across all service units of your entire service. 
(d) Additional options become available when service grouping is combined with each of the other three efficiency options.

\section{\$ 52.11 Which of these efficiency options are available to me?}

(a) All four efficiency options are available for period-based sampling. Only the first two are available for interval-based sampling.

(b) The base option is available to you if you have data on PMT from your sample. But it is not recommended in most cases if a $100 \%$ count of annual UPT is available and reliable.

(c) The APTL option is available to you if you meet the following two criteria:

(1) you are going to report a $100 \%$ count of annual UPT, and

(2) you have data on both UPT and PMT from your sample data.

(d) The PPMT option is available to you if you meet all of the following criteria:

(1) you are going to report a $100 \%$ count of annual UPT,

(2) you have data on both UPT and PMT from your sample data,

(3) your service is a fixed-route service,

(4) you have your $100 \%$ count of annual UPT for each route, and

(5) you have data on annual total vehicle revenue miles and annual total vehicle revenue one-way trips for each route.

(e) The grouping option is available to you as long as you have some basis for dividing your service into two or more groups.

\section{\$ 52.13 How do I divide my scheduled service if I want to consider the grouping option?}

(a) Grouping is usually by route when there is a small number of routes or by groups of routes that are similar.

(b) You are going to determine the similarity of service units on your expectations from your prior knowledge of your service.

(c) The most efficient grouping depends on other efficiency options you consider:

(1) If your sampling plan follows the base option, define groups so that within each group, they are similar with respect to PMT per service unit. For example, you may group heavily used long routes separately from lightly used shorter routes.

(2) If your sampling plan follows the APTL option, define your groups so that within each group they have similar APTL. For example, if your express route customers travel particularly long distances, you may separate your express and local services as two groups. As another example, if some of your local routes are particularly longer than the other local routes, you may further divide your local service into two groups.

(3) If your sampling plan follows the PPMT option, define your groups so that the routes within each group are similar with respect to the fraction of a route's length that passengers typically ride. For example, if on some routes passengers tend to ride most of length of the route (as is the case for some express routes) while on other routes passengers tend to ride for only a small part of the route's full length, separate those routes into different groups. Consider another example. If a system has a mix of radial routes (routes with one end in the downtown), diagonal routes (routes that pass through the downtown), and crosstown routes (routes that don't pass through the downtown), the average fraction of route length that passengers ride tends to be smaller on the diagonal and crosstown routes and longer on the radial routes. In this case, radial routes could be grouped separately from diagonal and crosstown routes. 


\section{\$ 52.15 How do I group my vanpool service if I serve commuters only?}

(a) You should base the grouping of your service on the registered round-trip distance of each vanpool.

(b) In most cases you will only need two groups with the longer routes in one group and the shorter routes in another group.

\section{\$ 52.17 What should I consider in general when I consider grouping my service?}

(a) Strike a balance between the number of groups and the potential reduction in necessary sample size. In most cases, you will only want to deal with two or three groups.

(b) You may try different ways of grouping your service and choose one particular grouping based on its efficiency improvement and your administrative convenience.

(c) The grouping option may be used for administrative convenience alone:

(1) You may treat your different contractors of service for a given mode as separate groups when each is responsible for sampling and collecting data.

(2) You may treat your operational divisions as separate groups if each is responsible for sampling and collecting data.

(3) You may treat your commuter vanpool separately from your other vanpool services.

\section{Subsection 53 - Input Data}

\section{\$ 53.01 What data must I have to use this template?}

(a) You must have a set of sample data collected recently from your service for the mode and type of service for which you would like to have a new template sampling plan.

(1) The sample data must be at the unit of sampling and measurement you have chosen for your sampling plan. For example, if your sampling plan is in one-way bus trips, the sample data should show the UPT and PMT and other identification information for each one-way bus trip in your sample.

(2) Follow the instructions in the template for details on data items and format required and how they should be entered into the template.

(b) You should use your NTD sample if it is available and you have not made major changes to your service since the data were collected.

(c) Otherwise, you may use data that have been collected for non-NTD purposes:

(1) One good source of non-NTD sample data for scheduled services is a comprehensive operational analysis (COA). Typically, all trips or a large portion of all trips on your schedule are checked by ride-checkers or by APCs during a concentrated period.

(2) Make sure that the non-NTD source of sample data has the required information for using this template. For example, between-stop distances often are not collected during COA ride checks, and the resulting ride check data would not be useful if you do not have predetermined between-stop distances for your entire service.

(d) The sample size of the sample data from any source must be 50 or more service units.

(e) If you do not have a set of sample data recently collected from your service for the mode and type of service, then you should use one of the other types of sampling plans, such as ready-to-use sampling plans. 


\section{$\S$ 53.03 Must I identify and correct errors with my sample data before I enter them into the template?}

(a) Yes, you must identify and correct errors in your sample data for using the template.

(b) If you are using sample data from previous report years, you should have identified and corrected such errors in developing your estimates then; make sure that you use the final version of your sample data.

(c) Refer to Subsection 67 for identifying and correcting errors in your sampling data during the course of an entire report year as you collect sample data.

(d) If you are using sample data from the current report year, identifying and correcting errors in the sample data now for developing sampling plans saves you from doing it when you develop your estimates later for reporting purposes.

(e) At a minimum, you should conduct the following checks to identifying potential errors:

(1) Overall consistency - UPT and PMT should be closely related across all sample units. For period-based sampling plans, check for errors if cell D26 in the PeriodCalculations worksheet is smaller than 0.5. For interval-based sampling plans, check for errors if cell D20 in the IntervalCalculations worksheet is smaller than 0.5.

(2) Consistency at the level of sampling units - UPT and PMT should both be zero or both be positive. Check for errors if one is zero but the other is positive.

(3) Comparing APTL with route length - Calculate the ratio of PMT over UPT and compare the result to the maximum length of your route. This check is not applicable to demand response services.

(4) If you consider the PPMT option, calculate the ratio of PMT over PPMT for each sample unit; the ratio should not be greater than 1 .

(f) It is in your interest to keep your sample data free of errors. Experience indicates that sample data with errors tend to result in larger sample sizes than sample data free of errors.

\section{$\S$ 53.05 Do interval-based sampling plans have any special requirement for the sample data used in developing them?}

(a) Yes, the sample data must have 2 or more service units for every sample day.

(b) If you sampled 3 one-way bus trips every $2^{\text {nd }}$ day with an annual total sample of 549 trips during your last sampling year, your sample data would meet this requirement. If you have identified and corrected errors in your sample data, you may use them for developing interval-based sampling plans with the template.

\section{\$ 53.07 What other data do I need to use this template?}

(a) If you want to consider the grouping option, you must have data:

(1) on the size of each service group in the number of service units operated during the period for which the sample data were collected, and

(2) on the expected size of each service group for future sampling years.

(3) Follow the instructions in the template on how you should enter the information on group size.

(b) If you want to consider combining the grouping option with the APTL option, you must enter into the template whether you will have reliable 100\% counts of UPT for each service group for estimating your annual PMT. Follow the instructions in the template on where you should enter this information. Your input affects several things: 
(1) The efficiency of your sampling plan. In general your sampling plan is more efficient if you will have reliable $100 \%$ counts of UPT by service group.

(2) How you should estimate your annual PMT. For example, you will need to estimate your annual PMT separately for each service group if you have 100\% counts of UPT by service group. Refer to Subsection 83 for more guidance on estimation.

(3) The information you need to get from the template for determining whether you need to revise your template sampling plan. Refer to Subsection 56 for more guidance on revision of sampling plans.

(c) If you want to consider interval-based sampling plans, you must have information on the annual number of days of service and the daily number of trips.

\section{$\S$ 53.09 What sample data should I use to develop template sampling plans for next year?}

(a) If you are not sampling this year, you should use the sample data from your most recent previous sampling year.

(b) If you are sampling this year, you should use the sample data from this year. It is critical that you process your sample data as they become available. The objective is that you can apply the companion template of this Manual to the cumulative sample data any time during this year. Refer to Section 60 for processing your sample data.

(1) If you are familiar with the companion template of this Manual, you should wait until a couple of weeks before the end of this year to use the template. It is fine if you miss a few service units in your annual NTD sample for this purpose. Note that you will still need to use the full sample to estimate your annual service-consumed data for NTD reporting.

(2) If you are not familiar with the companion template, you should start learning to use the companion template with your sample data from this year. Once you become familiar with the template, you should still wait until a couple of weeks before the end of this year to use the template.

\section{$\$$ 53.11 How does this template deal with fluctuations in sample data from one year to another for a given service?}

(a) The measured statistical variation in APTL or any relevant characteristic of your service fluctuates from one year to another.

(b) Such fluctuations can occur from minor changes in your service or changes in how people use your service even if you do not adjust your service.

(c) Fluctuations can also occur due to errors in data collection as a result of sampling.

(d) This template has built in a $25 \%$ margin of safety against such fluctuations.

(e) This margin of safety is equivalent to increasing the minimum precision level from $10 \%$ to $8.94 \%$ at the same $95 \%$ confidence level.

\section{\$ 53.13 Why do such annual fluctuations in sample data require a margin of safety?}

(a) FTA's $95 \%$ confidence and $10 \%$ precision levels are imposed on your estimates of annual service-consumed data.

(b) The template, on the other hand, can only impose these requirements on your template sampling plans, but not on the estimates you develop with data collected according to your template sampling plans. 
(c) Whether the estimates of annual service-consumed data from the sample data collected according to the template sampling plans meet FTA's requirements depends on how the sample data fluctuate over time:

(1) If the statistical variation is greater in the sample data used to develop your template sampling plan, your estimates will meet FTA's requirements.

(2) If the statistical variation is smaller in the sample data used to develop your template sampling plan, your estimates may not meet FTA's requirements. The margin of safety is built in to prevent such violations from happening.

\section{$\S 53.15$ How does this template ensure adequate sample size for developing template sampling plans in the future?}

(a) Statistical theory shows that a minimum sample size of 50 is required to get an adequately precise estimate of how much APTL or any other relevant characteristic of your service varies across your service.

(b) This template has built in a minimum sample size of 50 for all new template sampling plans.

\section{Subsection 54 - Usage and Output}

\section{§54.01 How do I use this template?}

(a) You must choose Enable Macros upon opening the template.

(b) You must check a checkbox in the Cover Worksheet to develop interval-based sampling plans. The default is for period-based sampling plans.

(c) You must follow the instructions provided in the template.

(d) This template is illustrated with actual sample data in "The NTD Sampling Template with Sample Data.xlsm."

(e) This illustrated template is available for download at the NTD webpage: www.ntdprogram.gov.

\section{$\$ \mathbf{5 4 . 0 3}$ What does the template output include?}

(a) The output includes a set of several sampling plans as a result of the input data you have entered.

(b) The sampling plans in this set differ in their efficiency options and sampling frequency.

(c) For period-based sampling plans, the number of sampling plans in this set varies with the efficiency options that your input data allow. It ranges from 6 sampling plans if you do not consider the PPMT and grouping options to 18 sampling plans if you consider them.

(d) For interval-based sampling plans, the number of sampling plans in this set varies with your weekly service patterns. It ranges from 8 sampling plans if you operate your service 5 days a week to 12 sampling plans if you operate 7 days a week.

(e) The set of sampling plans changes when you change your input data. 


\section{Subsection 55 - Selecting a Sampling Plan}

\section{$\S 55.01 \quad$ What should I consider in selecting one from the set of template sampling}

plans?

(a) When your data allow, you should take the full advantage of the template by considering all possibilities, particularly both period-based and interval-based sampling plans and alternative ways of grouping your service. This exploratory use of the template is especially useful when you have not used template sampling plans before.

(b) Once you have done the exploratory work, you should consider all five elements of a sampling plan:

(1) unit of sampling and measurement,

(2) sampling structure,

(3) efficiency options,

(4) sampling frequency, and

(5) annual sample size.

(c) You should consider tradeoffs among the following characteristics of sampling plans:

(1) sampling cost - it is closely related to the annual sample size and to the unit of sampling and measurement.

(2) complexity of sampling plans - the grouping and PPMT options, for example, make sampling and estimation more complex.

(3) administrative convenience - how a sampling plan matches your institutional arrangements for sampling and data collection purposes.

(d) If you want to choose an interval-based sampling plan for the current report year and want to consider interval-based sampling for future years:

(1) you should consider those plans that require sampling 2 or more service units per sample day. You must have sample data for 2 or more service units for each sample day in order to use the template for developing new interval-based sampling plans in the future. Or

(2) you may increase the daily sample size for a desired sampling frequency (e.g., every $3^{\text {rd }}$ day) if the template results in a daily sample size of 1 for that frequency.

\section{$\S 55.03$ Should I keep a copy of the used template that contains my sample data and my chosen template sampling plan?}

(a) Yes, you should keep a copy of the used template that contains your sample data and the final sampling plans, including the sampling plan you have chosen. It is useful later for several purposes.

(b) You may need it for NTD requirements on record keeping.

(c) You may also need it for the following purposes:

(1) Get information about the sample data for determining whether you need to revise your template sampling plan. Refer to Subsection 56 for guidance on that.

(2) Remind you what you have entered into the template on whether you will have reliable 100\% count of UPT by service group if your template sampling plan is based on the APTL option with service grouping. Refer to Subsection 83 for guidance on estimation under the APTL option. 
\$ 55.05 How does my choice of efficiency options affect my choice of estimation methods?

(a) Your choice of an efficiency option determines your method of estimation.

(b) Follow the guidance on estimation in:

(1) Section 70 if you choose the base option.

(2) Subsection 83 if you choose the APTL option.

(3) Subsection 85 if you choose the PPMT option.

\section{$\S 55.07 \quad$ May I change template sampling plans from one report year to another?}

(a) Suppose that:

(1) you have developed a set of final template sampling plans with sample data from a previous sampling year, and

(2) you are using one of these template sampling plans this year.

(b) The answer depends on whether you are required to revise your current sampling plan for next year. Refer to Subsection 56 on how you may determine the need for revision.

(c) You may change to a different sampling plan from the current set of final template sampling plans if you are not required to revise your current sampling plan.

(d) You may change to a different sampling plan from an entirely new set of template sampling plans if you choose to develop new sampling plans using your sample data from the current report year.

(e) You must not change to a different sampling plan from the current set of template sampling plans if you are required to revise your current sampling plan.

\section{§55.09 May I change sampling plans during a report year?}

(a) You should not change sampling plans during the same report year if you have not made major changes to your service.

(b) You should increase the necessary sample size by $50 \%$ with the same template sampling plan you used during the first part of the year if you have made major changes to your service. For example, if you were sampling 4 one-way bus trips each week before the major changes, you should change to sample 8 one-way bus trips each week after those changes.

(c) Table 55.01 shows this guidance in a tabular format.

Table 55.01. Tabular Guidance on Changing Sampling Plans in a Report year

\begin{tabular}{|l|l|l|}
\hline If you & then you should & by \\
\hline $\begin{array}{l}\text { have not made major } \\
\text { changes to your service }\end{array}$ & $\begin{array}{l}\text { not change your sampling } \\
\text { plan during a report year }\end{array}$ & \\
\hline $\begin{array}{l}\text { you have made major } \\
\text { changes to your service }\end{array}$ & $\begin{array}{l}\text { adjust your sampling plan } \\
\text { during a report year }\end{array}$ & $\begin{array}{l}\text { increasing the necessary } \\
\text { sample size by } 50 \%\end{array}$ \\
\hline
\end{tabular}

\section{§ 55.11 How should I implement a template sampling plan?}

(a) You must not implement a template sampling plan at a unit that is smaller than the unit used in developing the original sampling plan. For example, if your plan is to sample 3 round trips per week, you must not implement it by sampling 3 one-way trips per week. 
(b) You may choose to sample and measure at a unit that is greater than the unit used in developing the original template sampling plan. For example, if your plan is to sample 4 one-way trips per week, you may implement it by sampling 4 round trips per week instead.

(c) You may only want to do (b) during the first year of implementing a template sampling plan. For example, once you have the sample data in round trips from the first year, you should use the sample data from the first year to develop a new template sampling plan in round trips for future years.

\section{Subsection 56 - Revision}

\section{§ 56.01 When does this subsection apply to me?}

(a) When you have used a template sampling plan for at least one year, or

(b) When you have been using a template sampling plan for the first time this year.

\section{$\$ 56.03 \quad$ Why do I need to consider revision?}

(a) Your service and the traveling habits of your customers change naturally over time.

(b) To meet certification requirements:

(1) Such service and habit changes may increase the statistical variation in key quantities (e.g., PMT, APTL) and hence require larger sample sizes.

(2) The sampling plans produced by this template are only certified to meet FTA's requirements of $95 \%$ confidence with $10 \%$ precision for certain sampling cycles, presuming no major changes in your service.

(3) After this time, or after major changes in your service, a new template sampling plan must be developed to maintain certification of compliance with the FTA requirements.

(c) To get more efficient sampling plans:

(1) Such service and habit changes sometimes may reduce the statistical variation of key quantities (e.g., PMT, APTL).

(2) You would be able to take advantage of such reduced variation by revising your template sampling plans using your most recent sample data that reflect these service and habit changes.

\section{§ 56.05 Under what conditions should I revise my template sampling plan?}

(a) If your next sampling year is your mandatory revising year, you must revise your template sampling plans. Or

(b) If you have made major changes to your service since you started using the current template sampling plan, you must consider revising your template sampling plan.

\section{\$ 56.07 How do I know if next year is a mandatory revising year for me?}

(a) If you are required to sample every year, every $6^{\text {th }}$ year is your mandatory revising year. For example, if you used your 2010 NTD sample to develop a template sampling plan, report year 2016 is your mandatory revising year.

(b) If you are required to sample every $3^{\text {rd }}$ year, every $9^{\text {th }}$ year is your mandatory revising year. For example, if you used your 2010 NTD sample to develop a template sampling plan, report year 2019 is your mandatory revising year. 
(c) Follow the Reporting Manual to determine whether you are required to sample every year.

\section{\$ 56.09 What are considered major changes to my service?}

(a) A major change is any change you make to your service that is likely to lead to major changes in the statistical variation in a relevant quantity of your service.

(b) The relevant quantity of your service varies with the sampling options you choose. It is PMT if you choose the base option, APTL if you choose the APTL option, etc.

(c) The following are examples of a major change:

(1) Making transfers fare-free for all passengers.

(2) Adding or cutting express routes to the base of all local service.

(3) Expanding or contracting your service by at least $25 \%$ in vehicle revenue miles.

(4) Service restructuring that affects at least $25 \%$ of your service in vehicle revenue miles.

\section{$\S 56.11$ How do I determine if I must revise my template sampling plan after I have made major changes to my service?}

(a) Enter your sample from the current report year into the companion template as if you were going to develop a new template sampling plan.

(1) Enter your PPMT data if your current template sampling plan follows the PPMT option.

(2) Enter the corresponding grouping data if your current template sampling plan follows the grouping option.

(b) For period-based sampling, go to the PeriodPrecision Worksheet. The index number you need depends on the efficiency option you have chosen for the current template sampling plan. For example, you must use the value in cell D6 of Part A, Figure $56.01(=32.7)$ if your template sampling plan is based on the APTL option with service grouping and if you have chosen to use your $100 \%$ UPT count by group in developing your annual estimates.

(c) For interval-based sampling, go to the IntervalPrecision Worksheet. The index number you need also depends on the efficiency option you have chosen for your current template sampling plan. For example, you must use the value in cell C5 of Part B, Figure 56.01 $(=31.3)$ if your template sampling plan is based on the APTL option.

(d) You must revise your sampling plan if the precision index is smaller than 10.0. 


\begin{tabular}{|c|c|c|c|c|}
\hline & $\mathrm{A}$ & $\mathrm{C}$ & D & $\mathrm{E}$ \\
\hline 1 & \multirow{2}{*}{\multicolumn{4}{|c|}{ Precision Index for Period-Based Sampling }} \\
\hline 2 & & & & \\
\hline 3 & Efficiency Options & No Grouping & Grouping & \\
\hline 4 & Base Option & 14.1 & 15.3 & \\
\hline 5 & APTL Option - 100\% UPT Total & 31.5 & 32.5 & \\
\hline 6 & APTL Option - 100\% UPT by Group & $\mathrm{N} / \mathrm{A}$ & 32.7 & \\
\hline 7 & PPMT Option & 24.3 & 30.9 & \\
\hline 8 & \multirow{3}{*}{\multicolumn{4}{|c|}{$\begin{array}{l}\text { Notes: } \\
\text { 1. APTL Option - } 100 \% \text { UPT Total applies if cell M52 = } 0 \text { in the Periodlnput Worksheet. } \\
\text { 2. APTL Option - } 100 \% \text { UPT by Group applies otherwise. }\end{array}$}} \\
\hline 9 & & & & \\
\hline 10 & & & & \\
\hline
\end{tabular}

A. PeriodPrecision Worksheet

\begin{tabular}{|c|c|c|c|c|}
\hline & A & C & $\mathrm{D}$ & $\mathrm{E}$ \\
\hline 1 & \multicolumn{4}{|c|}{ Precision Index for Interval-Based Sampling } \\
\hline 2 & & & & \\
\hline 3 & Efficiency Options & No Grouping & & \\
\hline 4 & Base Option & 14.0 & & \\
\hline 5 & APTL Option - 100\% UPT Total & 31.3 & & \\
\hline
\end{tabular}

Figure 56.01. Layout of the PeriodPrecision and IntervalPrecision Worksheets

\section{Subsection 57 - Certification}

\section{§ 57.01 How do I certify my template sampling plan?}

(a) You must certify that that your template sampling plan meets FTA's 95\% confidence and $10 \%$ precision levels through your annual reporting process.

(b) You should follow the current Reporting Manual on how exactly you may do that.

(c) This certification of your template sampling plan through the annual reporting process is conditional:

(1) Your template sampling plan must use the $25 \%$ margin of safety according to Subsection 53.

(2) You revise your template sampling plans over time according to Subsection 56. 


\section{SECTION 60 - DATA COLLECTION}

\section{Subsection 61 - General Directions}

\section{\$ 61.01 What are the basic elements of collecting sample data?}

(a) Selecting a sample at random according to your sampling plan (Subsection 63).

(b) Collecting data from the random sample (Subsection 65).

(c) Identifying and correcting any errors in the sample data (Subsection 67).

$\S 61.03$ What criteria does this section cover to ensure that estimates of annual serviceconsumed data meet FTA's $95 \%$ confidence and $10 \%$ precision levels?

(a) Your sampling process covers your entire service.

(b) You select your sample at random according to your chosen ready-to-use sampling plan or your template sampling plan.

(c) Your data-collection process is designed to avoid errors from happening.

(d) Your data-collection is designed to identify and correct errors when they occur.

\section{Subsection 63 - Selecting a Sample at Random}

§ 63.01 What are the basic elements of selecting a sample at random?

(a) Choosing a method with which you select a sample at random.

(b) Developing lists of service units that you expect to operate:

(1) A single list of all services if your sampling plan is not based on service grouping.

(2) One list for each service group if your sampling plan is based on service grouping.

(c) Selecting a sample at random from each list with the chosen method.

\section{§ 63.03 What method may I use for random sampling?}

(a) You may use the traditional method based on a table of random numbers.

(b) You may use any other method for random sampling as long as it meets these two criteria:

(1) sampling under the method is random.

(2) sampling under the method is without replacement. Without replacement means that the method will not select the same service unit more than once.

§ 63.05 What is a table of random numbers?

(a) It is a list of integers whose frequency and sequence of appearance in the list have been determined entirely by chance.

(b) For convenience and simplicity in use, published tables of random numbers usually appear in the form of separate columns of five-digit numbers. Both rows and columns may be consecutively numbered for easy reference. Table 63.01 shows an example.

(c) Appendix 98 is a comprehensive table of random numbers you may use for random sampling if you choose to use this method. 
Table 63.01. Example of a Table of Random Numbers

\begin{tabular}{|c|c|c|c|c|c|}
\hline Rows & $(1)$ & $(2)$ & $(3)$ & $(4)$ & $(5)$ \\
\hline 1 & 10480 & 15011 & $01 \$ 536$ & 02011 & 81647 \\
\hline 2 & 22368 & 46573 & 25595 & 85393 & 30995 \\
\hline 3 & 24130 & 48360 & 22527 & 97265 & 76393 \\
\hline 4 & 42167 & 93093 & 06243 & 61680 & 07856 \\
\hline 5 & 37570 & 39975 & 81837 & 16656 & 06121 \\
\hline
\end{tabular}

\section{§ 63.07 What is the list of all service units that I expect to operate?}

(a) It is the amount of revenue service that you expect to operate.

(b) It is measured with the following characteristics:

(1) It is in the unit of sampling and measurement of the sampling plan you have chosen.

(2) It is for the duration corresponding to the sampling frequency that you have chosen for your sampling plan. The duration would be a day for interval-based sampling but a week, a month, or a quarter for period-based sampling.

(3) It is for each of the service groups you have defined if your sampling plan is based on service grouping.

(c) For scheduled services, the list must include:

(1) all service units that are listed on the schedule, and

(2) all service units that are not on the schedule but are expected to be operated, such as trippers, shuttles, and other special operations.

(d) It would be the number of one-way bus trips you expect to operate in a week by your MB express routes, for example, if:

(1) your sampling plan is in terms of one-way bus trips,

(2) you have chosen weekly sampling for your MB service,

(3) your sampling plan involves service grouping, and

(4) you use express routes as one of the groups.

\section{§63.09 How do I develop the list of all service units for using a table of random numbers?}

(a) Suppose that:

(1) you have chosen weekly sampling for your vanpool service,

(2) your sampling plan requires 2 vanpool-days per week,

(3) you want to select a random sample for next week, and

(4) you have 101 vanpools and each of them is expected to operate every day next week.

(b) One way to develop the list would be to assign a serial number of four-digits to each vanpool-day for all 707 combinations of vanpools and service days. The first digit would represent the day of week with 1 for Monday and 7 for Sunday. The other three digits would represent vanpool numbers ranging from 001 through 101.

(c) The vanpool numbers may differ from what you use for your internal purposes. Make sure that you have a one-to-one list between the internal vanpool numbers and the new serial numbers if they differ.

(d) The serial numbers on a given day must be consecutive without any gaps. 
(e) Write down just the first and the last for each day of week so that you will know the range of numbers. For example, the range for Monday is 1001-1101.

(f) You should summarize these serial numbers in a summary table as in Table 63.03.

Table 63.03. Example of a Summary Table of Serial Numbers

\begin{tabular}{cc}
\hline Day of Week & Range of Serial Numbers \\
\hline 1 (Monday) & $1001-1101$ \\
2 & $2001-2101$ \\
3 & $3001-3101$ \\
4 & $4001-4101$ \\
5 & $5001-5101$ \\
6 & $6001-6101$ \\
7 & $7001-7101$ \\
\hline
\end{tabular}

\section{$\S 63.11 \quad$ How do I use a table of random numbers?}

(a) Suppose that:

(1) you have 101 vanpools that you expect to operate every day of next week,

(2) you have numbered the vanpool days for all vanpools as shown in Table 63.03, and

(3) your sampling plan for your vanpool service requires 2 vanpool days per week.

(b) You must work with a constant length of digits from a table of random numbers for sampling. This constant length is given by the number of digits in the longest serial number you have assigned to members of the list of all service units.

(1) This constant length would be 4 for the example in Table 63.03 because all serial numbers are four-digits long.

(2) This constant length would be 5 if your serial numbers vary in length, ranging from one to five digits.

(c) You must combine adjacent digits from the table of random numbers as needed to obtain a two-, three-, or four-digit number, or any other length number from the table.

(1) You may choose to work with rows or columns in combining adjacent digits. You may start with any row if you choose to work with rows. You may start with any column if you choose to work with columns. You may also choose to work from right to left or from left to right if you choose to work with rows.

(2) If you choose to work with rows and to start with row 1, for example, the first 9 fourdigit numbers from Table 63.01 would be:

1048, 0480, 4801, 8015, 0150, 1501, 5011, 0110, and 1101.

(d) You must continue forming four-digit numbers until you find two four-digit numbers from the table of random numbers that are in the summary table of serial numbers (Table 63.03). In the above example, these two numbers are 1048 and 1101. That is, you should select vanpools 48 and 101 and collect sample data from them on Monday of next week.

(e) Once you are done sampling for a week, you should mark where you stopped forming four-digit numbers in the table of random numbers. For example, the symbol, $\$$, has been 
inserted between digits 1 and 5 in row 1 and column 3 of Table 63.01. You should start the above process for a later week after that mark.

(f) Once you are done sampling for a year, you should mark where you stopped forming four-digit numbers in the table of random numbers if you plan to use this method of random sampling in the future.

(1) If you number your service units exactly as for a previous sampling year, you should start the above process for a new sampling year after the last mark of your previous sampling year.

(2) If you number your service units with a different approach for a new sampling year, you may start the above process anywhere in the table of random numbers.

\$ 63.13 What are the pros and cons of using a table of random numbers?

(a) Using a table of random numbers for random sampling has the following advantages:

(1) It is applicable to all situations.

(2) You do not need to list explicitly all service units.

(b) It has the following disadvantages:

(1) You must number your service units consecutively without gaps, at least within each subset of your service units. Subsets are formed by route, type of service days, etc.

(2) You may not be able to take advantage of the serial numbers you have already assigned to your service units for internal purposes.

(3) It can be difficult to use if the serial numbers have a large number of digits.

\section{\$ 63.15 What information should I keep from my sampling process?}

(a) You should have an auditable record of your sampling process. That record should cover the following:

(1) A written description of the sampling plan.

(2) A written procedure for your method of selecting a sample at random.

(3) The list of all service units from which you selected a sample at random for each act of sampling. For example, you should have 52 such lists if you did weekly sampling for an entire year.

(4) The random sample.

\section{Subsection 65 - Collecting Data from the Random Sample}

\section{§ 65.01 What method may I use to collect the sample data?}

(a) One common method involves one or more ride checkers observing and recording passenger activities while riding in a transit vehicle.

(b) An increasingly common method uses APCs to record passenger activities instead.

(c) You may use one of these or any other method to collect the data from each service unit of your random sample as long as your method meets these criteria:

(1) it obtains data from direct measurement or direct observation without passenger intercept,

(2) it provides data you must have to determine PMT for each service unit, and

(3) it meets the requirements on measurement accuracy in the NTD Reporting Manual. 
(a) You may use the load-based approach. It determines PMT by multiplying the number of passengers onboard a transit vehicle between each pair of consecutive stops by the distance between these stops.

(b) You may also use the distance-based approach. It determines PMT by keeping track of the distance traveled by each passenger carried by a service unit of your random sample.

\section{$\S 65.05$ What data items must I collect to use the load-based approach for scheduled services?}

(a) You must collect the required data items separately for each one-way vehicle trip in your service unit.

(1) If your service unit is a round-trip bus trip, for example, it has two one-way vehicle trips.

(2) If a service unit is a one-way train trip with three passenger cars, for example, it has three one-way vehicle trips.

(b) The following data items are required for each one-way vehicle trip:

(1) the number of people who boarded at each stop,

(2) the number of people who alighted at each stop,

(3) the distance between any pair of consecutive stops at which boardings or alightings occurred,

(4) the number of people onboard the vehicle between any pair of consecutive stops,

(5) the number of people who stayed on from the previous one-way vehicle trip, and

(6) the number of people who remained on the vehicle at the last stop.

\section{$\S$ 65.07 What data must I collect to use the load-based approach for non-scheduled} services?

(a) For commuter vanpool, the following data items are required for each direction of commuting:

(1) the number of people who boarded at each pick-up location,

(2) the number of people who alighted at each drop-off location, and

(3) the distance between any pair of consecutive stops at which pick-up or drop-off occurred.

(b) For demand response (DR and DT), you must collect the required data items continuously during the entire vehicle day sampled:

(1) the odometer reading at each pick-up location,

(2) the number of people onboard between each pair of consecutive locations at which pick-up or drop-off occurred, and

(3) the odometer reading at each drop-off location.

(c) You should treat non-commuter vanpool as demand response for data collection.

§ 65.09 How should I determine between-stop distances for the load-based approach?

(a) You should avoid using maps to estimate between-stop distances under all circumstances.

(b) For services without designated stops, including demand response (DR and DT), jitney (JT), vanpool (VP), or fixed-route services without designated stops: 
(1) If available, you should always use the onboard odometer to determine the betweenstop distances for each service unit in your random sample. You should make sure that the vehicles for the sampled service unit have a working odometer onboard before they start for the sampled service unit.

(2) If your vehicle does not have an onboard odometer, you should determine the distance by retracing the path and the stops by automobile.

(3) You should record your odometer readings at least to one-tenth of a mile.

(c) For ferryboat (FB), aerial tramway (TR) and all rail services, you may want to predetermine the between-station distances for all routes and directions.

(d) For fixed-route services with designated stops, you should use one of two approaches:

(1) predetermine the between-stop distances for all routes and directions, or

(2) record the onboard odometer readings at individual stops.

(e) You may use different methods to predetermine the between-stop distances:

(1) use an up-to-date GIS of your network of routes and stops, or

(2) record the odometer readings while you drive through all of your routes and related deviations and directions.

(f) If you predetermine between-stop distances, you must keep them updated to reflect any changes in your services.

\$ 65.11 What additional data should I collect to identify each service unit of my sample?

(a) You must record the date and the type of service days for all cases.

(b) For commuter rail (CR), heavy rail (HR), and light rail (LR), you must also record the weekday time period that you will be reporting to the NTD for weekdays.

(c) If your sampling plan is based on the PPMT option, you must record route identification.

(d) If your sampling plan is based on service grouping, you should also record information that is necessary to determine group membership of each service unit in the random sample.

\section{§ 65.13 When do I use the different approaches to determining PMT?}

(a) The load-based approach is applicable to all circumstances. Under conditions of heavy loads or high boarding volumes, however, extra care must be taken to maintain the required level of measurement accuracy.

(b) The distance-based approach is useful under several circumstances. For examples:

(1) All passengers board and alight at the same locations, such as inclined plane (IP), aerial tramway (TR), and most ferryboat (FB) operations.

(2) Services with a small number of passengers who board and alight at a small number of stops, such as demand response (DR and DT).

(3) Services with a small number of frequent passengers who board and alight at a small number of stops, such as commuter vanpool.

(4) Any service with a ticketing system that keeps track of the origin and destination for every boarding with a known distance.

§ 65.15 What instrument should I use to collect the data for the distance-based approach?

(a) You may use any instrument that you have designed as long as you can use it to record the required data items correctly. 
(b) If you use human ride checkers, the instrument may be a piece of paper and a pencil or it may be a hand-held device.

(c) Appendix 92 provides an example of both blank and filled-out paper instruments for the distance-based approach.

\section{\$ 65.17 What instrument should I use to collect data for the load-based approach?}

(a) You may use any instrument that you have designed as long as you can record the required data items correctly.

(b) If you use APCs, the instrument would be computer software and hardware that records the counts and other data items transmitted from the APCs.

(c) If you use human ride checkers, the instrument may be a piece of paper and a pencil or it may be a hand-held device.

(d) If you use a hand-held device, it is critical that the unit accepts counts of boardings and alightings that may not be equal for a one-way vehicle trip.

(e) The instrument you design may take slightly different formats for different services. Three examples of blank and filled-out instruments are shown as appendices:

(1) demand response - Appendix 93.

(2) commuter vanpool - Appendix 94.

(3) fixed-route services - Appendix 95.

(f) If you are going to use one of these example instruments, you should study it carefully before reading the following guidance.

\section{$\S 65.19$ What pre-survey procedures should I follow if I use a paper instrument and human ride checkers?}

(a) You should use an appropriate survey sheet for each service unit selected in the sample. This survey sheet may be one of those from the appendices or one that you have designed, but it is important that you select a survey sheet that is designed for the service to be surveyed.

(b) For non-scheduled services, including demand response (DR and DT) and vanpool (VP), a survey sheet should be used for each driver of the survey vehicle during an entire day.

(c) For scheduled services, a survey sheet should be used for each one-way vehicle trip of your service unit that you have chosen for your sampling plan.

(d) The ride checker(s) should carry several extra survey sheets in the event that extra sheets are needed.

(e) You should learn about the likely load and boarding volumes for each service unit to be surveyed.

(1) You may need to use more than one ride checker under conditions of high loads and high boarding volumes.

(2) If more than one ride checker is used, the separate survey sheets should also be labeled with the door(s) that each ride checker is responsible for.

(f) If you rely on the onboard odometer to determine the between-stop distances, make sure that it works properly. If you cannot fix a malfunctioning odometer in time for a ride check, use a different vehicle with a working odometer.

(g) Before going into the field, the survey supervisor should fill in the data items that identify the service unit to be surveyed. If your service unit has more than one one-way vehicle trip, identify each one-way vehicle trip separately. 
(h) If you rely on predetermined between-stop distances, the survey supervisor should also use the survey sheet(s) in Appendix 95 to:

(1) Fill in the stop numbers in column (7) and stop descriptions in column (8) for all stops.

(2) Cross out column (9) to avoid confusion in the field.

(i) You should write the page numbers in the box in the lower right-hand corner of each survey sheet if you use more than one survey sheet for a given one-way vehicle trip.

\section{$\S$ 65.21 What manual survey procedures should I follow for demand response (DR and DT) with the distance-based approach?}

(a) You may use the survey sheet for the distance-based approach in Appendix 92.

(b) You must record individual trips by each pair of origin and destination. The filled-out form in Appendix 92 illustrates three cases of pick-up and drop-off patterns:

(1) The first pick-up illustrates the "one origin-many destinations" case. The driver picked up 16 passengers at $1020 \mathrm{~J}$ Street. He immediately distributed these passengers according to their three drop-off destinations under item (8).

(2) The second pick-up illustrates the "one origin-one destination" case. At the next pick-up address, 506 10th Street, 17 passengers boarded and all were driven to the same destination. Here, a single line records all the necessary information.

(3) The last two pick-ups illustrate a "many origins-one destination" case. Here, the driver records the pick-up addresses and repeats the destinations; that is, he handles each as an individual trip.

(c) You must record the odometer readings for each pick-up and drop-off.

(1) Item (7), "Pick-Up Odometer Reading," should be recorded immediately upon picking up the passenger(s).

(2) Item (8), "Drop-Off Odometer Reading," should be recorded immediately upon dropping off the passengers.

(3) You should also record the odometer readings to at least one-tenth of a mile when the odometer allows.

(d) You should use additional pages if a service unit involves more stops than are given on a page. You should write the page numbers in the box in the lower right-hand corner.

(e) As an alternative to data recording by the driver, the driver can call in the information to the dispatcher if you have two-way radio communications on all vehicles.

(1) When the driver arrives at a pick-up point, he can call in items (5)-(8).

(2) When he arrives at each destination, he can call in item (9) and verify how many passengers were dropped off at that destination.

\section{$\S$ 65.23 What manual survey procedures should I follow for demand response (DR and DT) with the load-based approach?}

(a) You may use the survey sheet for the load-based approach for demand response in Appendix 93.

(b) For each pick-up or drop-off, you must record the following:

(1) whether it is a pick-up or drop-off in column (5),

(2) a description of the location in column (6),

(3) the odometer reading in column (7), and

(4) the leaving load in column (8). 
(c) The filled-out form in Appendix 93 shows the recorded information for the example shown in Appendix 92. The leaving load at the last drop-off location should be 0 .

(d) You should record the odometer readings to at least one-tenth of a mile.

(e) You should use additional pages if a service unit involves more stops than are given on a page. You should write the page numbers in the box in the lower right-hand corner.

(f) As an alternative to data recording by the driver, the driver can call in the information to the dispatcher if you have two-way radio communications on all vehicles. Before leaving each pick-up or drop-off location, the driver can call in items (5)-(8).

\section{$\S$ 65.25 What manual survey procedures should I follow for commuter vanpool with the load-based approach?}

(a) Suppose that you have chosen to sample on a monthly basis.

(b) Before a new month starts, you should communicate with the driver of each sampled vanpool about the days on which he must collect sample data during the new month.

(c) For each sampled vanpool day, the driver should fill in the identification data before he leaves home, including the date, the day of week, and the vanpool number.

(d) The driver is required to record the travel data, including the van odometer reading whenever any rider gets on or off the van along with the number of riders who get on the van and the number of riders who get off the van. This recording is to be done separately for travel to work and travel from work. The filled-out form in Appendix 94 shows the travel data for a vanpool of 6 riders with a round-trip-distance of 78 miles. The driver picks up all 5 riders at a single location but drops them off at three different locations in the morning. In the afternoon, the process reverses itself.

(1) Once the driver gets on the van in the morning, he should enter 1 in the ON column (6) and record the odometer reading at 29,366.0 in column (8).

(2) He then leaves for picking-up 5 fellow vanpoolers at another location. Once they get on the van, he should enter 5 in (6), and record the odometer reading at 369.1 in (8) before departing that location.

(3) At the first drop-off location, 3 vanpoolers get off. Before leaving, the driver should enter 3 in the OFF column (7), and record the odometer reading at 395.3 in (8).

(4) One vanpooler gets of the van at each of the next two drop-off locations. Before leaving these locations, the driver should enter 1 in the OFF column, and record the odometer reading at 396.8 and 397.5, respectively.

(5) Finally, the driver arrives at his own destination. Before he leaves the van, he should record the odometer reading again at 405.0.

(e) The day after each sample day, you should communicate with each driver involved in collecting sample data to determine if he actually recorded the sample data. Sample data may be not recorded for a variety of reasons.

(1) If the data were collected, the driver should send the filled-out survey sheet immediately,

(2) If the driver forgot to collect the data, he should be asked to collect the data next day,

(3) If the driver refused to collect the data or the vanpool has been terminated, a replacement vanpool day should be selected at random. 


\section{§ 65.27 What instructions should I give my ride checkers if I do not use predetermined between-stop distances for fixed-route services?}

(a) Take enough copies of the survey sheet in Appendix 95 or an alternative sheet.

(b) Use separate survey sheets for separate one-way vehicle trips if your service unit has more than one one-way vehicle trip.

(c) Use additional survey sheets if needed for a given one-way trip.

(d) Board the transit vehicle at the beginning point of the service unit and position yourself so that you can observe the doors for which you are responsible.

(e) Before the vehicle leaves the beginning point, record:

(1) Stop \#1 in column (7),

(2) stop description of the beginning point in column (8),

(3) odometer reading to at least one-tenth of a mile in column (9),

(4) number of passengers who have stayed onboard from the last trip (13), and

(5) number of passengers boarded in (10), including the passengers who have stayed onboard from the last trip in (e)(4).

(f) When the vehicle leaves the beginning point, record the number of passengers onboard in column (12), including any passengers who have stayed onboard from the last trip.

(g) Only at points where the vehicle stops during the service unit, record:

(1) stop number in (7),

(2) stop description in (8),

(3) odometer reading (from the driver) in (9),

(4) passengers boarded in (10), and

(5) passengers alighted in (11).

(h) Between stops, count the number of passengers on board (12). You should record this number as the leaving load. For example, between stops 2 and 3 you should record this number in the row for stop 2 rather than in the row for stop 3.

(i) At the end point of each one-way trip, record the number of passengers who will stay on board to the next trip in column (14) and in column (11) as passengers alighted.

\section{§ 65.29 What steps should I take if I fail to collect the sample data from a particular unit?}

(a) You may fail to collect the required sample data from any particular unit in your sample:

(1) The unit may be canceled for a variety of operational reasons.

(2) The ride checker(s) may fail to show up for the unit.

(3) The ride checker(s) may fail to collect the data.

(b) You must replace the missed unit as soon as possible on the same type of service days.

(c) For period-based sampling:

(1) If the rest of the sampling period (a week, a month, or a quarter) has at least one service day of the same type as the missed service unit, select one service unit from the next service day of the same type. For example, if the missed service unit is on the second Monday of a month and your sampling is monthly, you should get a replacement unit from the third Monday of the month.

(2) If the rest of the sampling period does not have any service day of the same type left, select one service unit from the first service day of the same type during the next sampling period. For example, if the missed service unit is on a Monday and your sampling is weekly, you should get a replacement unit from the next Monday.

(d) For interval-based sampling: 
(1) If the rest of the report year has at least one service day of the same type as the missed service unit, select one service unit from the next service day of the same type.

(2) If the rest of the report year does not have any service day of the same type left, select one service unit from the next day of service.

(e) The replacement unit must be selected at random in all cases.

\section{Subsection 67 - Correcting Errors in the Raw Sample Data}

§ 67.01 What steps should I take after I have collected the sample data?

(a) Design a format for recording your sample data.

(b) Enter the raw data.

(c) Process the entered data.

(d) Identify errors in the entered data.

(e) Identify sources of the data errors, if any.

(f) Correct the data errors, if any.

(g) What you should do within each step depends on your situation. To be specific, the following example is used for the rest of this subsection:

(1) you collect sample data with human ride checkers from one-way bus trips with designated stops,

(2) you use the load-based approach to determining PMT,

(3) you use predetermined between-stop distances, and

(4) you have collected sample data from a route whose longest one-way trip is 4 miles.

\section{\$ 67.03 What should I consider in designing the format for data recording?}

(a) The data items you have collected to determine PMT. The exact data items depend on your service and the approach you have taken to determine PMT.

(b) The data items that identify the service units in your sample.

(c) A format that is easy for data analysis.

(d) Suppose that you have designed a format in Table 67.01. With this format, you enter the field data (7)-(14) as they appear on the field survey sheet shown in the filled-out form in Appendix 95. Item (8) is not shown due to space limit. The identification data items at the top of the survey sheet (1)-(6) are repeated for each stop of this one-way trip. 
Table 67.01. Example Format for Data Recording

\begin{tabular}{|c|c|c|c|c|c|c|c|c|c|c|c|c|}
\hline Date & $\begin{array}{l}\text { Day of } \\
\text { Week }\end{array}$ & $\begin{array}{l}\text { Time } \\
\text { Period }\end{array}$ & $\begin{array}{c}\text { Route } \\
\text { No. }\end{array}$ & $\begin{array}{l}\text { Trip } \\
\text { No. } \\
\end{array}$ & Direction & $\begin{array}{c}\text { Stop } \\
\text { Sequence }\end{array}$ & $\begin{array}{l}\text { Distance to } \\
\text { Next Stop }\end{array}$ & $\begin{array}{c}\text { No. of } \\
\text { Pass. } \\
\text { Boarded }\end{array}$ & $\begin{array}{c}\text { No. of } \\
\text { Pass. } \\
\text { Alighted }\end{array}$ & \begin{tabular}{|c|} 
No. of \\
Pass. On \\
Board \\
(Leaving \\
Load) \\
\end{tabular} & \begin{tabular}{|c} 
No. of Pass. \\
from \\
Previous \\
Trip (Stop 1 \\
only) \\
\end{tabular} & $\begin{array}{c}\text { No. of Pass. } \\
\text { Continuing } \\
\text { to Next Trip } \\
\text { (Last Stop } \\
\text { only) }\end{array}$ \\
\hline$(1)$ & (2) & (3) & (4) & $(5)$ & (6) & (7) & (9) & (10) & (11) & (12) & (13) & (14) \\
\hline $10 / 13 / 05$ & Thur & Midday & 11 & 408 & Outbound & 1 & 0.3 & 20 & 0 & 20 & 2 & \\
\hline $10 / 13 / 05$ & Thur & Midday & 11 & 408 & Outbound & 2 & 0.7 & 2 & 1 & 21 & & \\
\hline $10 / 13 / 05$ & Thur & Midday & 11 & 408 & Outbound & 3 & 0.6 & 0 & 2 & 19 & & \\
\hline $10 / 13 / 05$ & Thur & Midday & 11 & 408 & Outbound & 4 & 0.3 & 1 & 3 & 17 & & \\
\hline $10 / 13 / 05$ & Thur & Midday & 11 & 408 & Outbound & 5 & 0.5 & 1 & 10 & 8 & & \\
\hline $10 / 13 / 05$ & Thur & Midday & 11 & 408 & Outbound & 6 & 0.8 & 0 & 2 & 6 & & \\
\hline $10 / 13 / 05$ & Thur & Midday & 11 & 408 & Outbound & 7 & 0.2 & 0 & 1 & 5 & & \\
\hline $10 / 13 / 05$ & Thur & Midday & 11 & 408 & Outbound & 8 & 0.1 & 0 & 2 & 3 & & \\
\hline $10 / 13 / 05$ & Thur & Midday & 11 & 408 & Outbound & 9 & 0.1 & 0 & 2 & 1 & & \\
\hline $10 / 13 / 05$ & Thur & Midday & 11 & 408 & Outbound & 10 & 0.3 & 0 & 0 & 1 & & \\
\hline $10 / 13 / 05$ & Thur & Midday & 11 & 408 & Outbound & 11 & 0.1 & 0 & 0 & 1 & & \\
\hline $10 / 13 / 05$ & Thur & Midday & 11 & 408 & Outbound & 12 & 0.0 & 0 & 1 & 1 & & 1 \\
\hline
\end{tabular}

\section{§ 67.05 When should I enter my sample data?}

(a) You should have the sample data from each service unit entered immediately after your ride checker(s) has come back from the field.

(b) This has a number of advantages:

(1) reducing the chance of loss of information,

(2) increasing the chance of correcting data errors, if any,

(3) being ready to use the cumulative sample data any time during the sampling year, and

(4) minimizing the rush at the end of the year.

\section{§ 67.07 How should I process the entered data?}

(a) You should process the data as follows immediately after you have entered them. If your service unit has more than one one-way vehicle trip, you should process the data for individual one-way vehicle trips:

(1) Sum the number of passengers boarded at individual stops in column (10) of Table 67.01 to get the total number of passengers boarded (UPT). It is 24 in this case.

(2) Sum the number of passengers alighted at individual stops in column (11) of Table 67.01 to get the total number of passengers alighted. It is 24 in this case.

(3) Calculate the load between every pair of two consecutive stops. Table 67.03 shows an example and the formulas for calculating leaving loads and arriving loads.

(4) Calculate PMT for each pair of consecutive stops by multiplying the calculated load with the between-stop distance. Table 67.03 also shows the calculation of PMT with both leaving loads and arriving loads.

(5) Calculate total PMT. It is 47.8 miles in this case.

(6) Divide total PMT by the total number of passengers boarded to get APTL. It is 1.99 miles in this case.

(7) Calculate vehicle trip length by summing up the predetermined between-stop distances in column (9). It is 4.0 miles in this case.

(b) If your sampling plan is based on the PPMT option, you should also process the data as follows immediately to calculate the ratio of PMT to PPMT for each service unit: 
(1) Calculate the average route length for each route.

(2) Calculate PPMT by multiplying UPT by average route length.

(3) Divide PMT by PPMT to get the ratio for each service unit.

(4) Follow Subsection 85 on calculating average route length and PPMT.

\section{Table 67.03. Calculation of Loads and PMT}

\begin{tabular}{|c|c|c|c|c|c|c|c|}
\hline $\begin{array}{l}\text { Load } \\
\text { Type }\end{array}$ & $\begin{array}{l}\text { Stop } \\
\text { Sequence }\end{array}$ & $\begin{array}{l}\text { Distance to } \\
\text { Next Stop }\end{array}$ & $\begin{array}{c}\text { No. of } \\
\text { Pass. } \\
\text { Boarded }\end{array}$ & $\begin{array}{l}\text { No. of Pass. } \\
\text { Alighted }\end{array}$ & $\begin{array}{c}\text { No. of Pass. } \\
\text { from Previous } \\
\text { Trip } \\
\end{array}$ & $\begin{array}{l}\text { Calculated } \\
\text { Load }\end{array}$ & PMT \\
\hline \multirow{14}{*}{ 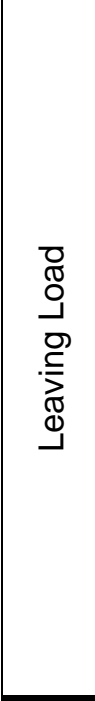 } & 1 & 0.3 & 20 & 0 & 2 & 20 & 6.0 \\
\hline & 2 & 0.7 & 2 & 1 & & 21 & 14.7 \\
\hline & 3 & 0.6 & 0 & 2 & & 19 & 11.4 \\
\hline & 4 & 0.3 & 1 & 3 & & 17 & 5.1 \\
\hline & 5 & 0.5 & 1 & 10 & & 8 & 4.0 \\
\hline & 6 & 0.8 & 0 & 2 & & 6 & 4.8 \\
\hline & 7 & 0.2 & 0 & 1 & & 5 & 1.0 \\
\hline & 8 & 0.1 & 0 & 2 & & 3 & 0.3 \\
\hline & 9 & 0.1 & 0 & 2 & & 1 & 0.1 \\
\hline & 10 & 0.3 & 0 & 0 & & 1 & 0.3 \\
\hline & 11 & 0.1 & 0 & 0 & & 1 & 0.1 \\
\hline & 12 & 0.0 & 0 & 1 & & 0 & 0.0 \\
\hline & Total & 4.0 & 24 & 24 & 2 & $\mathrm{~N} / \mathrm{A}$ & 47.8 \\
\hline & \multicolumn{2}{|c|}{$\begin{array}{l}\text { Load for Stop } 1= \\
\text { Load for Other Stops }=\end{array}$} & \multicolumn{5}{|c|}{$\begin{array}{l}\text { Current Boarding } \\
\text { Previous Load }+ \text { Current Boarding - Current Alighting }\end{array}$} \\
\hline \multirow{15}{*}{ 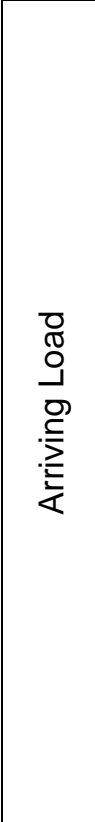 } & $\begin{array}{l}\text { Stop } \\
\text { Sequence }\end{array}$ & $\begin{array}{l}\text { Distance from } \\
\text { Previous Stop }\end{array}$ & $\begin{array}{c}\text { No. of } \\
\text { Pass. } \\
\text { Boarded }\end{array}$ & $\begin{array}{l}\text { No. of Pass. } \\
\text { Alighted }\end{array}$ & $\begin{array}{c}\text { No. of Pass. } \\
\text { from Previous } \\
\text { Trip }\end{array}$ & $\begin{array}{l}\text { Calculated } \\
\text { Load }\end{array}$ & PMT \\
\hline & 1 & 0.0 & 20 & 0 & 2 & 0 & 0.0 \\
\hline & 2 & 0.3 & 2 & 1 & & 20 & 6.0 \\
\hline & 3 & 0.7 & 0 & 2 & & 21 & 14.7 \\
\hline & 4 & 0.6 & 1 & 3 & & 19 & 11.4 \\
\hline & 5 & 0.3 & 1 & 10 & & 17 & 5.1 \\
\hline & 6 & 0.5 & 0 & 2 & & 8 & 4.0 \\
\hline & 7 & 0.8 & 0 & 1 & & 6 & 4.8 \\
\hline & 8 & 0.2 & 0 & 2 & & 5 & 1.0 \\
\hline & 9 & 0.1 & 0 & 2 & & 3 & 0.3 \\
\hline & 10 & 0.1 & 0 & 0 & & 1 & 0.1 \\
\hline & 11 & 0.3 & 0 & 0 & & 1 & 0.3 \\
\hline & 12 & 0.1 & 0 & 1 & & 1 & 0.1 \\
\hline & Total & 4.0 & 24 & 24 & 2 & $\mathrm{~N} / \mathrm{A}$ & 47.8 \\
\hline & \multicolumn{2}{|c|}{$\begin{array}{l}\text { Load for Stop } 1= \\
\text { Load for Other Stops = }\end{array}$} & \multicolumn{5}{|l|}{$\begin{array}{l}0 \\
\text { Prev }\end{array}$} \\
\hline
\end{tabular}


(a) Make sure that UPT and PMT are consistent for each service unit in the sample. For example, they should both be zero or both be positive.

(b) Compare vehicle trip length with the longest actual length of the route. Vehicle trip length must not exceed the longest route length.

(c) Compare APTL with vehicle trip length and route length. APTL must not exceed either.

(d) Compare the total number of passengers boarded with the total number of passengers alighted. They must be equal.

(e) Examine the calculated load at the end point of the trip. It must be zero for leaving loads.

(f) If calculated, examine the PMT to PPMT ratio for each service unit. It must not exceed 1.

\section{§ 67.11 How do I identify the sources of any errors?}

(a) If you have identified any error from the previous step, you should start with the calculations you did in processing the sample data to determine if an error is a calculation error or an error in the sample data.

(b) Check if you have correctly calculated loads, UPT, PMT, and APTL.

(c) Check if you have used between-stop distances and loads consistently:

(1) If you use leaving loads, you must use the distance to the next stop. The distance should be zero for the ending point of a one-way trip.

(2) If you use arriving loads, you must use the distance from the previous stop. The distance should be zero for the beginning point of a trip.

(d) Check the predetermined between-stop distances you entered.

(e) Compare your calculated load with the observed load from the field.

\section{§ 67.13 How do I correct any errors?}

(a) Table 67.05 shows an example of sample data for a one-way trip where PMT is based on calculated leaving loads. The first row of Table 67.07 shows the vehicle trip length, UPT, PMT, APTL, and the ratio of PMT/PPMT for this trip.

(b) It has the following errors:

(1) Vehicle trip length $>$ route length.

(2) APTL > route length.

(3) Total boardings in column (10) < total alightings in column (11).

(4) The load at the end stop $<0$.

(c) Error (b)(1) clearly indicates errors in the predetermined between-stop distances. If you check what you have entered in column (9), you would notice the 7 miles from stop 3 to stop 4 . Correct the data-entry error by replacing 7 by 0.7 . After this change,

(1) vehicle trip length matches the route length,

(2) APTL becomes smaller than the route length, and

(3) PMT is reduced to 34.7 with an APTL of 1.58 miles.

(d) To identify the source of error (3), compare the calculated loads with the observed loads. You may notice that the calculated load is lower by 2 at the first stop. It appears that the ride checker did not include the number of passengers from the previous trip in the number of boardings at the first stop. With this correction,

(1) Boardings become greater than alightings. 
(2) PMT is increased to 42.7 miles with an APTL of 1.78 miles.

Table 67.05. Example of Correcting Data Errors

\begin{tabular}{|c|c|c|c|c|c|c|c|c|}
\hline $\begin{array}{c}\text { Stop } \\
\text { Sequence }\end{array}$ & $\begin{array}{c}\text { Distance } \\
\text { to Next } \\
\text { Stop }\end{array}$ & $\begin{array}{c}\text { No. of } \\
\text { Pass. } \\
\text { Boarded }\end{array}$ & $\begin{array}{c}\text { No. of } \\
\text { Pass. } \\
\text { Alighted }\end{array}$ & $\begin{array}{c}\text { Observed } \\
\text { Leaving } \\
\text { Load }\end{array}$ & $\begin{array}{c}\text { No. of Pass. } \\
\text { from Previous } \\
\text { Trip (Stop 1 } \\
\text { only) }\end{array}$ & $\begin{array}{c}\text { No. of Pass. } \\
\text { Continuing to } \\
\text { Next Trip } \\
\text { Last Stop } \\
\text { only) }\end{array}$ & $\begin{array}{c}\text { Calculated } \\
\text { Leaving } \\
\text { Load }\end{array}$ & PMT \\
\hline$(7)$ & $(9)$ & $(10)$ & $(11)$ & $(12)$ & $(13)$ & $(14)$ & $(15)$ & $(16)$ \\
\hline 1 & 0.0 & 18 & 0 & 20 & 2 & & 18 & 0.0 \\
\hline 2 & 0.3 & 2 & 1 & 21 & & & 19 & 5.7 \\
\hline 3 & 7.0 & 0 & 2 & 19 & & & 17 & 119.0 \\
\hline 4 & 0.6 & 1 & 3 & 17 & & & 15 & 9.0 \\
\hline 5 & 0.3 & 1 & 9 & 8 & & & 7 & 2.1 \\
\hline 6 & 0.5 & 0 & 2 & 6 & & & 5 & 2.5 \\
\hline 7 & 0.8 & 0 & 1 & 5 & & & 4 & 3.2 \\
\hline 8 & 0.2 & 0 & 2 & 3 & & & 0 & 0.4 \\
\hline 9 & 0.1 & 0 & 2 & 1 & & & 0 & 0.0 \\
\hline 10 & 0.1 & 0 & 0 & 1 & & & 0 & 0.0 \\
\hline 11 & 0.3 & 0 & 0 & 1 & & 1 & -1 & -0.1 \\
\hline 12 & 0.1 & 0 & 1 & 1 & & & N/A & 141.8 \\
\hline Total & 10.3 & 22 & 23 & & 2 & 1 & & \\
\hline
\end{tabular}

(e) To identify why boardings are still larger than alightings, compare the recalculated loads with the observed loads again. You may notice that the recalculated load starts deviating from the observed load at stop 5. Since the calculated load is one passenger too big, increase the number of alighted passengers at stop 5 by 1 from 9 to 10 . With this correction,

(1) Boardings equal alightings,

(2) The leaving load at the end stop is 0 , and

(3) PMT is further reduced to 40.3 with an APTL of 1.68 miles.

(f) You should also check the consistency between between-stop distances and the calculated loads. Column (9) is based on the distance from the previous stop, but the calculated load is the leaving load. Correcting this error leads to a final PMT of 47.8 with an APTL of 1.99 miles.

(g) Table 67.07 also shows vehicle trip distance, UPT, PMT, APTL, and ratio PMT/PPMT for the cumulative corrections in (c), (d), (e), and (f).

Table 67.07. Impacts of Correcting Errors

\begin{tabular}{|l|c|c|c|c|c|}
\hline Error Corrections & Vehicle Trip Length & PMT & UPT & APTL & PMT/PPMT \\
\hline No corrections & 10.3 & 141.8 & 22 & 6.45 & 0.63 \\
\hline$\S 69.13(\mathrm{c})$ & 4.0 & 34.7 & 22 & 1.58 & 0.39 \\
\hline$\S 69.13(\mathrm{c})+(\mathrm{d})$ & 4.0 & 42.7 & 24 & 1.78 & 0.44 \\
\hline
\end{tabular}




\begin{tabular}{|l|l|l|l|l|l|}
\hline$\S 69.13(\mathrm{c})+(\mathrm{d})+(\mathrm{e})$ & 4.0 & 40.3 & 24 & 1.68 & 0.42 \\
\hline$\S 69.13(\mathrm{c})+(\mathrm{d})+(\mathrm{e})+(\mathrm{f})$ & 4.0 & 47.8 & 24 & 1.99 & 0.50 \\
\hline
\end{tabular}

\$ 67.15 What should I do if I fail to correct the data errors for a particular service unit?

(a) You should treat the service unit as if it were missed for data collection.

(b) You should follow the guidance in $\$ 65.29$ for getting a replacement unit.

§ 67.17 What steps should I take after I have identified and corrected errors in my data?

(a) You should stack the corrected sample data from the field as shown in Table 67.01 in one or more worksheets. These worksheets along with the survey sheets become an auditable record of your sample data.

(b) You should enter the summary data at the level of your unit of sampling and measurement in a separate worksheet. These summary data are ready for developing new sampling plans or for estimating service-consumed data for the NTD.

(c) Table 67.09 shows how that summary worksheet looks with the column headings and the summary sample data for one service unit.

Table 67.09. Example of Summary Sample Data

\begin{tabular}{|c|c|c|c|c|c|c|c|c|}
\hline Date & $\begin{array}{c}\text { Day of } \\
\text { Week }\end{array}$ & $\begin{array}{c}\text { Time } \\
\text { Period }\end{array}$ & $\begin{array}{c}\text { Route } \\
\text { No. }\end{array}$ & $\begin{array}{c}\text { Trip } \\
\text { No. }\end{array}$ & Direction & $\begin{array}{c}\text { Vehicle Trip } \\
\text { Length }\end{array}$ & UPT & PMT \\
\hline$(1)$ & $(2)$ & $(3)$ & $(4)$ & $(5)$ & $(6)$ & & & \\
\hline $10 / 13 / 05$ & Thur & Midday & 11 & 408 & Outbound & 4.0 & 24 & 47.8 \\
\hline
\end{tabular}




\section{SECTION 70 - ESTIMATION WITHOUT 100\% UPT}

\section{Subsection 71 - General Directions}

\section{\$ 71.01 What service-consumed data must I estimate without 100\% UPT?}

(a) You must estimate both UPT and PMT.

(b) The following table shows the data items you must estimate.

Table 71.01. Service-Consumed Data Items without $100 \%$ UPT

\begin{tabular}{|c|c|}
\hline If your mode is & you must estimate \\
\hline demand response - taxi (DT) & - annual total UPT and PMT \\
\hline $\begin{array}{l}\text { commuter rail (CR), heavy rail } \\
\text { (HR), or light rail (CR) }\end{array}$ & $\begin{array}{l}\text { - annual total UPT and PMT } \\
\text { - average daily UPT and PMT by type of service days } \\
\text { - annual total UPT by weekday time period }\end{array}$ \\
\hline any other mode & $\begin{array}{l}\text { - annual total UPT and PMT } \\
\text { - average daily UPT and PMT by type of service days }\end{array}$ \\
\hline
\end{tabular}

\section{$\$ 71.03$ What do I need to do in general to get estimates of these data items?}

(a) You must determine sample averages.

(b) You must determine expansion factors.

(c) You must combine the expansion factors and sample averages to get the corresponding estimates of service-consumed data.

\section{$\$ 71.05 \quad$ What is a sample average?}

(a) A sample average is the sample total divided by the number of service units in the sample.

(b) It may be calculated for the entire annual sample, by the type of service day, or for specific weekday time periods.

(c) Sample averages are used to estimate service-consumed data when your sampling plan is based on the base option.

\section{\$ 71.07 What is an expansion factor?}

(a) A measure of actual services operated during a given duration of time.

(b) It is used to convert sample averages to totals in the given duration. The total number of one-way bus trips operated during an entire report year is an example of an expansion factor in actual services operated; when multiplied by sample average PMT per one-way bus trip, it yields a measure of annual total PMT.

\section{$\$ 71.09 \quad$ How is the guidance organized?}

(a) The guidance is separate in three subsections for three modal groups to reduce confusion over the different units of sampling and measurement that are typically used for these three modal groups: 
(1) Non-scheduled services, including demand response (DR and DT), vanpool (VP), jitney (JT), or público (PB) (Subsection 73).

(2) Rail services, including heavy rail (HR), commuter rail (CR), light rail (LR), monorail and automated guideway (MG) (Subsection 75).

(3) Bus services, including bus (MB), commuter bus (CB), bus rapid transit (RB), or trolley bus (TB) (Subsection 77).

(b) Subsection 79 contains the guidance on combining expansion factors and sample averages to get estimates of service-consumed data.

\section{Subsection 73 - Non-Scheduled Services}

\section{§ 73.01 What expansion factor should I use for non-scheduled service?}

(a) You should use your 100\% count of vehicle days as the expansion factor.

(b) For estimating annual total PMT and UPT:

(1) Use annual total count of vehicle days if your sampling plan is not based on grouping.

(2) Use annual total count of vehicle days by group if your sampling plan is based on grouping.

(c) For estimating average daily PMT and UPT by type of service days, use annual total count of vehicle days by type of service days.

\section{§ 73.03 What sample average should I use for non-scheduled service?}

(a) You must use the ratio of sample total PMT over sample total vehicle days as the sample average to estimate PMT.

(b) You must use the ratio of sample total UPT over sample total vehicle days as the sample average to estimate UPT.

(c) For estimating annual total PMT and UPT:

(1) Use the sample averages for the entire sample if you do not group your service.

(2) Use the sample averages for each group if your sampling plan is based on grouping.

(d) For estimating average daily PMT and UPT by type of service days, use the sample averages by type of service days. If your sample happens not to include any vehicle-days for a particular day type (e.g., Saturday), use the sample average for the entire sample.

\section{$\S$ 73.05 How do I determine annual vehicle days actually operated for non-scheduled services?}

(a) You should use a spreadsheet or some other mechanism to record the daily number of passengers carried by individual vehicles in your fleet for an entire year.

(b) Figure 73.01 shows an example of such a spreadsheet with the following assumptions:

(1) your fiscal year runs from July 1 through June 30 ,

(2) you operate every day, and

(3) your fleet has 100 vehicles.

(c) Once you have such a spreadsheet, you can easily determine the daily number of vehicles operated by type of service days in two steps:

(1) For any one operating date, count the number of vehicles in the fleet that carried at least one passenger on that date.

(2) Summarize the daily number of vehicles operated by type of service days. 
(d) You can also use such a spreadsheet to determine the number of vehicles operated for each group if your sampling plan is based on service grouping.

\begin{tabular}{|c|c|c|c|c|c|c|c|c|c|c|c|c|}
\hline & $\mathrm{B}$ & C & $\mathrm{D}$ & $E$ & $\mathrm{~F}$ & $\mathrm{G}$ & $\mathrm{H}$ & I & $\mathrm{J}$ & $\ldots$ & $\mathrm{CZ}$ & $\mathrm{DA}$ \\
\hline 1 & \multirow{2}{*}{ Date } & \multirow{2}{*}{ Month } & \multirow{2}{*}{$\begin{array}{l}\text { Day of } \\
\text { Week }\end{array}$} & \multicolumn{9}{|c|}{ Daily Passengers Carried by Vehicle No. } \\
\hline 2 & & & & 1 & 2 & 3 & 4 & 5 & 6 & $\cdots$ & 100 & Total \\
\hline 3 & $7 / 1 / 09$ & July & Wed & & & & & & & & & \\
\hline 4 & $7 / 2 / 09$ & July & Thu & & & & & & & & & \\
\hline 5 & $7 / 3 / 09$ & July & Fri & & & & & & & & & \\
\hline 6 & $7 / 4 / 09$ & July & Sat & & & & & & & & & \\
\hline$\cdots$ & $\cdots$ & $\cdots$ & $\ldots$ & & & & & & & & & \\
\hline 367 & $6 / 30 / 10$ & June & Wed & & & & & & & & & \\
\hline 368 & & Total & & & & & & & & & & \\
\hline
\end{tabular}

Figure 73.01. Daily Passenger Count Worksheet for Non-Scheduled Services

\section{Subsection 75 - Rail Services}

\section{$\$ 75.01 \quad$ What expansion factor should I use for rail services?}

(a) The unit of sampling and measurement you have chosen for your sampling plan determines the expansion factor you should use.

(b) While you could have chosen any unit of sampling measurement for your sampling plan, it most likely is one of the following:

(1) One-way car trips.

(2) One-way train trips.

(3) Round-trip car trips.

(4) Round-trip train trips.

(c) The following assumes that your sampling plan is based on one-way car trips.

(d) For estimating annual total PMT and UPT:

(1) Use annual total one-way car trips if your sampling plan is not based on grouping.

(2) Use annual total one-way car trips by group if your sampling plan is based on grouping.

(e) For estimating average daily PMT and UPT by type of service days, use annual total oneway car trips by type of service days.

(f) For estimating annual total UPT for heavy rail (HR), commuter rail (CR), light rail (LR) by weekday time period, use annual total one-way car trips by weekday time period.

\section{$\$$ 75.03 What sample average should I use for rail services?}

(a) The unit of sampling and measurement you have chosen for your sampling plan determines the sample average you should use.

(b) While you could have chosen any unit of sampling measurement for your sampling plan, it most likely is one of the following:

(1) One-way car trips.

(2) One-way train trips. 
(3) Round-trip car trips.

(4) Round-trip train trips.

(c) Assuming that your unit of sampling and measurement is in one-way car trips, Table 75.01 shows the sample averages you should use.

(d) If your sample happens not to include any one-way car trips for a particular weekday time period, use UPT per one-way car trip for weekdays to estimate the annual total UPT for this weekday time period.

Table 75.01. Sample Averages for Rail Services

\begin{tabular}{|c|c|c|c|c|}
\hline $\begin{array}{l}\text { If the service- } \\
\text { consumed } \\
\text { measure is }\end{array}$ & $\begin{array}{l}\text { and if the } \\
\text { measure is }\end{array}$ & $\begin{array}{l}\text { and if your } \\
\text { sampling plan is }\end{array}$ & and if your mode is & $\begin{array}{l}\text { then you should calculate } \\
\text { the following sample } \\
\text { average }\end{array}$ \\
\hline \multirow{4}{*}{ UPT } & \multirow{2}{*}{ for annual total } & $\begin{array}{l}\text { not based on } \\
\text { grouping }\end{array}$ & & UPT/one-way car trip \\
\hline & & based on grouping & & $\begin{array}{l}\text { UPT/one-way car trip by } \\
\text { group }\end{array}$ \\
\hline & for daily average & & & $\begin{array}{l}\text { UPT/one-way car trip by } \\
\text { type of service days }\end{array}$ \\
\hline & $\begin{array}{l}\text { by weekday time } \\
\text { period }\end{array}$ & & $\begin{array}{l}\text { commuter rail }(\mathrm{CR}) \text {, } \\
\text { heavy rail }(\mathrm{HR}) \text {, or } \\
\text { light rail }(\mathrm{LR})\end{array}$ & $\begin{array}{l}\text { UPT/one-way car trip by } \\
\text { weekday time period }\end{array}$ \\
\hline \multirow{3}{*}{ PMT } & \multirow{2}{*}{ for annual total } & $\begin{array}{l}\text { not based on } \\
\text { grouping }\end{array}$ & & PMT/one-way car trip \\
\hline & & based on grouping & & $\begin{array}{l}\text { PMT/one-way car trip by } \\
\text { group }\end{array}$ \\
\hline & for daily average & & & $\begin{array}{l}\text { PMT/one-way car trip by } \\
\text { type of service days }\end{array}$ \\
\hline
\end{tabular}

\section{\$ 75.05 What steps should I follow to calculate sample averages?}

(a) Aggregate the field sample data to the individual service units in your sample after you have identified and corrected any errors in the data.

(b) If the unit of sampling and measurement for your sampling plan is one-way car trips, sum these quantities over individual one-way car trips in the sample to get sample totals:

(1) for the entire sample,

(2) by type of service days,

(3) by weekday time period if your service is commuter rail (CR), heavy rail (HR), or light rail (LR), and

(4) by group if your sampling plan is based on service grouping.

(c) Count the number of one-way car trips in the sample:

(1) for the entire sample,

(2) by type of service days,

(3) by weekday time period if your service is commuter rail (CR), heavy rail (HR), or light rail (LR), and

(4) by group if your sampling plan is based on service grouping.

(d) Divide sample totals by the number of one-way car trips for the entire sample, by type of service days, by weekday time period if applicable, or by service group if applicable. 


\section{\$ 75.07 How should I determine annual services actually provided as expansion factors?}

(a) You should measure annual services actually provided in the unit of sampling and measurement you have chosen for your sampling plan.

(b) For estimating annual totals of service-consumed data, you should follow these steps:

(1) Start with your schedule.

(2) When the schedule is changed by policy or by emergency conditions, the count from the schedule must be adjusted accordingly.

(3) You must also include added services such as trippers and other special operations.

(4) If your sampling plan is based on service grouping, you must also count annual services actually provided for each group.

(c) For estimating service-consumed data by type of service days, you should also start your schedule, and exclude scheduled services on atypical days.

(d) For estimating annual total UPT for commuter rail (CR), heavy rail (HR), or light rail (LR) by weekday time period, you should start with your weekday schedule, and exclude scheduled services on atypical weekdays.

(e) Refer to the NTD Reporting Manual on determining what are considered atypical days.

\section{Subsection 77 - Bus Services}

\section{\$ 77.01 What expansion factor should I use for bus services?}

(a) The unit of sampling and measurement you have chosen for your sampling plan determines the expansion factor you should use.

(b) While you could have chosen any unit of sampling measurement for your sampling plan, it most likely is in one-way bus trips or in round-trip bus trips.

(c) The following assumes that your sampling plan is based on one-way bus trips.

(d) For estimating annual total PMT and UPT:

(1) Use annual total one-way bus trips if your sampling plan is not based on grouping.

(2) Use annual total one-way bus trips by group if your sampling plan is based on grouping.

(e) For estimating average daily PMT and UPT by type of service days, use annual total oneway bus trips by type of service days.

\section{§ 77.03 What sample average should I use for bus services?}

(a) The unit of sampling and measurement you have chosen for your sampling plan determines the sample average you should use.

(b) While you could have chosen any unit of sampling measurement for your sampling plan, it most likely is in one-way bus trips or in round-trip bus trips.

(c) Assuming that your unit of sampling and measurement is in one-way bus trips, Table 77.01 shows the sample averages you should use.

(d) If your sample happens not to include any one-way bus trips for a particular day type (e.g., Saturday), use the sample average for the entire sample. 
Table 77.01. Sample Averages for Bus Services

\begin{tabular}{|l|l|l|l|}
\hline $\begin{array}{l}\text { If the } \\
\text { service- } \\
\text { consumed } \\
\text { measure is }\end{array}$ & $\begin{array}{l}\text { and if the measure } \\
\text { is }\end{array}$ & $\begin{array}{l}\text { and if your sampling } \\
\text { plan is }\end{array}$ & $\begin{array}{l}\text { then you should calculate the } \\
\text { following sample average }\end{array}$ \\
\hline \multirow{3}{*}{ UPT } & for annual total & not based on grouping & UPT/one-way car trip \\
\cline { 2 - 4 } & for daily average & & UPT/one-way car trip by group \\
\hline \multirow{3}{*}{ PMT } & for annual total & not based on grouping & $\begin{array}{l}\text { UPT/one-way car trip by type of } \\
\text { service days }\end{array}$ \\
\cline { 2 - 4 } & for daily average & & PMT/one-way car trip \\
\cline { 2 - 4 } & fon grouping & $\begin{array}{l}\text { PMT/one-way car trip by group } \\
\text { sMT/one-way car trip by type of } \\
\text { service days }\end{array}$ \\
\hline
\end{tabular}

\section{§77.05 What steps should I follow to calculate sample averages?}

(a) Aggregate the field sample data to the individual service units in your sample after you have identified and corrected any errors in the data.

(b) If the unit of sampling and measurement for your sampling plan is one-way bus trips, sum these quantities over individual one-way bus trips in the sample to get sample totals:

(1) for the entire sample,

(2) by type of service days,

(3) by group if your sampling plan is based on service grouping.

(c) Count the number of one-way bus trips in the sample:

(1) for the entire sample,

(2) by type of service days,

(3) by group if your sampling plan is based on service grouping.

(d) Divide sample totals by the number of one-way bus trips for the entire sample, by type of service days, or by service group if applicable.

\section{\$77.07 How should I determine annual services actually provided as expansion factors?}

(a) You should measure annual services actually provided in the unit of sampling and measurement you have chosen for your sampling plan.

(b) For estimating annual totals of service-consumed data, you should follow these steps:

(1) Start with your schedule.

(2) When the schedule is changed by policy or by emergency conditions, the count from the schedule must be adjusted accordingly.

(3) You must also include added services such as trippers and other special operations.

(4) If your sampling plan is based on service grouping, you must also count annual services actually provided for each group.

(c) For estimating service-consumed data by type of service days, you should also start with your schedule, and exclude scheduled services on atypical days. Refer to the NTD Reporting Manual for determining what are considered atypical days. 


\section{Subsection 79 - Estimating Service-Consumed Data}

\$ 79.01 How should I estimate the annual total of my service-consumed data?

(a) If your sampling plan is not based on service grouping, you should multiply your sample average for the entire sample with the annual total of your corresponding expansion factor to get an estimate of the annual total.

(b) If your sampling plan is based on service grouping, you should take a two-step approach:

(1) Multiply your sample average with your corresponding expansion factor for each group, and

(2) Sum the above products across all groups to get your annual total.

\section{§79.03 How should I estimate average daily of service-consumed data by type of service days?}

(a) If you operate your service only on weekdays, all you need to do is to divide your estimated annual total by the number of typical weekday days of service.

(b) If you operate your service on Saturdays, Sundays, or both as well, you should take the following two steps:

(1) Multiply your sample average(s) for each type of service days with the corresponding expansion factor to get the annual total for each type of service days.

(2) Divide the annual total for each type of service days by the corresponding number of typical days for each type of service days.

\section{$\S 79.05$ How should I estimate annual total UPT for each weekday period for commuter rail, heavy rail, and light rail?}

(a) For each of the weekday periods, you should simply multiply the sample average UPT you have calculated for that period by the corresponding expansion factor to get an estimate of the annual total UPT for that weekday period. 


\section{SECTION 80 - ESTIMATION WITH 100\% UPT}

\section{Subsection 81 - General Directions}

\section{\$ 81.01 What service-consumed data must I estimate with 100\% UPT?}

(a) You must estimate PMT.

(b) The following table shows the data items you must estimate.

Table 81.01. Service-Consumed Data Items with 100\% UPT

\begin{tabular}{|c|c|}
\hline $\begin{array}{l}\text { If your sampling } \\
\text { plan is based on the }\end{array}$ & you must estimate \\
\hline APTL option & $\begin{array}{l}\text { - annual total PMT } \\
\text { - average daily PMT by type of service days }\end{array}$ \\
\hline PPMT option & $\begin{array}{l}\text { - annual total PMT } \\
\text { - average daily PMT by type of service days }\end{array}$ \\
\hline
\end{tabular}

\section{\$ 81.03 What do I need to do in general to get estimates of these data items?}

(a) You must determine expansion factors.

(b) You must determine sample ratios.

(c) You must combine the expansion factors and sample ratios to get the corresponding estimates of service-consumed data.

\section{\$ 81.05 What is a sample ratio?}

(a) A sample ratio is the ratio between the sample total of one measure of service-consumed and the sample total of another measure of service-consumed. This Sampling Manual uses two sample ratios:

(1) The ratio of sample total PMT over sample total UPT gives the sample APTL.

(2) The ratio of sample total PMT over sample total PPMT gives the sample PMT/PPMT ratio.

(b) They may be calculated for the entire annual sample or by the type of service days.

\$ 81.07 What is an expansion factor when I have 100\% UPT?

(a) A measure of actual services consumed during a given duration of time.

(b) It is used to convert sample ratios to totals in the given duration. A $100 \%$ count of UPT consumed during an entire year is an example of an expansion factor in service consumed; when multiplied by sample APTL, it yields a measure of annual total PMT.

\section{Subsection 83 - APTL Option}

\$ 83.01 What expansion factor should I use for the APTL option?

(a) You must use your $100 \%$ count of UPT as the expansion factor. 
(b) For estimating average daily PMT by type of service days, use your annual total $100 \%$ count of UPT by type of service days.

(c) For estimating annual total PMT:

(1) Use your annual total $100 \%$ count of UPT if your sampling plan is not based on grouping.

(2) Use your annual total $100 \%$ count of UPT by service group if your sampling plan is based on grouping and you have reliable $100 \%$ counts of UPT by service group and have entered information into the template accordingly in developing your current template sampling plan. Refer to cell M52 of the PeriodInput Worksheet in the template you used in developing your current period-based template sampling plan, and it should be 1 .

(3) Use your annual total 100\% count of UPT if your sampling plan is based on grouping but you do not have reliable $100 \%$ counts of UPT by service group and you have entered information into the template accordingly in developing your current periodbased template sampling plan. Refer to cell M52 of the PeriodInput Worksheet in the template you used in developing your current period-based template sampling plan, and it should be 0 .

\section{$\S$ 83.03 What sample ratio should I use for the APTL option?}

(a) You must use the sample APTL as the sample ratio.

(b) Use sample APTL by type of service days for estimating average daily PMT by type of service days. If your sample happens not to include service unit for a particular day type (e.g., Saturday), use the sample APTL for the entire sample for this day type.

(c) For estimating annual total PMT:

(1) Use sample APTL for the entire sample if you do not group your service.

(2) Use sample APTL for each group if your sampling plan is based on grouping and you have reliable $100 \%$ counts of UPT by service group and have entered information into the template accordingly in developing your current period-based template sampling plan. Refer to cell M52 of the PeriodInput Worksheet in the template you used in developing your current period-based template sampling plan, and it should be 1 .

(3) Use weighted sample APTL for the entire sample if your sampling plan is based on grouping but you do not have reliable $100 \%$ counts of UPT by service group and you have entered information into the template accordingly in developing your current period-based template sampling plan. Refer to cell M52 of the PeriodInput Worksheet in the template you used in developing your current period-based template sampling plan, and it should be 0 .

\section{§ 83.05 How should I determine the APTL from my sample?}

(a) You must determine the sample APTL for a given sample as the ratio of sample total PMT over sample total UPT for the following cases:

(1) for the entire sample,

(2) by type of service days, or

(3) by service group if applicable.

(b) You must not determine the sample APTL as the average of the APTL across individual service units in the sample. 
(c) To determine the weighted sample APTL for an entire sample as required by question $\$ 83.03(\mathrm{c})(3)$, you should follow these steps:

(1) Determine each group's size in the number of service units actual operated.

(2) Compute each group's share of the number of service units actually operated. These shares must sum to 1 .

(3) Determine each group's sample size in the number of service units in the sample.

(4) Determine each group's sample total UPT.

(5) Determine each group's sample total PMT.

(6) Compute each group's sample average for UPT.

(7) Compute each group's sample average for PMT.

(8) Sum the product of each group's share of the number of service units actually operated and its sample average UPT.

(9) Sum the product of each group's share of the number of service units actually operated and its sample average PMT.

(10) Compute the ratio of the result from (9) to the result from (8). This ratio gives the weighted sample APTL.

(11) Table 83.01 shows these steps with an example.

Table 83.01. Example of Estimating Weighted Sample APTL

\begin{tabular}{|c|l|c|c|c|}
\hline \multirow{2}{*}{ Step } & \multirow{2}{*}{ Description } & \multicolumn{3}{|c|}{ Service Groups } \\
\cline { 3 - 5 } & & Short Routes & Medium Routes & Long Routes \\
\hline 1 & Group size in service units & 109,685 & 331,033 & 35,325 \\
\hline 2 & Group size in shares & 0.2304 & 0.6954 & 0.0742 \\
\hline 3 & Sample size & 116 & 386 & 47 \\
\hline 4 & Sample total UPT & 1,157 & 8,181 & 1,592 \\
\hline 5 & Sample total PMT & 3,989 & 42,966 & 7,003 \\
\hline 6 & Sample average UPT & 10.0 & 21.2 & 33.9 \\
\hline 7 & Sample average PMT & 34.4 & 111.3 & 149.0 \\
\hline 8 & Weighted sample average UPT & \multicolumn{3}{|c|}{19.55} \\
\hline 9 & Weighted sample average PMT & 96.38 \\
\hline 10 & Weighted sample APTL & 4.93 \\
\hline
\end{tabular}

\section{\$ 83.07 How should I estimate annual total PMT for the APTL option?}

(a) If your sampling plan is not based on service grouping, you should multiply your sample APTL for the entire annual sample with your corresponding annual expansion factor (i.e., $100 \%$ count of annual UPT) to get an estimate of the annual total PMT.

(b) You should take a two-step approach if your sampling plan is based on service grouping and you have reliable $100 \%$ counts of UPT by service group:

(1) Multiply your sample APTL with your corresponding expansion factor for each group, and

(2) Sum the above products across all groups to get your annual total PMT.

(c) If your sampling plan is based on service grouping but you do not have reliable $100 \%$ counts of UPT by service group, you should multiple your weighted sample APTL as computed in question $\$ 83.05$ with your $100 \%$ count of annual UPT. 
(d) If your sampling plan is based on service grouping, refer to cell M52 of the PeriodInput Worksheet in the template you used in developing your template sampling plan for what you have entered into cell M52 on whether you have reliable 100\% count of UPT by service group.

\section{§ 83.09 How should I estimate average daily PMT by type of service days?}

(a) If you operate your service only on weekdays, all you need to do is to divide your estimated annual total PMT by the number of typical weekday days of service.

(b) If you operate your service on Saturdays, Sundays, or both as well, you should take the following two steps:

(1) Multiply your sample average APTL for each type of service days with the corresponding $100 \%$ count of UPT to get the annual total PMT for each type of service days.

(2) Divide the estimated annual total PMT for each type of service days by the corresponding number of typical days for each type of service days.

\section{Subsection 85 - PPMT Option}

\section{§ 85.01 What expansion factor should I use for the PPMT option?}

(a) You must use your $100 \%$ count of PPMT as the expansion factor.

(b) For estimating annual total PMT:

(1) Use annual total PPMT if your sampling plan is not based on grouping.

(2) Use annual total PPMT by group if your sampling plan is based on grouping.

(c) For estimating average daily PMT by type of service days, use annual total PPMT by type of service days.

\section{§ 85.03 How do I determine annual total PPMT?}

(a) Suppose that:

(1) Your unit of sampling and measurement is one-way bus trips, and

(2) You are going to estimate annual total PMT.

(b) You should do the following for each route:

(1) Determine the annual number of vehicle revenue miles for the report year.

(2) Determine the annual number of vehicle revenue one-way trips for the report year.

(3) Divide the annual number of vehicle revenue miles by the annual number of vehicle revenue one-way trips to get the average route length.

(4) Multiply your route-level $100 \%$ counts of UPT with the calculated average route length to get route-level PPMT.

(c) You should sum the calculated route-level PPMT across all routes to get your annual PPMT for all routes.

(d) Table 85.01 illustrates how you may accomplish (b) and (c). 
Table 85.01. Calculating Annual Total PPMT for All Operating Routes

\begin{tabular}{|c|l|r|r|r|r|r|}
\hline $\begin{array}{c}\text { Route } \\
\text { Number }\end{array}$ & Route Name & $\begin{array}{r}\text { Annual } \\
\text { Revenue } \\
\text { Trips }\end{array}$ & $\begin{array}{r}\text { Annual } \\
\text { Revenue } \\
\text { Miles }\end{array}$ & $\begin{array}{r}\text { Average } \\
\text { Route } \\
\text { Length }\end{array}$ & $\begin{array}{r}100 \% \\
\text { UPT }\end{array}$ & $\begin{array}{r}100 \% \\
\text { PPMT }\end{array}$ \\
\hline 90 & Blue Line & 3,869 & 9,975 & 2.58 & 22,866 & 58,952 \\
\hline 50 & Red Line & 3,286 & 10,310 & 3.14 & 23,634 & 74,148 \\
\hline 14 & Prospect & 1,643 & 10,690 & 6.51 & 24,506 & 159,446 \\
\hline 12 & Beechcrest & 1,643 & 11,835 & 7.20 & 27,131 & 195,435 \\
\hline 17 & College & 3,286 & 30,666 & 9.33 & 70,298 & 656,036 \\
\hline 37 & Park 100 & 1,325 & 22,733 & 17.16 & 52,112 & 894,068 \\
\hline 8 & Washington & 3,392 & 61,077 & 18.01 & 140,012 & $2,521,072$ \\
\hline 19 & Castleton & 1,696 & 32,916 & 19.41 & 75,457 & $1,464,491$ \\
\hline 26 & Keystone & 1,378 & 28,505 & 20.69 & 65,344 & $1,351,666$ \\
\hline 10 & 10 th St. & 3,339 & 69,897 & 20.93 & 160,231 & $3,354,198$ \\
\hline Total & & & & & $10,729,514$ \\
\hline
\end{tabular}

(e) If your sampling plan is based on service grouping, you must also determine PPMT for each group. Suppose, for example, that you have grouped your short routes into one group and your longer routes into another. Table 85.03 illustrates how you may use route-level information on annual revenue miles, annual revenue trips, and annual UPT to determine PPMT for each group.

Table 85.03. Calculating Annual Total PPMT by Route Group

\begin{tabular}{|c|c|c|c|c|c|c|}
\hline $\begin{array}{l}\text { Route } \\
\text { Group }\end{array}$ & $\begin{array}{l}\text { Route } \\
\text { Number }\end{array}$ & $\begin{array}{r}\text { Annual } \\
\text { Revenue } \\
\text { Trips }\end{array}$ & $\begin{array}{r}\text { Annual } \\
\text { Revenue } \\
\text { Miles }\end{array}$ & $\begin{array}{r}\text { Average } \\
\text { Route } \\
\text { Length }\end{array}$ & $\begin{array}{r}100 \% \\
\text { UPT }\end{array}$ & $\begin{array}{c}100 \% \\
\text { PPMT }\end{array}$ \\
\hline \multirow{6}{*}{ 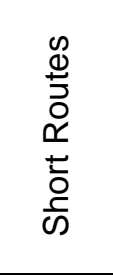 } & 90 & 3,869 & 9,975 & 2.58 & 22,866 & 58,952 \\
\hline & 50 & 3,286 & 10,310 & 3.14 & 23,634 & 74,148 \\
\hline & 14 & 1,643 & 10,690 & 6.51 & 24,506 & 159,446 \\
\hline & 12 & 1,643 & 11,835 & 7.20 & 27,131 & 195,435 \\
\hline & 17 & 3,286 & 30,666 & 9.33 & 70,298 & 656,036 \\
\hline & Total & \multicolumn{5}{|r|}{$1,144,018$} \\
\hline \multirow{6}{*}{ 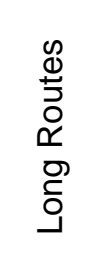 } & 37 & 1,325 & 22,733 & 17.16 & 52,112 & 894,068 \\
\hline & 8 & 3,392 & 61,077 & 18.01 & 140,012 & $2,521,072$ \\
\hline & 19 & 1,696 & 32,916 & 19.41 & 75,457 & $1,464,491$ \\
\hline & 26 & 1,378 & 28,505 & 20.69 & 65,344 & $1,351,666$ \\
\hline & 10 & 3,339 & 69,897 & 20.93 & 160,231 & $3,354,198$ \\
\hline & Total & & & & & $9,585,496$ \\
\hline
\end{tabular}

\section{\$ 85.05 How should I get the sample total of PPMT for each service unit?}

(a) You should follow the guidance for question $\S 85.03$ to determine the average route length for each route. 
(b) Identify the route for each one-way trip in your service units in the sample.

(c) Multiply the sample UPT by the average route length for each one-way trip to get sample PPMT for each one-way trip.

(d) Sum the trip-level PPMT for all one-way trips in a service unit to get sample PPMT for each service unit.

\section{\$ 85.07 What sample ratio should I use for the PPMT option?}

(a) You must use the ratio of sample total PMT over sample total PPMT as the sample ratio.

(b) For estimating annual total PMT:

(1) Use sample PMT/PPMT ratio for the entire sample if you do not group your service.

(2) Use sample PMT/PPMT for each group if your sampling plan is based on grouping.

(c) For estimating average daily PMT by type of service days, use the sample PMT/PPMT ratio by type of service days.

\$ 85.09 How should I determine the PMT/PPMT ratio for a sample?

(a) You must determine the sample PMT/PPMT ratio for a given sample as the ratio of sample total PMT over sample total PPMT.

(b) You must not determine the sample ratio as the average of the PMT/PPMT ratio for individual service units in the sample.

\section{\$ 85.11 How should I estimate annual total PMT for the PPMT option?}

(a) You should multiply your sample PMT/PPMT ratio for the entire annual sample with the annual total of your PPMT to get an estimate of the annual total PMT if your sampling plan is not based on service grouping, or

(b) You should take a two-step approach if your sampling plan is based on service grouping:

(1) Multiply your sample PMT/PPMT ratio with your corresponding annual total PPMT for each group, and

(2) Sum the above products across all groups to get your annual total PMT.

\section{§ 85.13 How should I estimate average daily PMT by type of service days?}

(a) If you operate your service only on weekdays, all you need to do is to divide your estimated annual total PMT by the number of typical weekday days of service.

(b) If you operate your service on Saturdays, Sundays, or both as well, you should take the following two steps:

(1) Multiply your sample PMT/PPMT ratio for each type of service days with the corresponding annual total PPMT to get the annual total PMT for each type of service days.

(2) Divide the annual total PMT for each type of service days by the corresponding number of typical days for each type of service days. 


\section{SECTION 90 - APPENDIXES}

\section{Appendix 91 - Definitions}

\section{\$91.01 What do the sampling-related terms mean?}

Alternative sampling plan. A sampling plan that reflects the conditions of your service, and is independently developed and certified by a qualified statistician to meet FTA's 95\% confidence and $10 \%$ precision levels. It is one of two forms of customized sampling plans. The other form is template sampling plans. It is equivalent to an alternative sampling technique as defined in the NTD Reporting Manual.

Confidence level. The chance of an estimate of service-consumed data obtained through random sampling falling within a particular range of the true value. FTA requires a minimum level of 95\% confidence for estimates of annual UPT and annual PMT reported to the NTD. A particular confidence level is only meaningful when it is stated with a particular precision level.

Customized sampling plan. A sampling plan that reflects the conditions of your service and meets FTA's 95\% confidence and 10\% precision levels. It is either a template sampling plan or an alternative sampling plan. It differs from a ready-to-use sampling plan in that it takes account of the specific characteristics of your service.

Efficiency option. A characteristic of a sampling plan that affects its sampling efficiency.

Initial annual sample size. The annual necessary sample size of a period-based template sampling plan that is determined from the companion spreadsheet template before it is allocated to each quarter, month, or week. This can differ from the realized annual sample size for a given set of sample data.

Mandatory revising year. A report year for which you must consider whether you need to revise your template sampling plan.

Margin of safety. A percent increase in the statistical variation of your sample data in developing a template sampling plan. For example, if the statistical variation of your sample is $S$ and the margin of safety is $25 \%$, you must use $1.25 S$ as the statistical variation in developing your template sampling plan. A margin of safety of $25 \%$ is used automatically for all period-based template sampling plans. If you develop alternative sampling plans, you should also use this margin of safety. The objective is to counter the potential fluctuations in the statistical variation in a sample from one year to another due to sampling and other reasons.

Major change to a service. Any change to your service that is likely to lead to major changes in how your customers use your service. Examples of major changes include making transfers fare free; adding or cutting express routes; expanding or contracting your service by 
more than $25 \%$ in vehicle revenue miles; or restructuring your service affecting more than $25 \%$ of your service in vehicle revenue miles.

Necessary sample size. The sample size that meets FTA's minimum 95\% confidence and $10 \%$ precision levels and uses a $25 \%$ margin of safety.

Precision index. A number that reflects the level of precision that your current NTD sample achieves in the resulting annual total PMT. It is used for you to determine whether you must revise your current template sampling plan after you have made major changes to your service since you started using the current template sampling plan. Once you have entered your current sample data into the template for this Sampling Manual as if you are going to develop new template sampling plans, this precision index is made available in the PeriodPrecision Worksheet for period- based sampling and in the IntervalPrecision Worksheet for interval-based sampling.

Precision level. The degree of errors in an estimate of service-consumed data obtained through random sampling that is stated in percentage terms relative to the true value. FTA requires a minimum of $10 \%$ precision for estimates of annual service-consumed data reported to the NTD. A particular precision level is only meaningful if it is stated with a particular confidence level.

Random sampling. Selection of one or more service units at random from a list of service units to be operated.

Ready-to-use sampling plan. A sampling plan that has been developed specifically for this Sampling Manual with sample data from a variety of transit agencies. It does not necessarily reflect the conditions of your service. Ready-to-use sampling plans have limited applicability.

Realized annual sample size. The annual necessary sample size of a period-based template sampling plan that is based on quarterly, monthly, or weekly sampling. For example, if you choose weekly sampling and your template sampling plan requires 3 one-way trips per week, the realized annual sample size would be 156 one-way trips.

Sample size. The number of service units that are sampled, and for which unlinked passenger trips and passenger miles traveled are measured.

Sampling efficiency. The degree to which a sampling plan minimizes the necessary sample size for meeting FTA's confidence and precision levels. Sampling plans that take advantage of certain characteristics of your service can sometimes require a smaller necessary sample size. A smaller necessary sample size reduces the time and cost of sampling, data collection, and data processing.

Sampling frequency. The number of times per year that a sample is drawn; in this Manual, sampling frequency is quarterly, monthly, or weekly for period-based sampling, and is every day, every $2^{\text {nd }}$ day, every $3^{\text {rd }}$ day, every $4^{\text {th }}$ day, every $5^{\text {th }}$ day, and every $6^{\text {th }}$ day for interval- 
based sampling. For example, if your period-based sampling plan requires 10 service units per month, before the current month ends you must select at least 10 at random from the full list of all service units to be operated during the next month.

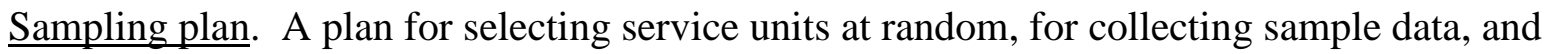
for estimating annual service-consumed data that meets FTA's 95\% confidence and $10 \%$ precision levels. Each sampling plan consists of four elements: a unit of sampling and measurement, a set of efficiency options, a sampling frequency, and a necessary sample size.

Sampling without replacement. Selection of a sample of service units at random without the chance of a single service unit being selected more than once.

Sampling year. Any report year for which you obtained annual UPT, annual PMT, or both through random sampling that meet FTA's $95 \%$ confidence and $10 \%$ precision levels. It can be a mandatory sampling year or an intermediate report year for which you choose to sample.

Service grouping. One efficiency option for which you divide your service into two or more groups with the objectives of reducing within-group differences and increasing betweengroup differences. For example, separating your bus routes into express routes and local routes is likely to reduce differences in average passenger trip length across one-way bus trips within each group.

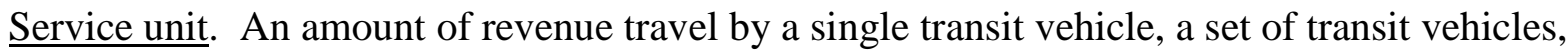
or a component of a transit vehicle. For non-scheduled services, it is typically one vehicle day. For scheduled bus services it is typically either a one-way bus run or else a round-trip bus run. For rail services, it is either a one-way car run, a one-way train run, or a round-trip car run or a round-trip train run.

Statistical variation. The degree of differences in a quantity across the full list of service units operated during a given duration of time, such as differences in PMT across all one-way trips of a bus service in a full report year. A larger variation requires a greater sample size to meet given confidence and precision levels.

Table of random numbers. A list of integers whose frequency and order of appearance in the list have been determined entirely by chance. It is the basis of a commonly used method of random sampling.

Template sampling plan. A sampling plan that is developed with the companion template of this Sampling Manual.

Unit of sampling and measurement. A service unit you choose for your sampling plan.

\$91.03 What do the data-collection terms mean?

Arriving load. The number of passengers onboard a transit vehicle as it arrives at a stop. 
Automatic passenger counter. An automated means of counting passengers as they board or alight transit vehicles with treadle mats, infrared beams, or other devices placed by the doors of a transit vehicle.

Calculated load. The number of passengers onboard a transit vehicle as it arrives at or leaves a stop that you calculate from data on boardings and alightings at individual stops. It should equal the observed load.

Commuter vanpool. A common form of vanpool service (VP) that comprises vans, small buses and other vehicles operating as a ridesharing arrangement, providing transportation to a group of workers commuting directly between their homes and their regular work sites within the same geographical area. The vehicles would not be in revenue service during the working hours of the participating workers.

Distance-based approach. A method to obtain PMT that keeps track of the distance traveled by every passenger.

Leaving load. The number of passengers onboard a transit vehicle as it leaves a stop.

Load-based approach. A method to obtain PMT that is based on the boardings and alightings at individual stops and on the distance between consecutive stops.

NTD sample. The sample of service units you select at random according to your sampling plan that meets FTA's 95\% confidence and 10\% precision levels for reporting to the NTD.

Observed load. The number of passengers onboard a transit vehicle as observed directly by a ride-checker while onboard that transit vehicle.

Ride check. A method of collecting sample data with one or more persons observing and recording passenger boarding and alighting activities while riding in a transit vehicle.

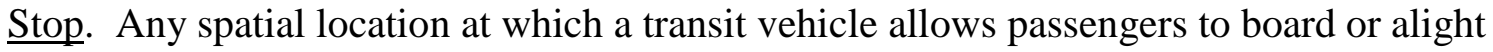
from the vehicle.

Vehicle trip length. The total distance traveled by a transit vehicle during a one-way trip for scheduled services. For example, the cumulative distance traveled from the beginning point to the end point of a particular alignment of a route is the vehicle trip length for this trip. The vehicle trip length may vary by direction and alignment for a given route.

\section{\$91.05 What do the estimation-related terms mean?}

$100 \%$ count. A method of obtaining service-consumed data. This term also often refers to the results of a $100 \%$ count. For UPT, it involves counting passengers each time they board a transit vehicle in revenue service, such as through a registering farebox. For PMT, it involves recording the distance traveled by all passengers. A 100\% count of PMT is typically only possible for systems that have only two stops, for rail systems that record entry and exit from the system, or for rail systems that rely upon destination-based tickets. 
Average passenger trip length (APTL). The average distance traveled for an unlinked passenger trip. It is calculated as PMT divided by UPT.

Average route length. The average length of a route actually traveled by vehicles in scheduled services. It is calculated by dividing the annual vehicle revenue miles by the number of annual vehicle revenue one-way trips for that route.

Expansion factor. A measure of actual services operated or consumed during a given duration of time. It is used to convert a sample average to the total of service-consumed data for that duration. It varies with sampling plans. The total number of one-way bus trips operated during an entire report year is an example of an expansion factor; when multiplied by the sample average PMT per one-way bus trip derived from annual NTD sample, it yields a measure of annual total PMT.

Passenger miles traveled (PMT). The total distance traveled by all passengers during a given period.

Potential passenger miles traveled. The maximum number of passenger miles that could have been traveled by all passengers along a given fixed route during a year (or some other time duration.) It is calculated by multiplying a 100\% count of UPT times the average route length of that route during that duration.

Sample average. The sample total divided by the number of service units in the sample. It may be calculated for the entire annual sample, or by the type of service day, or for specific weekday time periods. For example, dividing the total PMT in an annual NTD sample by the total number of one-way bus trips in the annual NTD sample gives a sample average PMT.

Sample data. The data collected from a sample of service units according to a sampling plan that meets FTA's 95\% confidence and 10\% precision levels.

Sample ratio. The ratio of the sample total for one measure of service-consumed over the sample total for another measure of service-consumed. For example, the ratio of the sample data for PMT over the sample total for UPT gives the sample APTL. It may be calculated for the entire annual sample, or by the type of service days, or for individual service group if your sampling plan is based on service grouping.

Sample total. The sum total of all data across the service units in a random sample. For example, if you are sampling for PMT, the PMT sample total is the sum of the PMT collected for each of the one-way bus trip in the sample. It may be calculated for the entire annual sample, or by the type of service day, or for specific weekday time periods.

Service-consumed data. Passenger miles traveled and unlinked passenger trips.

Type of service days. Weekdays, Saturdays, or Sundays. For scheduled services, service days in a report year are classified according to the schedule operated on that day. If a 
weekday that is a holiday is served with a Sunday schedule, that weekday is considered to be a Sunday. For non-scheduled services, service days are the actual days of a week regardless of whether they are a holiday or not.

Typical day. For your scheduled services, it is a day on which you operate your normal, regular schedule and there are no anomalies such as extra service added for a special event or reduced service as a result of weather or interruption. For your non-scheduled services, it is any day of operation.

Unlinked passenger trips (UPT). The number of passengers who board transit vehicles in revenue service. Passengers are counted each time they board a vehicle, no matter how many vehicles they use to travel from their origin to their destination.

Weekday time periods. Weekday AM Peak, Weekday Midday, Weekday PM Peak, and Weekday Other. The Reporting Manual instructs how you should define the start and end points of each period.

\section{\$ 91.07 What do the reporting terms mean?}

Auditable record. Documentation of information collected and processes used in collecting that information that demonstrates your compliance with NTD requirements. Such documentation may also help quality control within your agency when your NTD staff change over time.

First-time reporting. Reporting of a particular service to the NTD for the first time.

\section{\$ 91.09 What abbreviations for general terms are used in this Manual?}

APTL. Average passenger trip length in miles

APC. Automatic passenger counter

FTA. Federal Transit Administration

NTD. National Transit Database

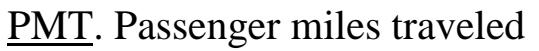

$\underline{\text { PPMT. }}$ Potential passenger miles traveled

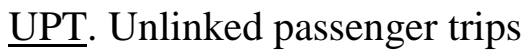

\section{§91.11 What abbreviations for NTD modes are used in this Manual?}

$\underline{\mathrm{CB}}$. Commuter bus - Fixed-route bus systems that are primarily connecting outlying areas with a central city. Service typically uses over-the-road buses with service predominantly in one direction during peak periods, limited stops, and routes of extended length. 
CC. Cable car - A railway propelled by moving cables located beneath the street. While popular at the turn of the last century, the only surviving system is operated in San Francisco.

$\underline{\mathrm{CR}}$. Commuter rail - Rail service operating on either old freight railways, or on tracks that are shared with freight railways, Amtrak, or both. The service is characterized by relatively long distances between stops, for service primarily connecting a central city with outlying suburbs and cities. The service may be either diesel or electric-powered and usually has grade-crossings with roadways.

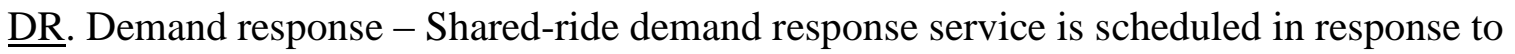
calls from passengers. Many transit systems operate demand response (DR) service to meet the requirements of the Americans with Disabilities Act (ADA).

DT. Demand response - Taxi - A special form of the demand response mode operated through taxicab providers. The mode is always purchased transportation type of service.

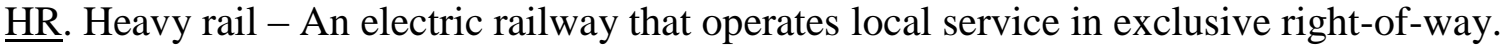
The service is characterized by long trains of six to eight cars or more and by relatively short distances between stops for local service within a city and the immediate suburbs. The Nation's traditional subway systems are classified as heavy rail.

JT. Jitney - A transit mode comprising of owner-operated passenger cars or vans operating on fixed routes (sometimes with minor deviations) as demand warrants without fixed schedules or fixed stops.

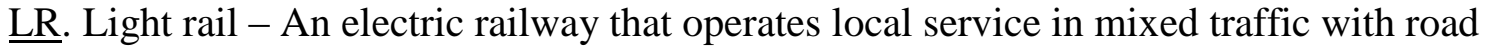
vehicles, or has grade crossings with roadways. The service is characterized by short trains of one to four cars and by relatively short distances between stops for local service within a city and the immediate suburbs.

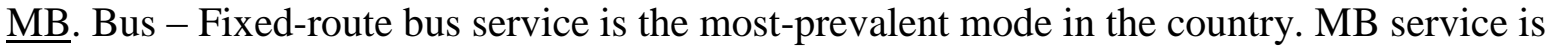
powered by a motor and fuel contained within a vehicle. Deviated fixed-route service is also reported as MB.

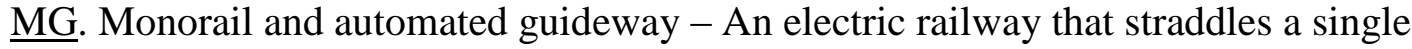
guideway. It may have vehicle operators or may use computers to guide the vehicles.

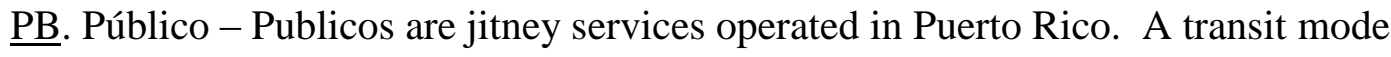
comprising of passenger vans or small buses operating with fixed routes but no fixed schedules. Publicos (PB) are a privately owned and operated public transit service which is market oriented and unsubsidized, but regulated through a public service commission, state or local government. Publicos (PB) are operated under franchise agreements, fares are regulated by route and there are special insurance requirements. Vehicle capacity varies from eight to 24, and the vehicles may be owned or leased by the operator. 
$\underline{\mathrm{RB}}$. Bus rapid transit - Fixed-route bus systems that either (1) operate their routes predominantly on fixed-guideways (other than on highway high occupancy vehicle (HOV) or shoulder lanes, such as for commuter bus service) or (2) that operate routes of highfrequency service with the following elements: Substantial transit stations, traffic signal priority or preemption, low-floor vehicles or level-platform boarding, and separate branding of the service. High-frequency service is defined as 10-minute peak and 15-minute off-peak headways for at least 14 hours of service operations per day. This mode may include portions of service that are fixed-guideway and non-fixed-guideway.

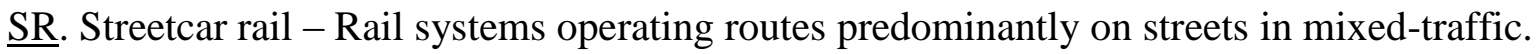
This service typically operates with single-car trains powered by overhead catenaries and with frequent stops.

$\underline{\text { TB}}$. Trolleybus - Fixed-route service using rubber tire buses powered by electric current from overhead wires using trolley poles. Service using rubber tire replica trolleys or historic trolleys, powered by an on-board motor are not included in this mode.

TR. Arial tramway - A system of aerial cables with suspended vehicles.

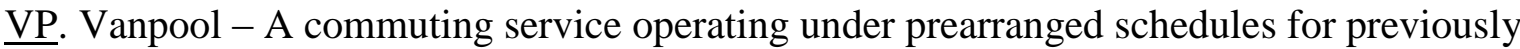
formed groups of riders in vans.

YR. Hybrid rail - Rail systems primarily operating routes on the National system of railroads, but not operating with the characteristics of commuter rail. This service typically operates light rail-type vehicles as diesel multiple-unit trains (DMU's). 


\section{Appendix 92 - Distance-Based Survey Sheet}

\section{Distance-Based Survey Sheet}

(1) Survey Date

(3) Survey Vehicle No.
(2) Day of Week

(4) Driver No.

\begin{tabular}{|c|c|c|c|c|}
\hline$(5)$ & $(6)$ & $(7)$ & $(8)$ & $(9)$ \\
\hline $\begin{array}{c}\text { No. of } \\
\text { Pass. }\end{array}$ & Pick-UP Location & $\begin{array}{c}\text { Pick-UP } \\
\text { Odometer } \\
\text { Reading }\end{array}$ & Drop-Off Location & $\begin{array}{c}\text { Drop-Off } \\
\text { Odometer } \\
\text { Reading }\end{array}$ \\
\hline
\end{tabular}

Page 


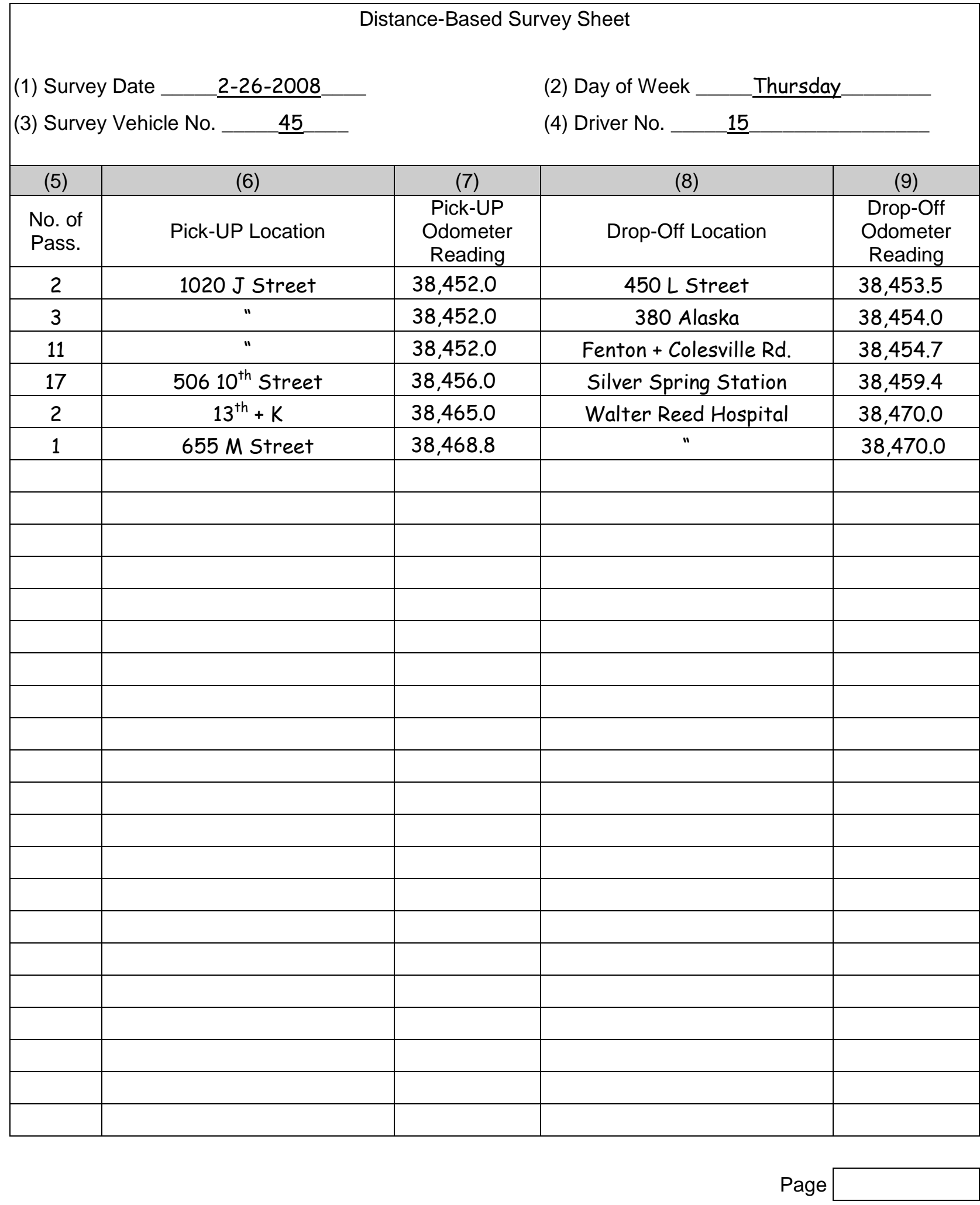




\section{Appendix 93 - Load-Based Survey Sheet for Demand Response (DR or DT)}

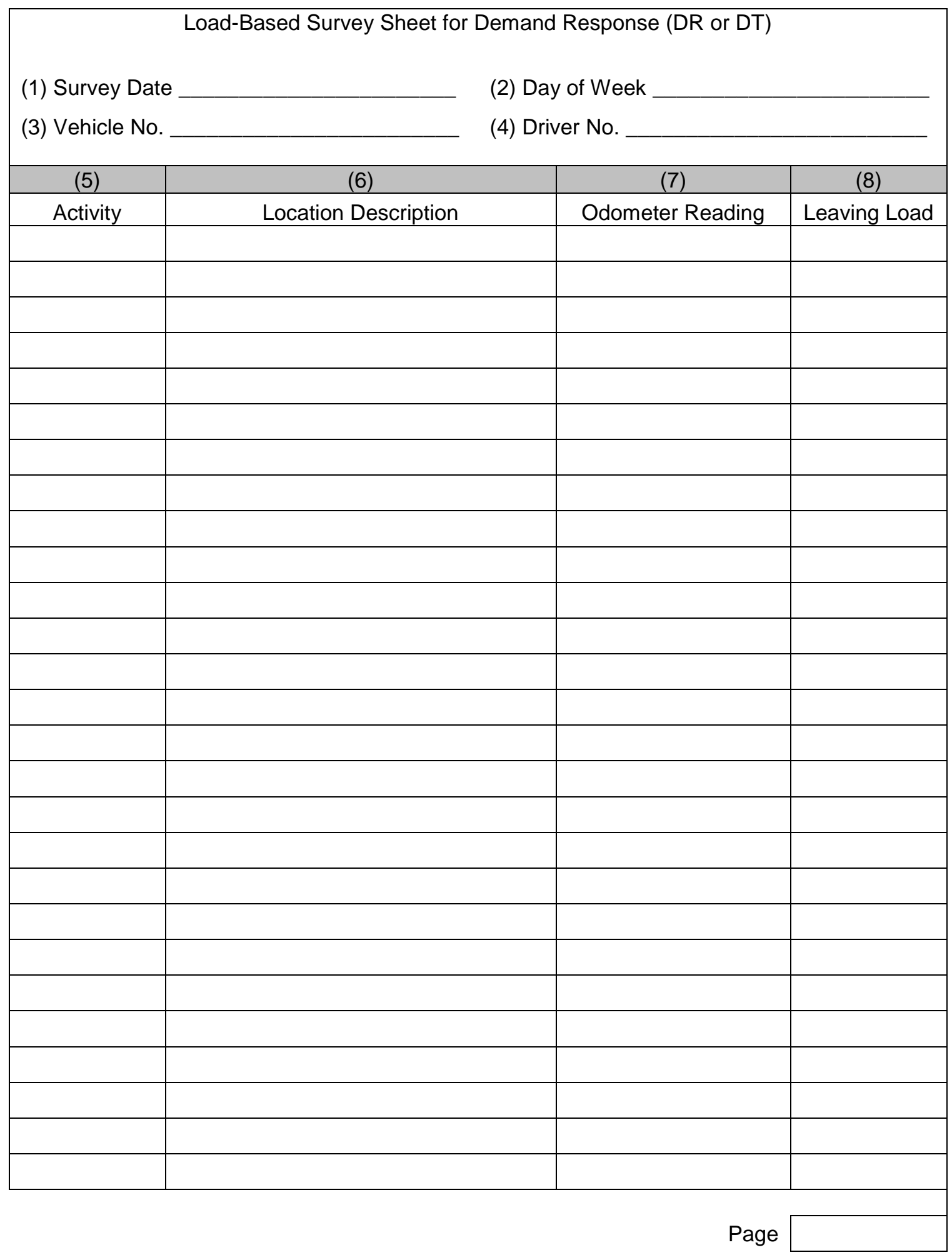




\begin{tabular}{|c|c|c|c|}
\hline \multicolumn{4}{|c|}{ Load-Based Survey Sheet for Demand Response (DR or DT) } \\
\hline \multicolumn{2}{|c|}{ (1) Survey Date $\quad 2 / 26 / 2008$} & (2) Day of Week _ Th & Thursday \\
\hline (3) Vehicle No. & 54 & \multicolumn{2}{|l|}{ (4) Driver No. _ 15} \\
\hline (5) & (6) & (7) & (8) \\
\hline Activity & Location Description & Odometer Readin & Leaving Load \\
\hline Pick-up & $1020 \mathrm{~J}$ Street & $38,452.0$ & 16 \\
\hline Drop-off & 450 L Street & $38,453.5$ & 14 \\
\hline Drop-off & 380 Alaska & $38,454.0$ & 11 \\
\hline Drop-off & Fenton + Colesville Rd & $38,454.7$ & 0 \\
\hline Pick-up & 506 10th Street & $38,456.0$ & 17 \\
\hline Drop-off & Silver Spring Station & $38,459.4$ & 0 \\
\hline Pick-up & 13 th $+K$ & $38,465.0$ & 2 \\
\hline Pick-up & 655 M Street & $38,468.6$ & 3 \\
\hline Drop-off & Walter Reed Hospital & $38,470.0$ & 0 \\
\hline & & & \\
\hline & & & \\
\hline & & & \\
\hline & & & \\
\hline & & & \\
\hline & & & \\
\hline & & & \\
\hline & & & \\
\hline & & & \\
\hline & & & \\
\hline & & & \\
\hline & & & \\
\hline & & & \\
\hline & & & \\
\hline & & & \\
\hline & & & \\
\hline & & & \\
\hline & & & \\
\hline & & $\mathrm{Pa}$ & \\
\hline
\end{tabular}




\section{Appendix 94 - Load-Based Survey Sheet for Commuter Vanpool}

\begin{tabular}{|c|c|c|c|c|}
\hline \multicolumn{5}{|c|}{ Load-Based SURVEY SHEET for Commuter Vanpool } \\
\hline \multicolumn{5}{|c|}{ (1) Date __ (2) Day of Week___ (3) Vanpool No. ___ } \\
\hline (4) & $(5)$ & (6) & (7) & (8) \\
\hline 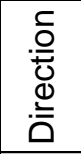 & Pickup/Drop-off & $\begin{array}{c}\text { \#of people } \\
\text { who got ON the Van }\end{array}$ & $\begin{array}{c}\text { \# of people } \\
\text { who got OFF the Van }\end{array}$ & $\begin{array}{c}\text { Van odometer reading } \\
\text { when people got ON/OFF } \\
\text { the Van }\end{array}$ \\
\hline \multirow{14}{*}{ 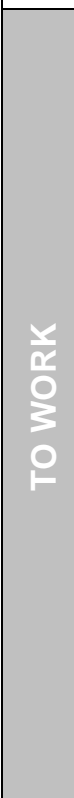 } & Van Starts & & & \\
\hline & 1st Pick-up & & & \\
\hline & 2nd Pick-up & & & \\
\hline & 3rd Pick-up & & & \\
\hline & 4th Pick-up & & & \\
\hline & 5th Pick-up & & & \\
\hline & 6th Pick-up & & & \\
\hline & 1st Drop-off & & & \\
\hline & 2nd Drop-off & & & \\
\hline & 3rd Drop-off & & & \\
\hline & 4th Drop-off & & & \\
\hline & 5th Drop-off & & & \\
\hline & 6th Drop-off & & & \\
\hline & Van Parks & & & \\
\hline \multirow{14}{*}{$\begin{array}{l}\frac{y}{0} \\
\frac{1}{0} \\
3 \\
\frac{1}{0} \\
\frac{0}{11}\end{array}$} & Van Starts & & & \\
\hline & 1st Pick-up & & & \\
\hline & 2nd Pick-up & & & \\
\hline & 3rd Pick-up & & & \\
\hline & 4th Pick-up & & & \\
\hline & 5th Pick-up & & & \\
\hline & 6th Pick-up & & & \\
\hline & 1st Drop-off & & & \\
\hline & 2nd Drop-off & & & \\
\hline & 3rd Drop-off & & & \\
\hline & 4th Drop-off & & & \\
\hline & 5th Drop-off & & & \\
\hline & 6th Drop-off & & & \\
\hline & Van Parks & & & \\
\hline
\end{tabular}




\begin{tabular}{|c|c|c|c|c|}
\hline \multicolumn{5}{|c|}{ Load-Based SURVEY SHEET for Commuter Vanpool } \\
\hline \multicolumn{2}{|r|}{$\underline{2-26-2008}$} & \multirow{2}{*}{ _ (2) Day of Week } & Thursday (3) Vanpool No. & $\underline{28706}$ \\
\hline (4) & $(5)$ & & (7) & $(8)$ \\
\hline $\begin{array}{l}\text { 음 } \\
\stackrel{0}{0} \\
\stackrel{0}{\overline{0}}\end{array}$ & Pickup/Drop-off & $\begin{array}{c}\text { \#of people } \\
\text { who got ON the Van }\end{array}$ & $\begin{array}{c}\text { \# of people } \\
\text { who got OFF the Van }\end{array}$ & $\begin{array}{c}\text { Van odometer reading } \\
\text { when people got ON/OFF } \\
\text { the Van }\end{array}$ \\
\hline \multirow{14}{*}{$\begin{array}{l}\frac{1}{2} \\
\frac{11}{0} \\
3 \\
0\end{array}$} & Van Starts & 1 & & $29,366.0$ \\
\hline & 1st Pick-up & 5 & & 369.1 \\
\hline & 2nd Pick-up & & & \\
\hline & 3rd Pick-up & & & \\
\hline & 4th Pick-up & & & \\
\hline & 5th Pick-up & & & \\
\hline & 6th Pick-up & & & \\
\hline & 1st Drop-off & & 3 & 395.3 \\
\hline & 2nd Drop-off & & 1 & 396.8 \\
\hline & 3rd Drop-off & & 1 & 397.5 \\
\hline & 4th Drop-off & & & \\
\hline & 5th Drop-off & & & \\
\hline & 6th Drop-off & & & \\
\hline & Van Parks & & 1 & 405.0 \\
\hline \multirow{14}{*}{ 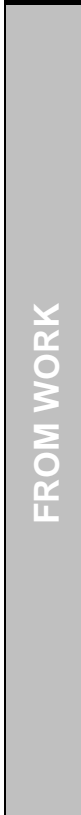 } & Van Starts & 1 & & $29,405.0$ \\
\hline & 1st Pick-up & & & \\
\hline & 2nd Pick-up & & & \\
\hline & 3rd Pick-up & & & \\
\hline & 4th Pick-up & 1 & & 412.5 \\
\hline & 5th Pick-up & 1 & & 413.2 \\
\hline & 6th Pick-up & 3 & & 414.7 \\
\hline & 1st Drop-off & & & \\
\hline & 2nd Drop-off & & & \\
\hline & 3rd Drop-off & & & \\
\hline & 4th Drop-off & & & \\
\hline & 5th Drop-off & & & \\
\hline & 6th Drop-off & & 5 & 440.9 \\
\hline & Van Parks & & 1 & 444.0 \\
\hline
\end{tabular}




\section{Appendix 95 - Load-Based Survey Sheet for Fixed-Route Service}

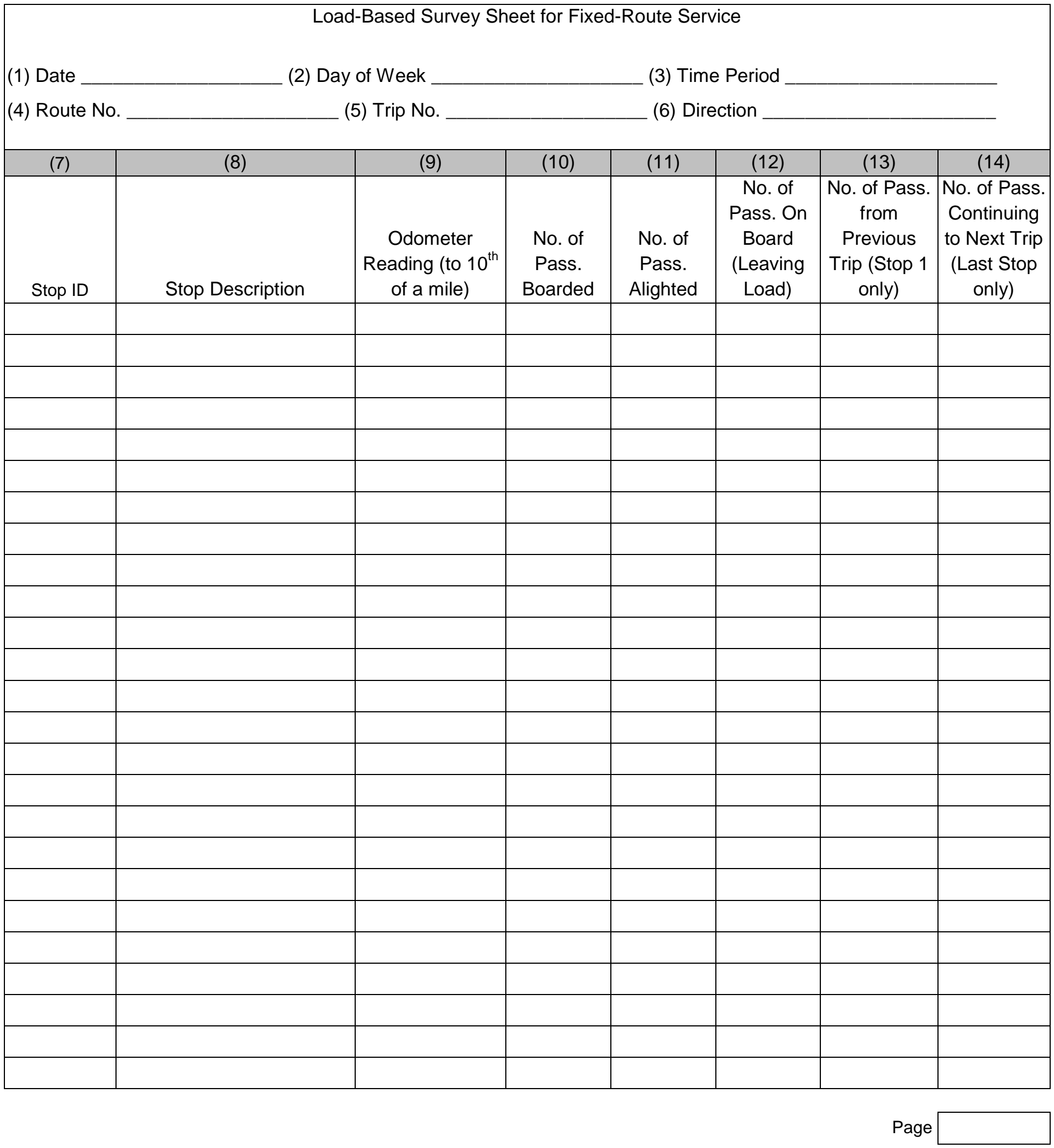


Load-Based Survey Sheet for Fixed-Route Service
(1) Date
(2) Day of Week
Thursday
(3) Time Period
Midday
(4) Route No.
$\underline{11}$
(5) Trip No. $\quad 408$
(6) Direction
Outbound

\begin{tabular}{|c|c|c|c|c|c|c|c|}
\hline$(7)$ & $(8)$ & (9) & $(10)$ & (11) & $(12)$ & (13) & (14) \\
\hline Stop ID & Stop Description & $\begin{array}{c}\text { Odometer } \\
\text { Reading (to } 10 \text { th } \\
\text { of a mile) }\end{array}$ & $\begin{array}{c}\text { No. of } \\
\text { Pass. } \\
\text { Boarded }\end{array}$ & $\begin{array}{c}\text { No. of } \\
\text { Pass. } \\
\text { Alighted }\end{array}$ & $\begin{array}{l}\text { No. of } \\
\text { Pass. On } \\
\text { Board } \\
\text { (Leaving } \\
\text { Load) }\end{array}$ & $\begin{array}{c}\text { No. of Pass. } \\
\text { from } \\
\text { Previous Trip } \\
\text { (Stop } 1 \text { only) }\end{array}$ & $\begin{array}{l}\text { No. of Pass. } \\
\text { Continuing to } \\
\text { Next Trip } \\
\text { (Last Stop } \\
\text { only) }\end{array}$ \\
\hline 1 & Plaza & $41,214.8$ & 20 & 0 & 20 & 2 & \\
\hline 2 & City Hall & 215.1 & 2 & 1 & 21 & & \\
\hline 3 & Bloxham / Adams & 215.8 & 0 & 2 & 19 & & \\
\hline 4 & FAMU \# 1 & 216.4 & 1 & 3 & 17 & & \\
\hline 5 & FAMU \# 2 & 216.7 & 1 & 10 & 8 & & \\
\hline 6 & FAMU \# 3 & 217.2 & 0 & 2 & 6 & & \\
\hline 7 & Lake Bradford / Kissimmee & 218.0 & 0 & 1 & 5 & & \\
\hline 8 & Indian River / Levy & 218.2 & 0 & 2 & 3 & & \\
\hline 9 & Hillsborough / Levy & 218.3 & 0 & 2 & 1 & & \\
\hline 10 & Alumin Village / Levy & 218.4 & 0 & 0 & 1 & & \\
\hline 11 & Iamonia / Levy & 218.7 & 0 & 0 & 1 & & \\
\hline 12 & Iamonia / McCaskill & 218.8 & 0 & 1 & 1 & & 1 \\
\hline & & & & & & & \\
\hline & & & & & & & \\
\hline & & & & & & & \\
\hline & & & & & & & \\
\hline & & & & & & & \\
\hline & & & & & & & \\
\hline & & & & & & & \\
\hline & & & & & & & \\
\hline & & & & & & & \\
\hline & & & & & & & \\
\hline & & & & & & & \\
\hline & & & & & & & \\
\hline & & & & & & & \\
\hline & & & & & & & \\
\hline & & & & & & Page & \\
\hline
\end{tabular}




\section{Appendix 96 - Daily Log Sheet for Commuter Vanpool}

Daily Log Sheet for Commuter Vanpool

(1) Month August 2008

(2) Vanpool No.

\begin{tabular}{|c|c|c|c|}
\hline (3) & (4) & (5) & (6) \\
\hline Date & No. of Riders to Work & No. of Riders from Work & $\begin{array}{c}\text { Total One-Way } \\
\text { Trips }\end{array}$ \\
\hline 1 & $/ / / / / / /$ & $1 / 1 / 1 /$ & 13 \\
\hline 2 & & & \\
\hline 3 & & & \\
\hline 4 & & & \\
\hline 5 & & & \\
\hline 6 & & & \\
\hline 7 & & & \\
\hline 8 & & & \\
\hline 9 & & & \\
\hline 10 & & & \\
\hline 11 & & & \\
\hline 12 & & & \\
\hline 13 & & & \\
\hline 14 & & & \\
\hline 15 & & & \\
\hline 16 & & & \\
\hline 17 & & & \\
\hline 18 & & & \\
\hline 19 & & & \\
\hline 20 & & & \\
\hline 21 & & & \\
\hline 23 & & & \\
\hline 24 & & & \\
\hline 25 & & & \\
\hline 26 & & & \\
\hline 27 & & & \\
\hline 28 & & & \\
\hline 29 & & & \\
\hline 30 & & & \\
\hline 31 & & & \\
\hline
\end{tabular}




\section{Appendix 97 - Full Table of Contents}

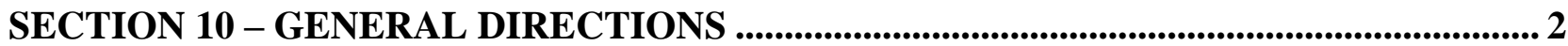

$\S 10.01$ What is the purpose of this Sampling Manual? ....................................................... 2

$\S 10.03$ What procedures does this Sampling Manual cover? ............................................. 2

$\S 10.05$ May I continue using the sampling plans in Circulars 2710.1A and 2710.2A?........... 2

SECTION 30 - ALTRNATIVE SAMPLING PLANS ..................................................................... 3

$\S 30.01$ When should I consider using alternative sampling plans? .................................... 3

$\S 30.03$ What additional requirements do alternative sampling plans should meet? ................. 3

SECTION 40 - READY-TO-USE SAMPLING PLANS ................................................................... 4

Subsection 41 - General Directions ..................................................................................... 4

$\$ 41.01$ Under what conditions may I use ready-to-use sampling plans? ................................ 4

$\S 41.03$ If I am using a ready-to-use sampling plan this year, may I use it again for my next report year? .................................................................................................... 4

$\S 41.05$ For what modes are ready-to-use sampling plans available? ...................................... 4

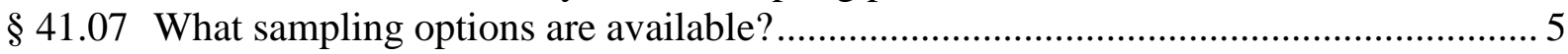

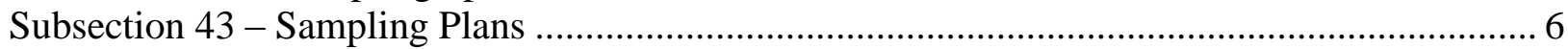

$\S 43.01$ What period-based sampling plans are available for non-scheduled services?............. 6

$\S 43.03$ What period-based ready-to-use sampling plans are available for bus services? ........ 6

$\S 43.05$ How does grouping of my bus routes affect sampling and estimation procedures? .... 7

$\$ 43.07$ What period-based sampling plans are available for commuter rail? ......................... 7

$\$ 43.09$ What period-based sampling plans are available for other rail modes? ........................ 8

$\S 43.11$ What interval-based ready-to-use sampling plans are available for bus services? ...... 8

SECTION 50 - TEMPLATE SAMPLING PLANS ........................................................................ 10

Subsection 51 - General Directions ...................................................................................... 10

§ 51.01 Under what conditions should I develop a template sampling plan for next year?.... 10

$\S 51.02$ Should I consider template sampling plans if I am a small system and want to sample? ..................................................................................................... 10

$\S 51.03$ Does this Manual have a companion tool that I may use? ....................................... 10

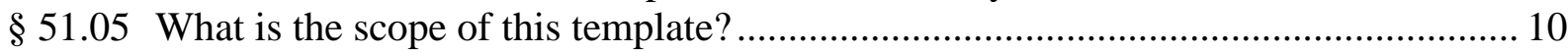

$\$ 51.07$ Do I need to have knowledge of statistics to use this template? ............................... 10

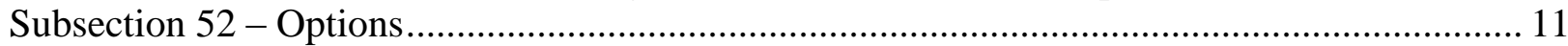

$\S 52.01$ What types of options does this template provide in its sampling plans? .................. 11

$\$ 52.03$ Must I pick a specific unit of sampling and measurement to use this template? ....... 11

$\$ 52.04$ What options does this template provide on sampling structure? .............................. 11

$\S 52.05$ What options does this template provide on sampling frequency? ............................ 11

$\$ 52.07$ What should I consider in choosing a sampling frequency? .................................... 12

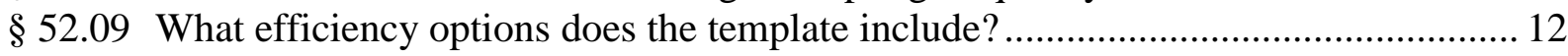

$\S 52.11$ Which of these efficiency options are available to me? ............................................ 13

$\S 52.13$ How do I divide my scheduled service if I want to consider the grouping option? ... 13

$\S 52.15$ How do I group my vanpool service if I serve commuters only? ............................. 14

$\S 52.17$ What should I consider in general when I consider grouping my service?................ 14

Subsection 53 - Input Data .......................................................................................... 14

§5.01 What data must I have to use this template? .......................................................... 14

$\S 53.03$ Must I identify and correct errors with my sample data before I enter them into the template? 
$\S 53.05$ Do interval-based sampling plans have any special requirement for the sample data used in developing them? ................................................................................... 15

$\S 53.07$ What other data do I need to use this template?...................................................... 15

$\S 53.09$ What sample data should I use to develop template sampling plans for next year? .. 16

$\S 53.11$ How does this template deal with fluctuations in sample data from one year to another for a given service? ................................................................................ 16

$\S 53.13$ Why do such annual fluctuations in sample data require a margin of safety? ........... 16

$\S 53.15$ How does this template ensure adequate sample size for developing template

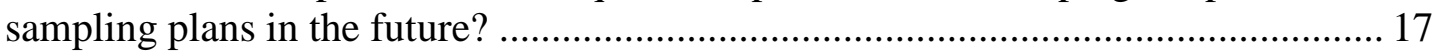

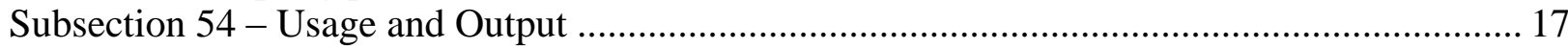

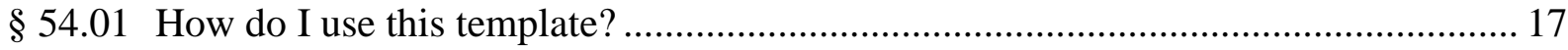

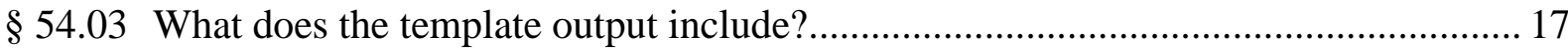

Subsection 55 - Selecting a Sampling Plan ........................................................................ 18

$\S 55.01$ What should I consider in selecting one from the set of template sampling plans?... 18

$\S 55.03$ Should I keep a copy of the used template that contains my sample data and my chosen template sampling plan?............................................................................. 18

$\S 55.05$ How does my choice of efficiency options affect my choice of estimation methods? 19

$\$ 55.07$ May I change template sampling plans from one report year to another? ................. 19

\$ 55.09 May I change sampling plans during a report year? ................................................... 19

$\S 55.11$ How should I implement a template sampling plan? ................................................ 19

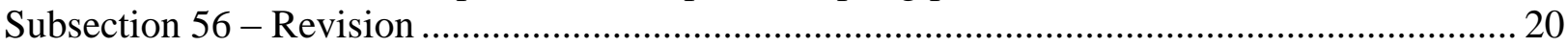

$\S 56.01$ When does this subsection apply to me? ............................................................... 20

$\S 56.03$ Why do I need to consider revision? .................................................................... 20

$\$ 56.05$ Under what conditions should I revise my template sampling plan? ......................... 20

$\$ 56.07$ How do I know if next year is a mandatory revising year for me? ........................... 20

$\S 56.09$ What are considered major changes to my service?................................................ 21

$\S 56.11$ How do I determine if I must revise my template sampling plan after I have made

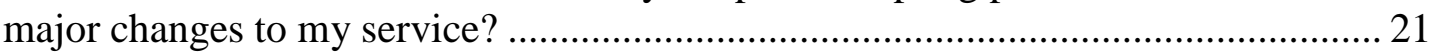

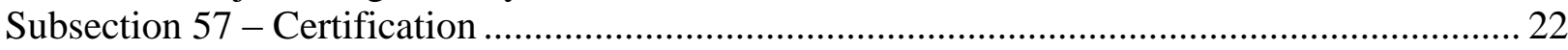

$\S 57.01$ How do I certify my template sampling plan? .................................................... 22

SECTION 60 - DATA COLLECTION ........................................................................................ 23

Subsection 61 - General Directions ..................................................................................... 23

$\S 61.01$ What are the basic elements of collecting sample data? .......................................... 23

$\S 61.03$ What criteria does this section cover to ensure that estimates of annual serviceconsumed data meet FTA's 95\% confidence and 10\% precision levels? ................... 23

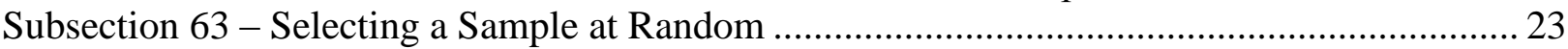

$\S 63.01$ What are the basic elements of selecting a sample at random?............................... 23

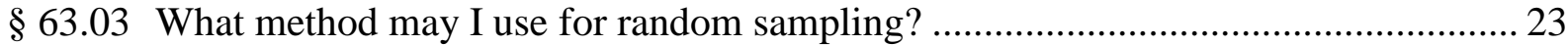

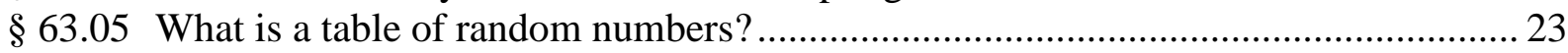

$\S 63.07$ What is the list of all service units that I expect to operate?....................................2 24

$\$ 63.09$ How do I develop the list of all service units for using a table of random numbers? 24

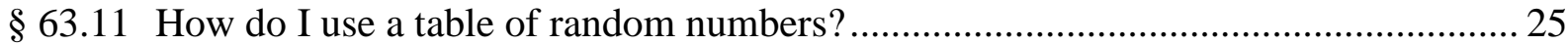

$\$ 63.13$ What are the pros and cons of using a table of random numbers? ............................ 26

$\S 63.15$ What information should I keep from my sampling process?...................................2 26

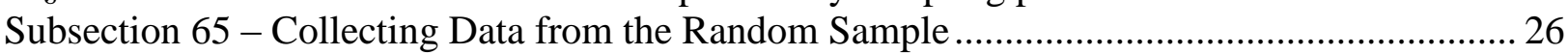

$\S 65.01$ What method may I use to collect the sample data? ..................................................2 26

$\S 65.03$ What approach may I use to determine PMT for each service unit of my sample?... 27 
$\S 65.05$ What data items must I collect to use the load-based approach for scheduled services?

$\S 65.07$ What data must I collect to use the load-based approach for non-scheduled

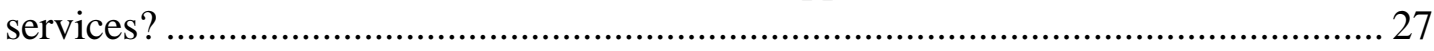

$\S 65.09$ How should I determine between-stop distances for the load-based approach? ....... 27

$\S 65.11$ What additional data should I collect to identify each service unit of my sample? ... 28

$\S 65.13$ When do I use the different approaches to determining PMT?............................. 28

$\S 65.15$ What instrument should I use to collect the data for the distance-based approach?.. 28

$\S 65.17$ What instrument should I use to collect data for the load-based approach? ............ 29

$\S 65.19$ What pre-survey procedures should I follow if I use a paper instrument and human ride checkers?

$\S 65.21$ What manual survey procedures should I follow for demand response (DR and DT) with the distance-based approach?

$\S 65.23$ What manual survey procedures should I follow for demand response (DR and DT) with the load-based approach?

$\S 65.25$ What manual survey procedures should I follow for commuter vanpool with the load-based approach?

$\S 65.27$ What instructions should I give my ride checkers if I do not use predetermined between-stop distances for fixed-route services?

$\S 65.29$ What steps should I take if I fail to collect the sample data from a particular unit?.. 32

Subsection 67 - Correcting Errors in the Raw Sample Data .............................................. 33

$\S 67.01$ What steps should I take after I have collected the sample data? .......................... 33

$\S 67.03$ What should I consider in designing the format for data recording? ....................... 33

$\S 67.05$ When should I enter my sample data?........................................................... 34

$\$ 67.07$ How should I process the entered data? ............................................................. 34

$\S 67.09$ How may I use the processed data to identify potential errors in the sample data?... 36

$\S 67.11$ How do I identify the sources of any errors? ................................................... 36

$\S 67.13$ How do I correct any errors? ............................................................................. 36

$\S 67.15$ What should I do if I fail to correct the data errors for a particular service unit?..... 38

$\S 67.17$ What steps should I take after I have identified and corrected errors in my data? .... 38

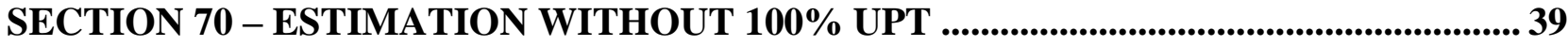

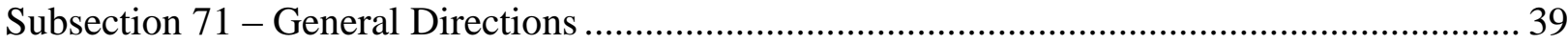

$\S 71.01$ What service-consumed data must I estimate without $100 \%$ UPT? ........................ 39

$\S 71.03$ What do I need to do in general to get estimates of these data items? ...................... 39

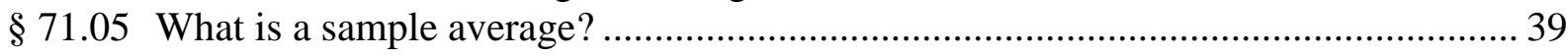

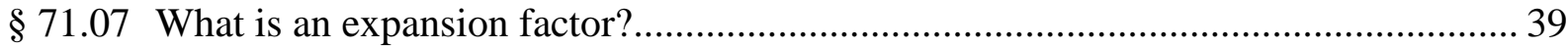

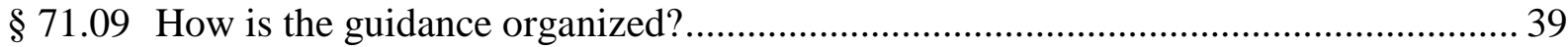

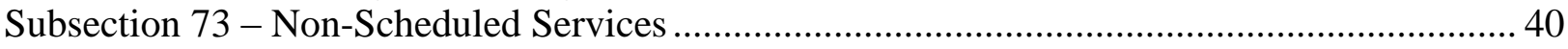

$\S 73.01$ What expansion factor should I use for non-scheduled service? ........................... 40

$\S 73.03$ What sample average should I use for non-scheduled service? .............................. 40

$\S 73.05$ How do I determine annual vehicle days actually operated for non-scheduled

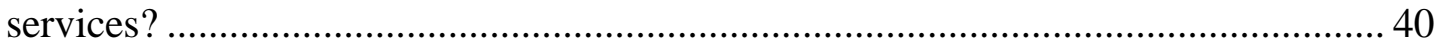

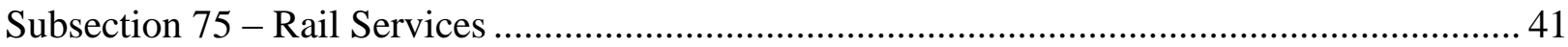

$\S 75.01$ What expansion factor should I use for rail services? ........................................ 41

$\S 75.03$ What sample average should I use for rail services?........................................ 41

$\S 75.05$ What steps should I follow to calculate sample averages? .................................. 42

$\S 75.07$ How should I determine annual services actually provided as expansion factors?.... 43 


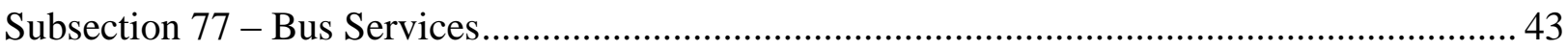

$\S 77.01$ What expansion factor should I use for bus services?............................................... 43

$\$ 77.03$ What sample average should I use for bus services? ................................................... 43

\$ 77.05 What steps should I follow to calculate sample averages? ....................................... 44

$\$ 77.07$ How should I determine annual services actually provided as expansion factors?.... 44

Subsection 79 - Estimating Service-Consumed Data .............................................................. 45

$\$ 79.01$ How should I estimate the annual total of my service-consumed data? .................... 45

$\S 79.03$ How should I estimate average daily of service-consumed data by type of service days?

$\S 79.05$ How should I estimate annual total UPT for each weekday period for commuter

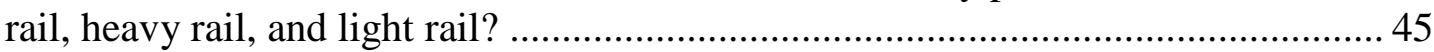

SECTION 80 - ESTIMATION WITH 100\% UPT ......................................................................... 46

Subsection 81 - General Directions ........................................................................................ 46

$\S 81.01$ What service-consumed data must I estimate with 100\% UPT? ............................... 46

$\S 81.03$ What do I need to do in general to get estimates of these data items? ........................ 46

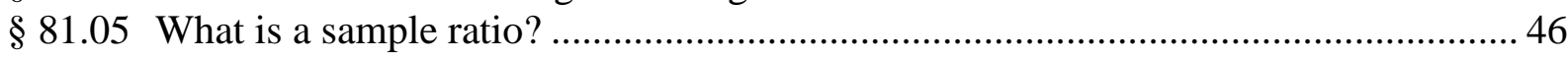

$\S 81.07$ What is an expansion factor when I have 100\% UPT? .......................................... 46

Subsection 83 - APTL Option ........................................................................................... 46

$\S 83.01$ What expansion factor should I use for the APTL option? ........................................4 46

$\$ 83.03$ What sample ratio should I use for the APTL option? ...............................................4 47

$\$ 83.05$ How should I determine the APTL from my sample? ................................................ 47

$\$ 83.07$ How should I estimate annual total PMT for the APTL option? ............................... 48

$\S 83.09$ How should I estimate average daily PMT by type of service days?......................... 49

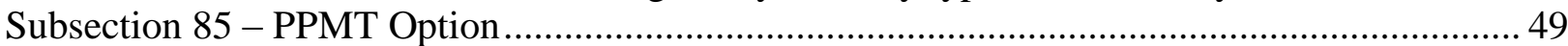

$\S 85.01$ What expansion factor should I use for the PPMT option? ...................................... 49

$\S 85.03$ How do I determine annual total PPMT? .................................................................. 49

$\S 85.05$ How should I get the sample total of PPMT for each service unit? ...........................5 50

$\S 85.07$ What sample ratio should I use for the PPMT option? ..............................................51

$\S 85.09$ How should I determine the PMT/PPMT ratio for a sample?.................................... 51

$\S 85.11$ How should I estimate annual total PMT for the PPMT option? ............................... 51

$\S 85.13$ How should I estimate average daily PMT by type of service days?......................... 51

SECTION 90 - APPENDIXES ...................................................................................................5 52

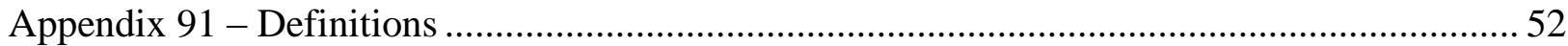

§ 91.01 What do the sampling-related terms mean? .........................................................52

$\S 91.03$ What do the data-collection terms mean? ............................................................... 54

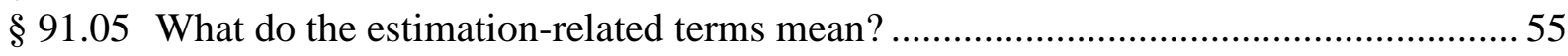

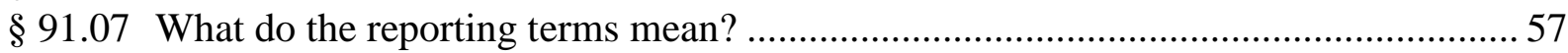

$\$ 91.09$ What abbreviations for general terms are used in this Manual? ................................ 57

$\S 91.11$ What abbreviations for NTD modes are used in this Manual? ..................................5 57

Appendix 92 - Distance-Based Survey Sheet............................................................................ 60

Appendix 93 - Load-Based Survey Sheet for Demand Response (DR or DT) ............................6 62

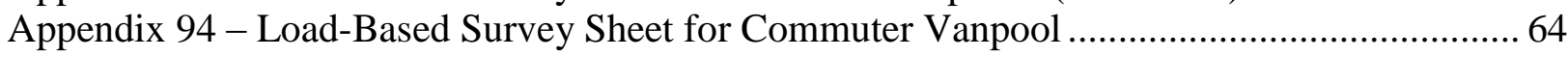

Appendix 95 - Load-Based Survey Sheet for Fixed-Route Service ................................................ 66

Appendix 96 - Daily Log Sheet for Commuter Vanpool ............................................................6 68

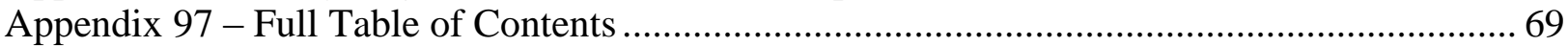

Appendix 98 - Table of Random Numbers ............................................................................... 73 


\section{Appendix 98 - Table of Random Numbers}




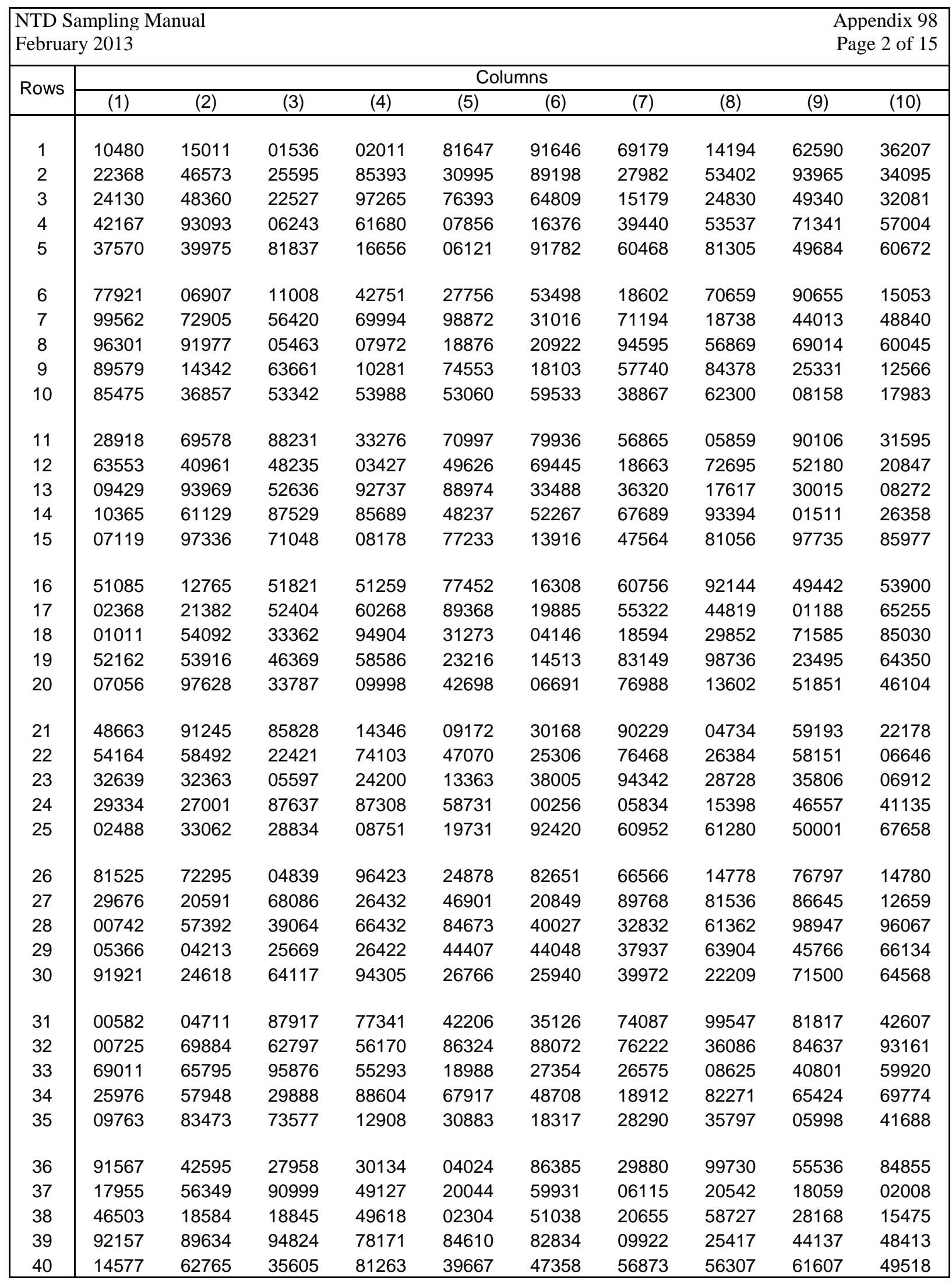




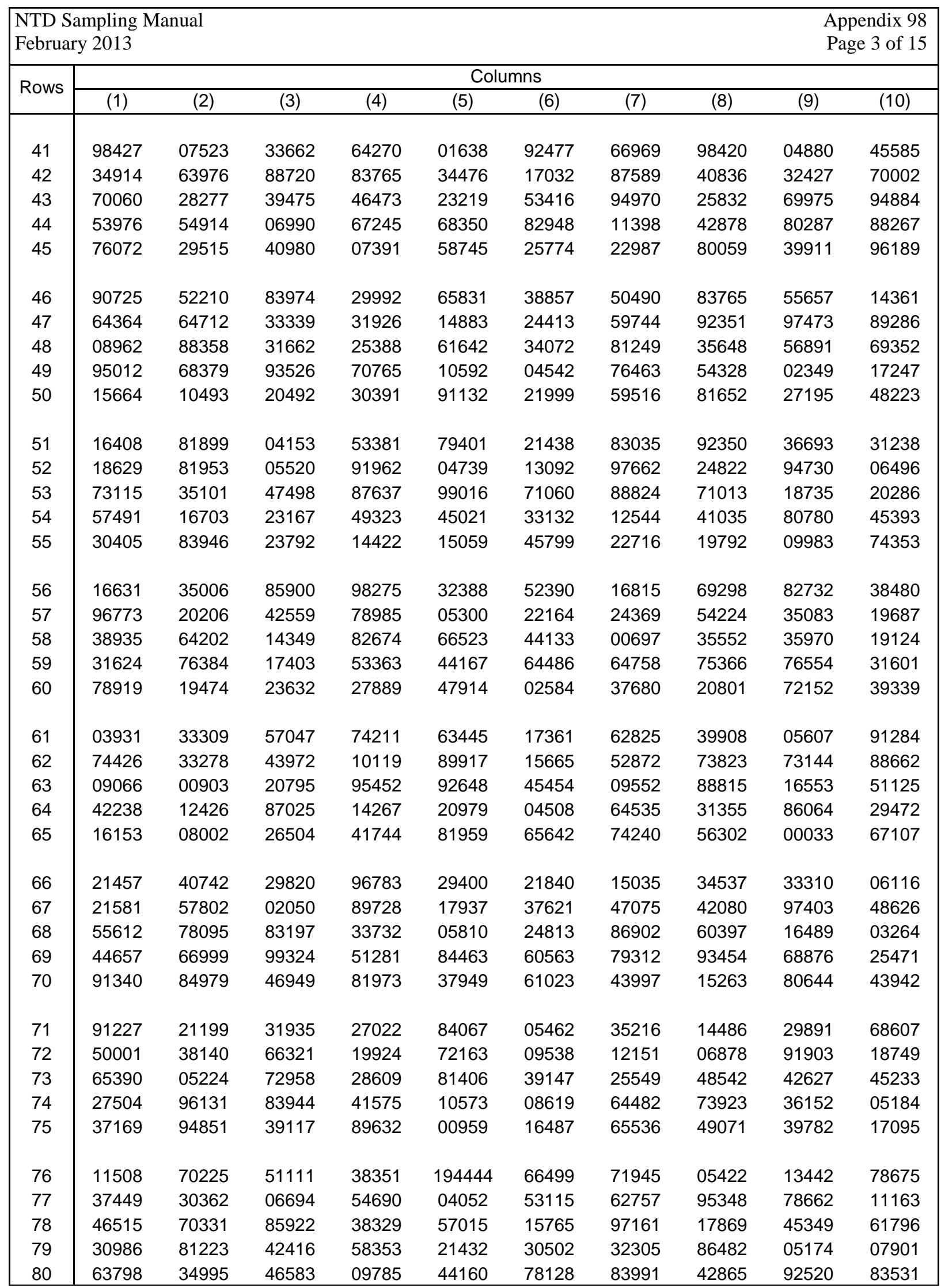




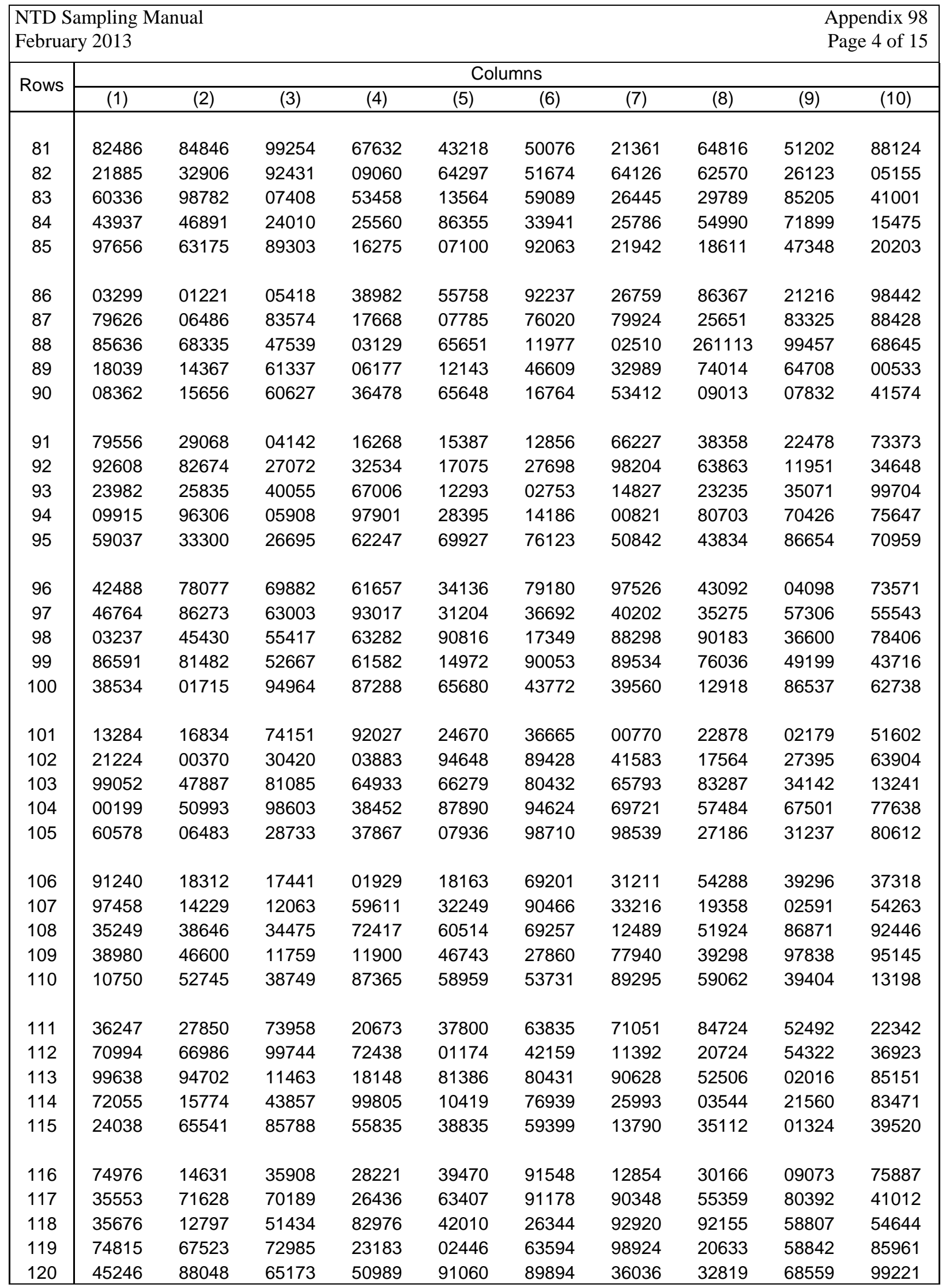




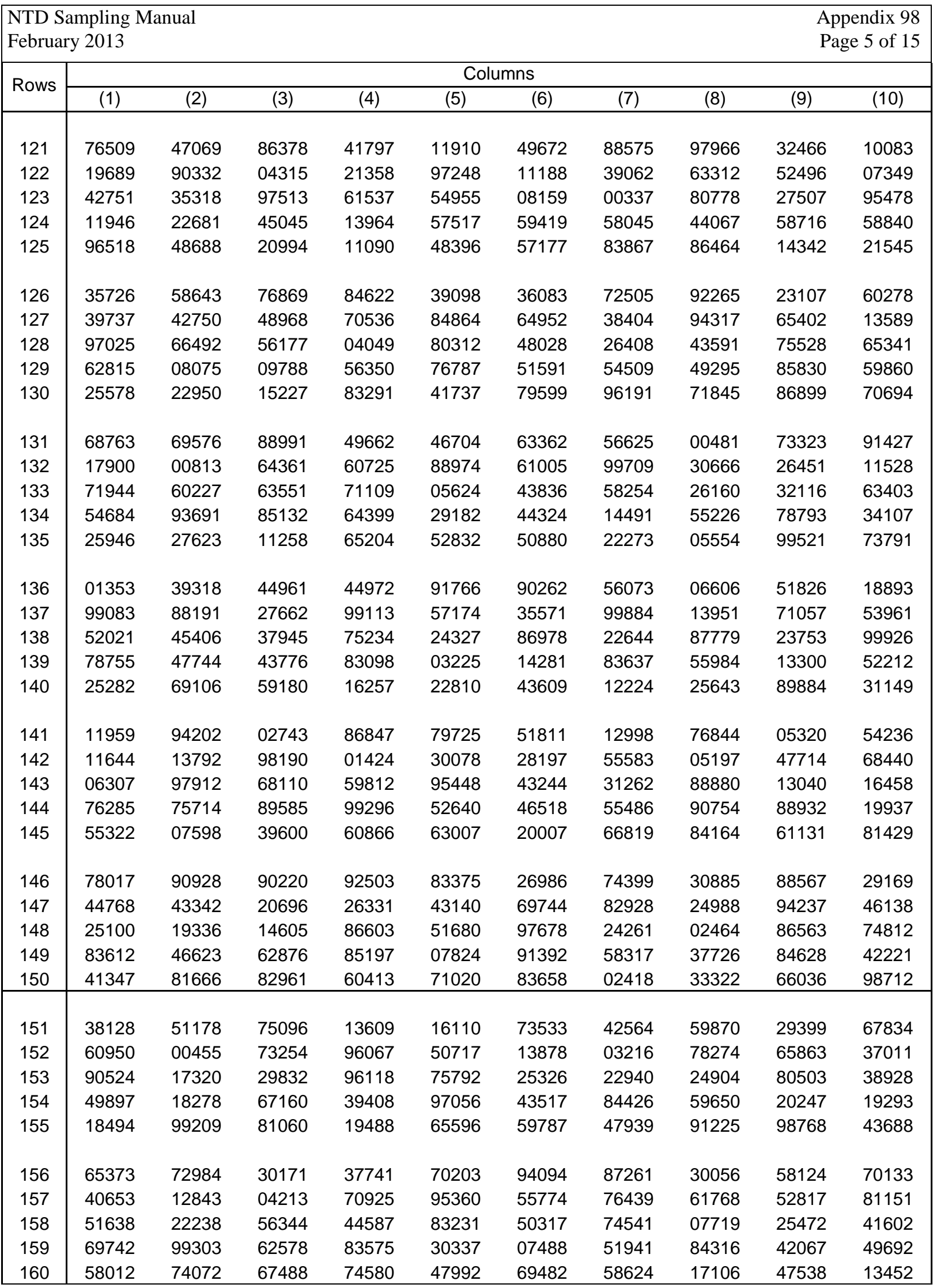




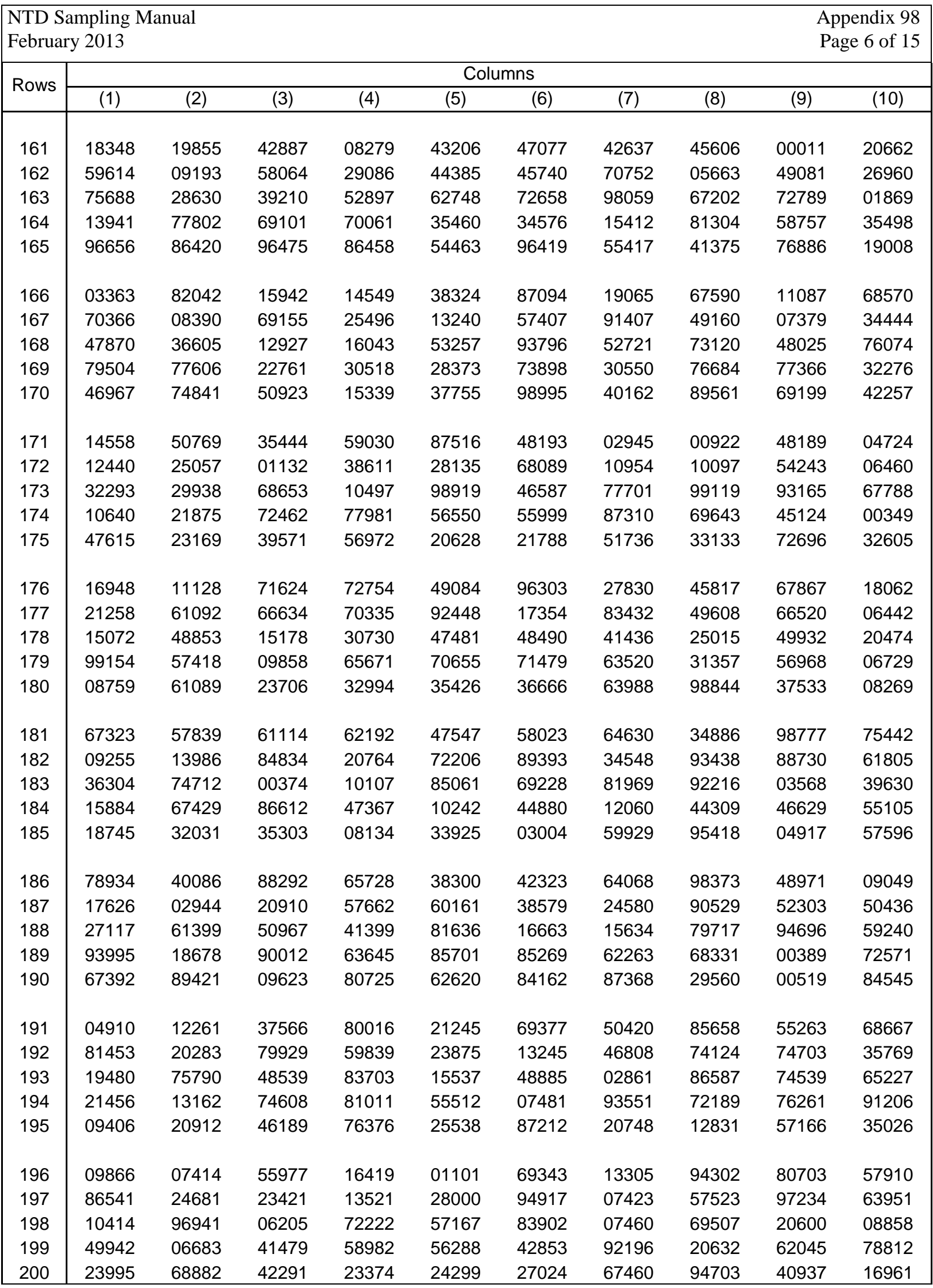




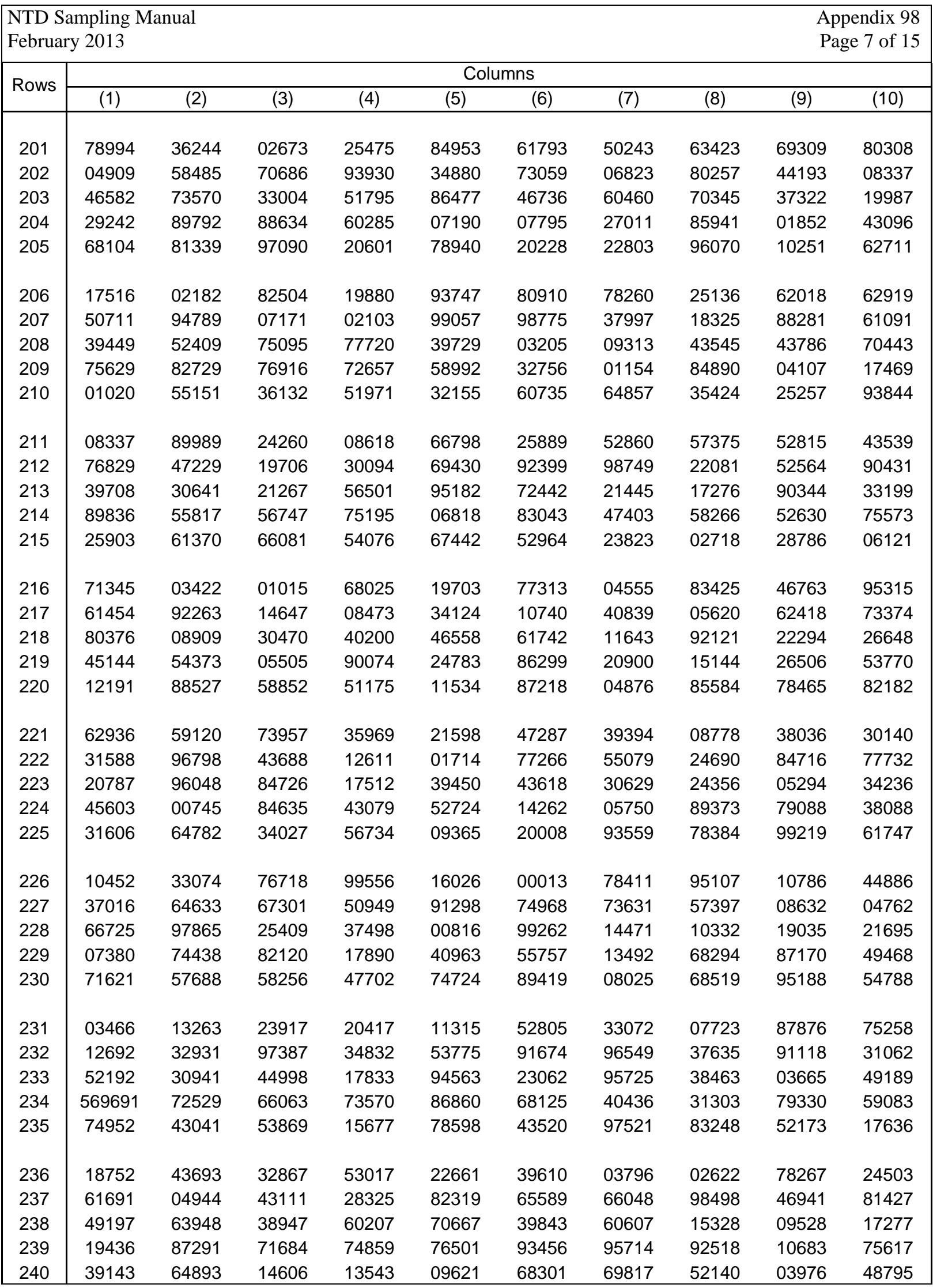




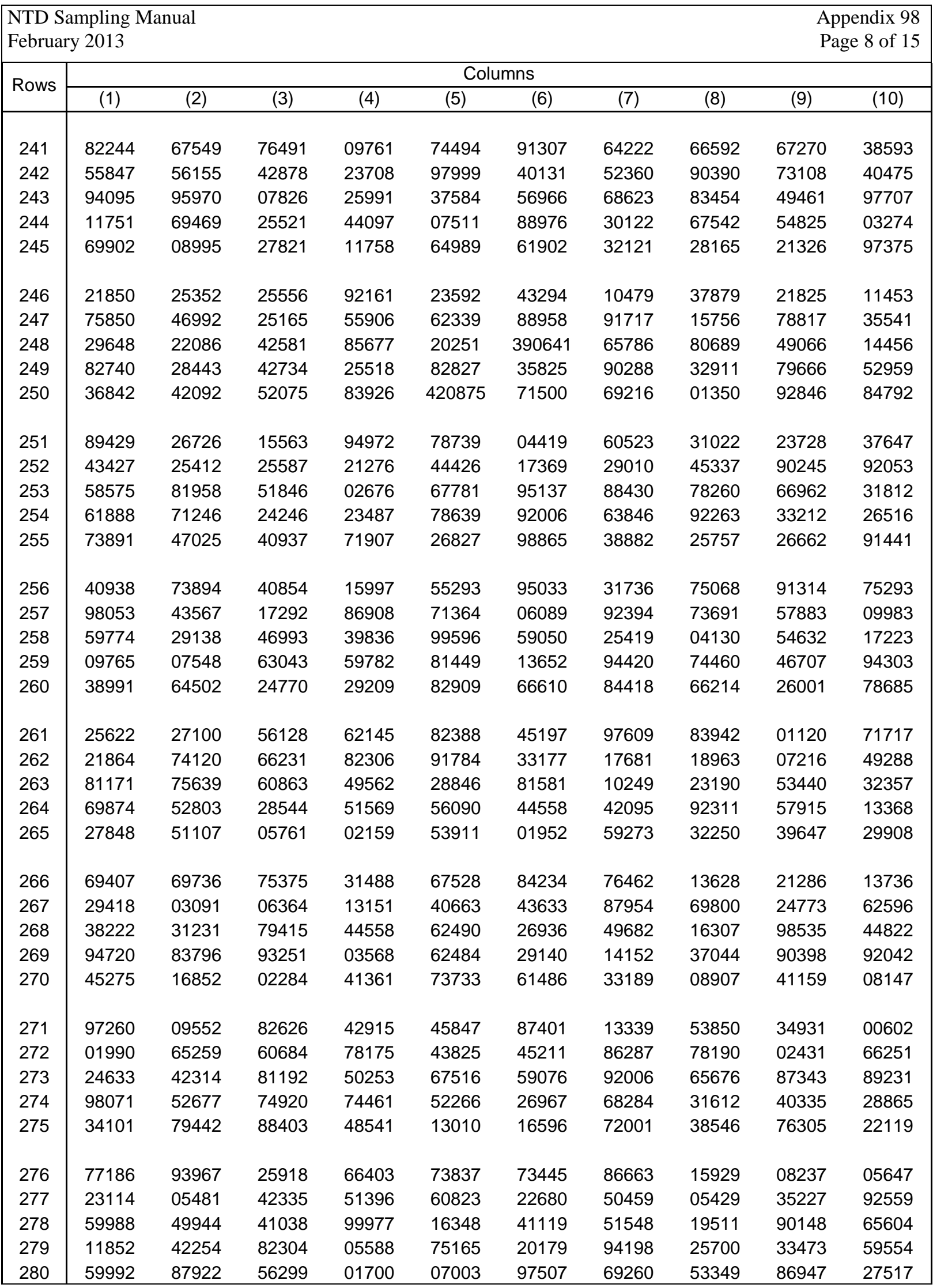




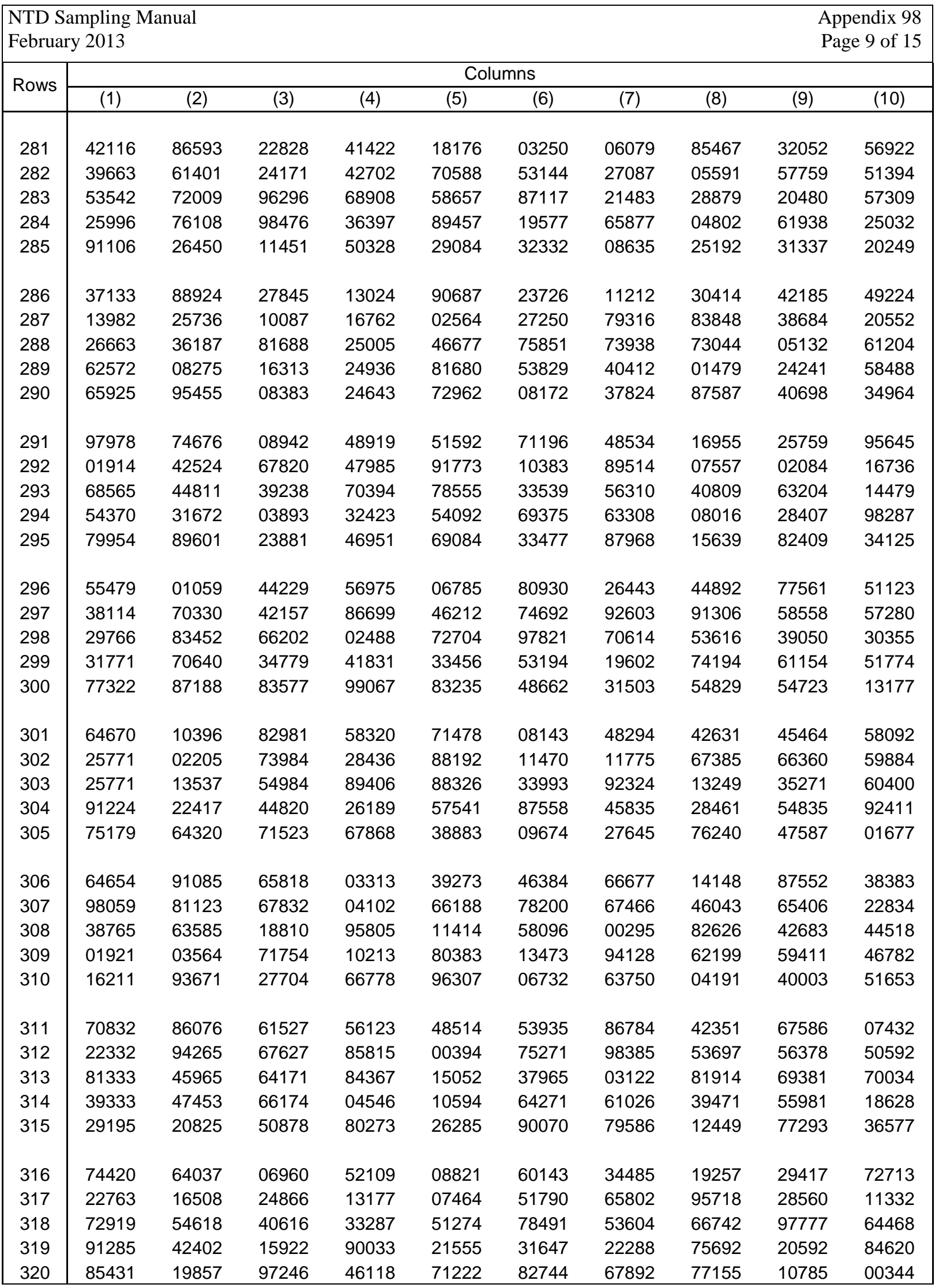




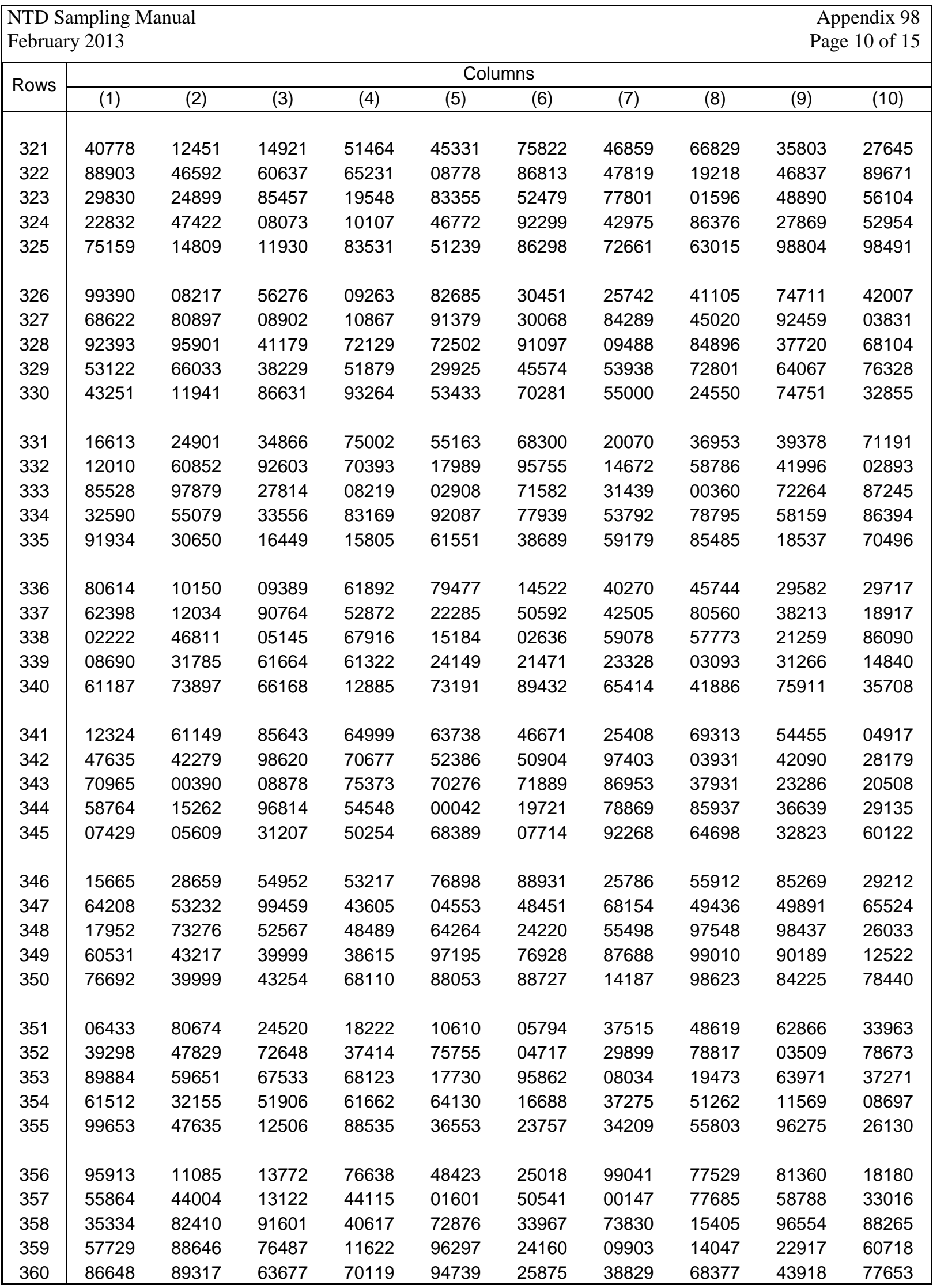




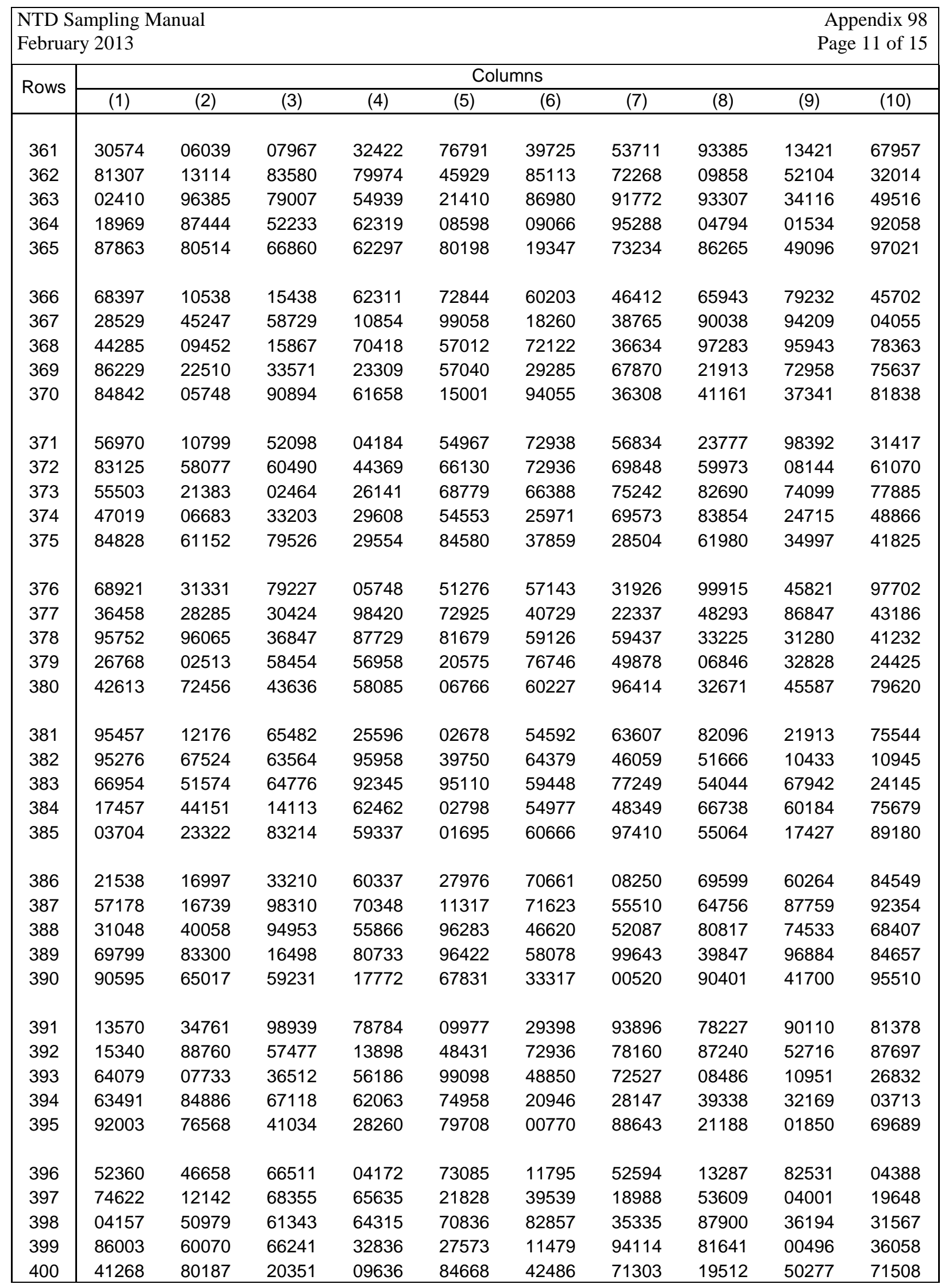




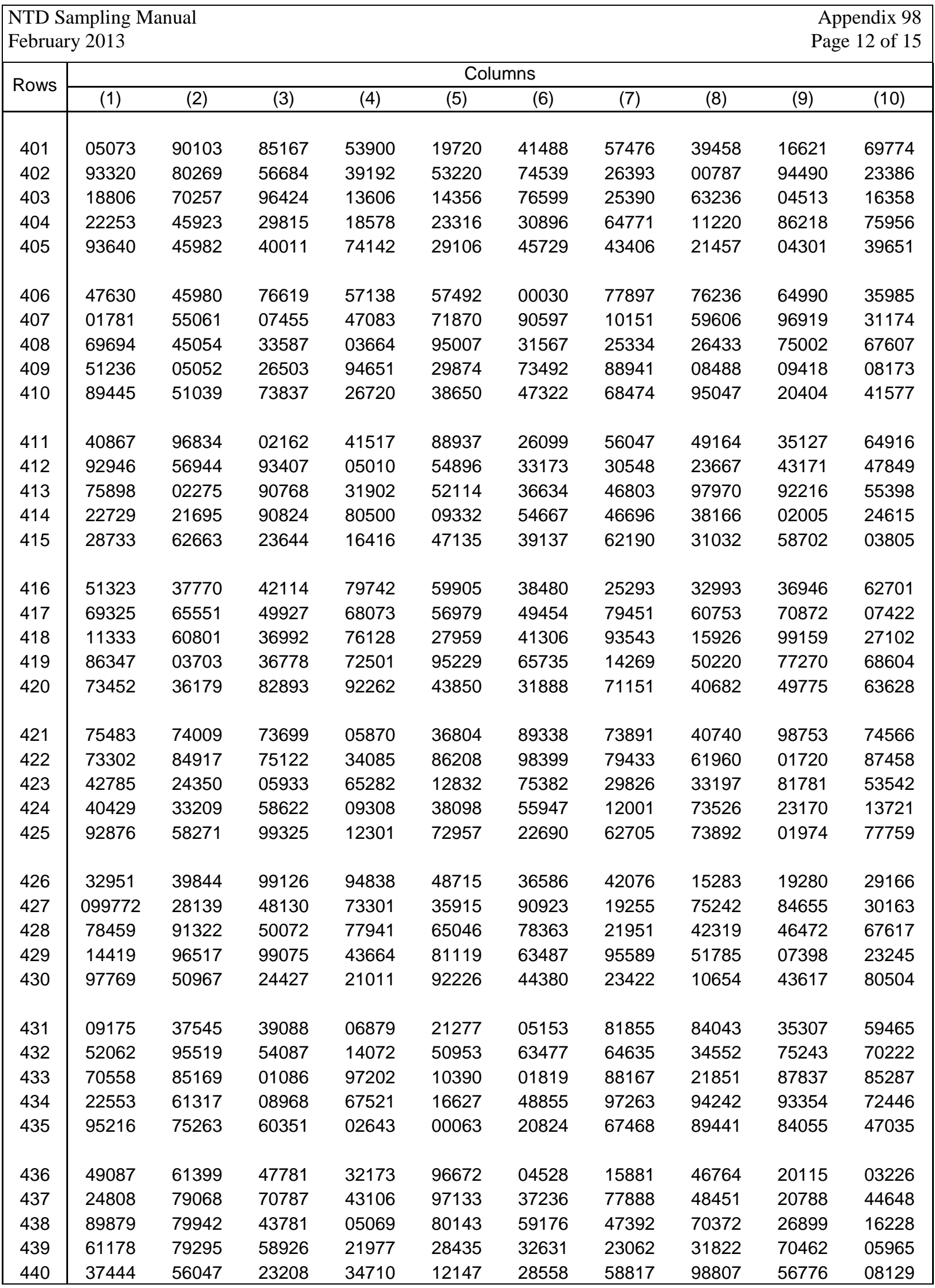




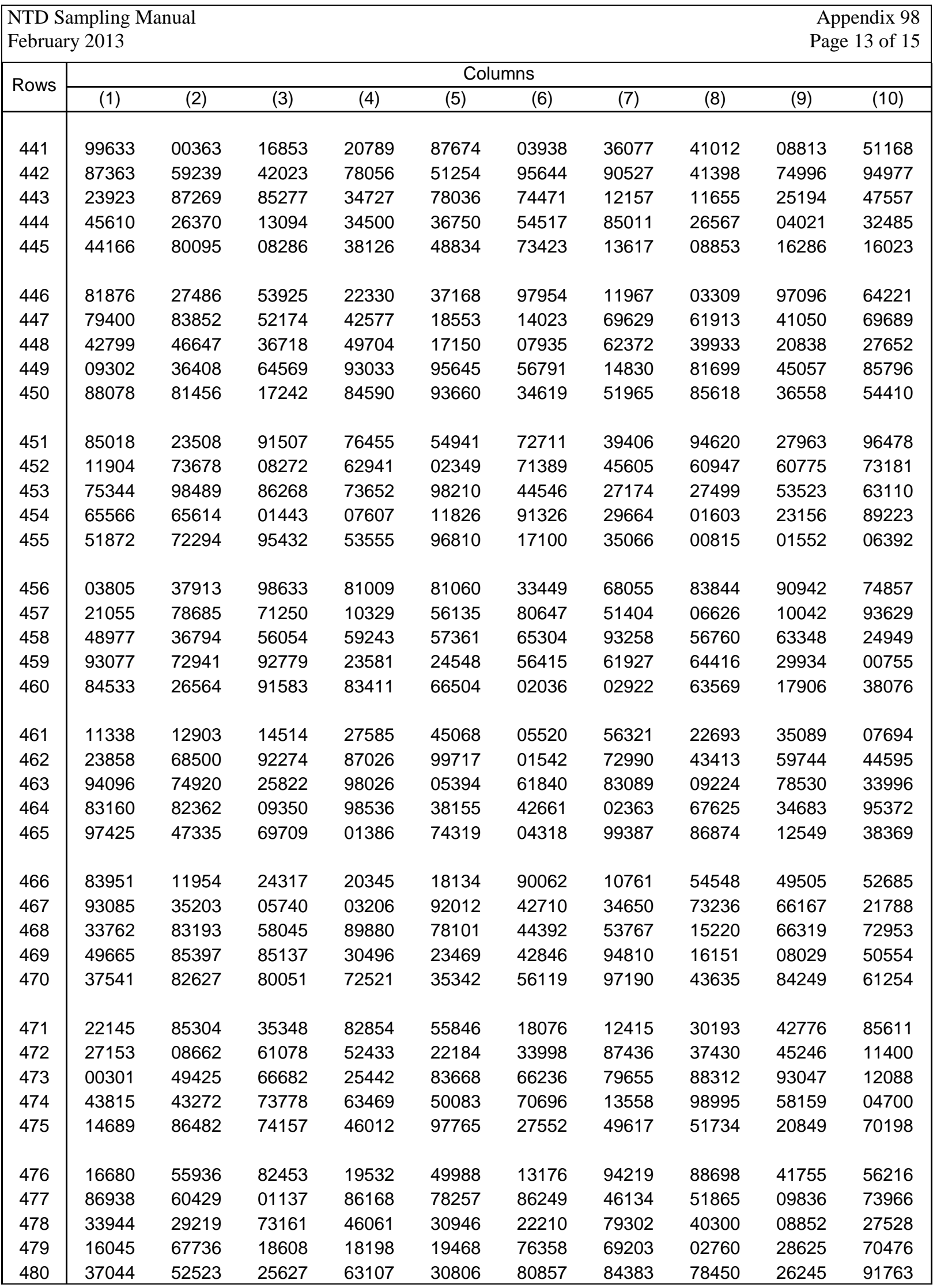




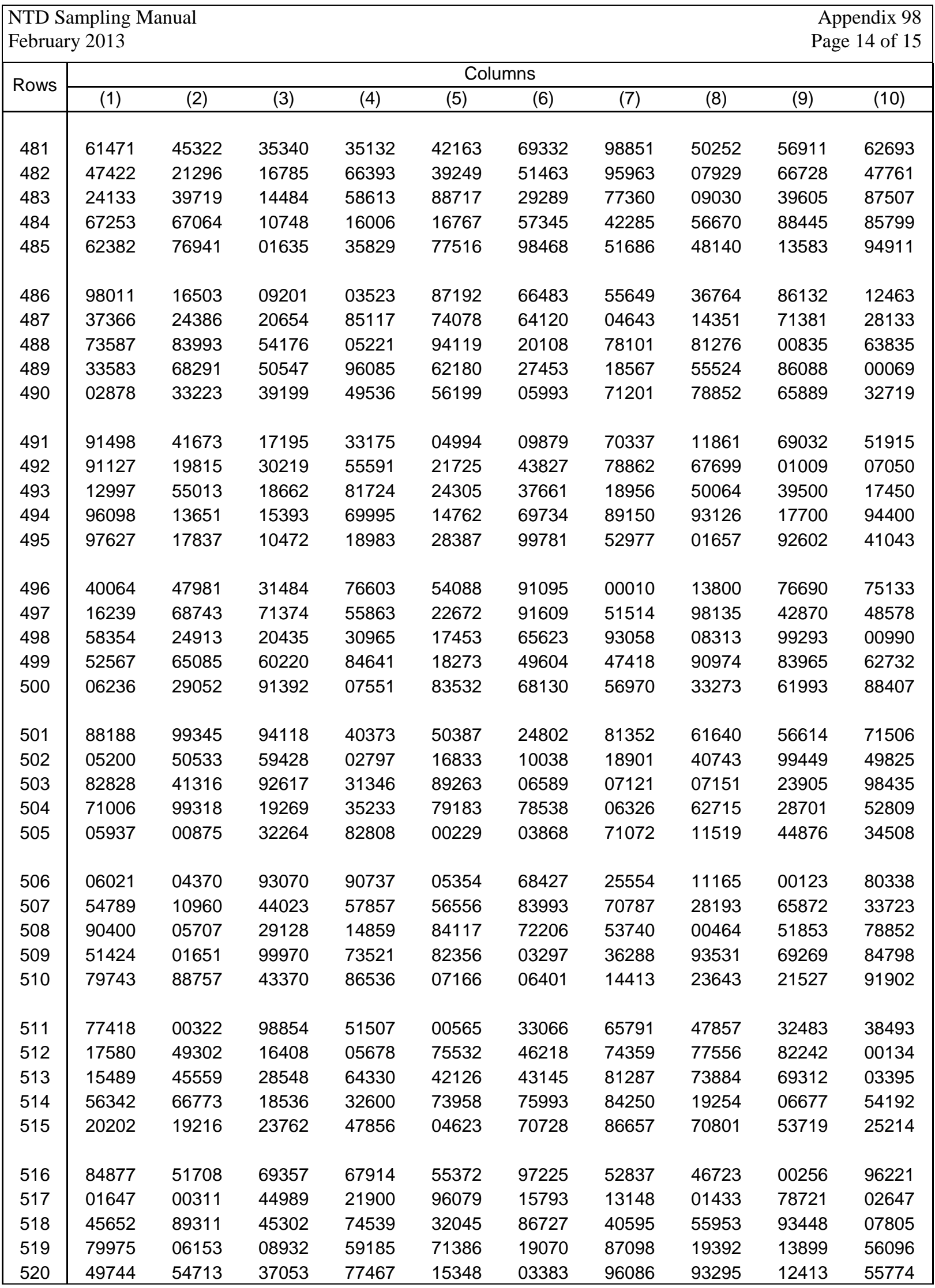




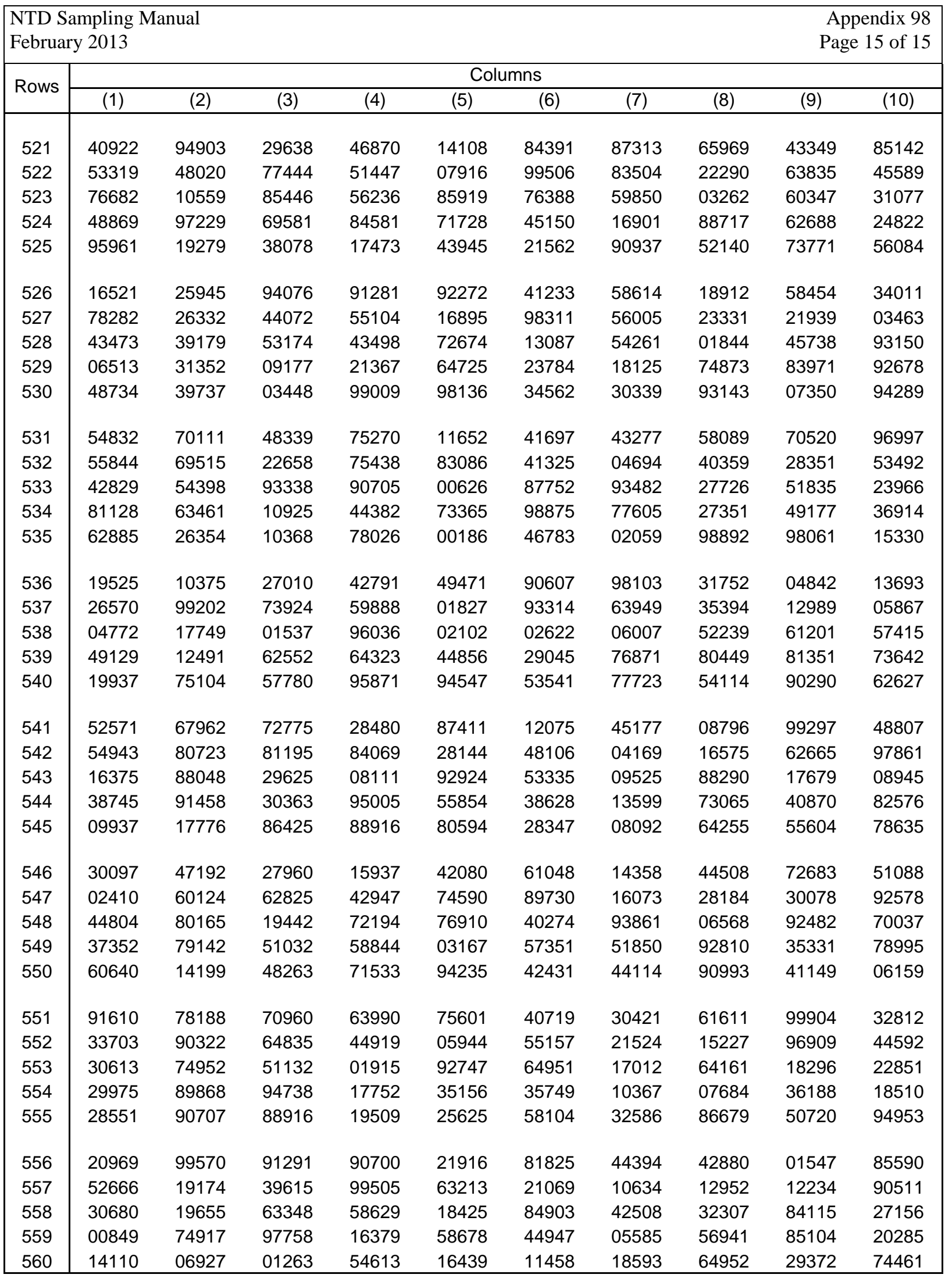

